\title{
Skeletal Diversity via Ring Contraction of Glycal-Derived Scaffolds
}

\author{
Adam R. Yeager, Geanna K. Min, John A. Porco, Jr., ${ }^{*}$ and Scott E. Schaus. ${ }^{*}$ \\ Department of Chemistry and Center for Chemical Methodology and Library Development (CMLD-BU), Boston \\ University, 24 Cummington St., Boston, Massachusetts 02215
}

\section{Supporting Information}

General Information: All reactions were carried out in oven or flame-dried glassware under an atmosphere of argon unless otherwise noted. ${ }^{1} \mathrm{H}$ NMR spectra were recorded on a Varian Mercury $400 \mathrm{MHz}$ spectrometer at ambient temperature and are reported in ppm relative to solvent $\left(\mathrm{CHCl}_{3}\right.$ at $7.26 \mathrm{ppm}$ ). Proton decoupled ${ }^{13} \mathrm{C}$ NMR spectra were recorded at $100.0 \mathrm{MHz}$ at ambient temperature, and are reported in ppm relative to solvent $\left(\mathrm{CHCl}_{3}\right.$ at $\left.77.0 \mathrm{ppm}\right)$. Data for ${ }^{1} \mathrm{H} \mathrm{NMR}$ are reported as follows: chemical shift, integration, multiplicity ( $a p p=$ apparent, $\mathrm{s}=$ singlet, $\mathrm{d}=$ doublet, $\mathrm{t}=$ triplet, $\mathrm{q}$ $=$ quartet, $\mathrm{m}=$ multiplet) and coupling constants (reported in Hz.). High pressure liquid chromatography/mass spectral (LC-MS) analyses were performed using a Micromass 2Q 2000 mass spectrometer in atmospheric pressure chemical ionization (APCI) or electrospray ionization (ESI) modes. LC separations were performed using a Waters Alliance 2996 module and a Waters Symmetry C8 column $(4.6 \times 30 \mathrm{~mm})$. ELS detection was performed using a Sedere Sedex 75 ELS detector. Product purities are reported as ELSD area percent. Infrared spectra were recorded on a Nicolet Nexus 670 FT-IR spectrophotometer. Low and high-resolution mass spectra were obtained in the Boston University Mass Spectrometry Laboratory using Finnegan MAT-90 and Waters Q-Tof spectrometers. Microwave mediated reactions were performed using the CEM Discover / Explorer system, equipped with either $10 \mathrm{~mL}$ or $80 \mathrm{~mL}$ reaction vessels. Chromatography was performed using the ISCO Companion system. Reaction planning and was performed using the Synthematix electronic notebook program (http://www.synthematix.com/). X-ray crystal structures were obtained by Dr. Emil Lobkovsky (Department of Chemistry and Chemical Biology, Cornell University). Analytical thin layer chromatography was performed on $0.25 \mathrm{~mm} \mathrm{SiO}_{2} 60-\mathrm{F}$ plates. Flash chromatography was performed using 200-400 mesh $\mathrm{SiO}_{2}$ (Scientific Absorbent Incorporated). Methylene chloride and tetrahydrofuran were purified by passing through two packed columns of neutral alumina (Glass Contour, Irvine, $\mathrm{CA}$ ). $\mathrm{MeOH}$, dichloroethane, and dimethyformamide were used as supplied from Dri-Solv (EMD) bottles. MP-Carbonate ( $2.98 \mathrm{mmol} / \mathrm{gram})$ was obtained from Biotage. All other reagents and solvents were used as supplied by Sigma-Aldrich, Fluka, Acros, and Strem Chemicals. 


\section{Synthesis of $C$-Glycosides 3c (cf. Scheme 2):}
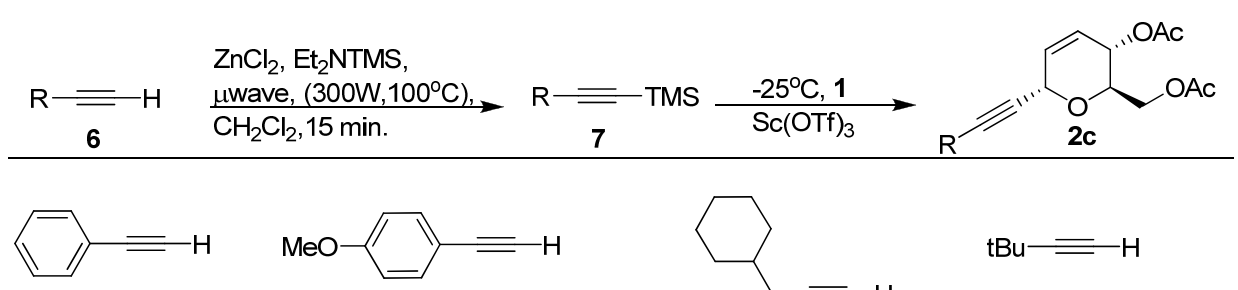

SI-1 (88\%),

21:1 $\alpha: \beta$

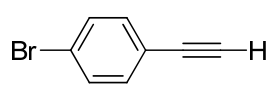

SI-2 (88\%)

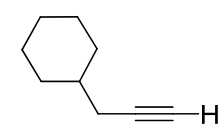

$\mathrm{tBu}=\mathrm{H}$

SI-3 (73\%)

SI-4 (74\%)
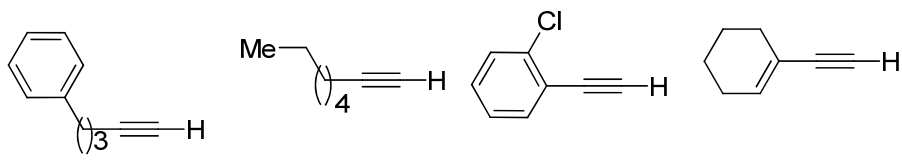

SI-5 (60\%)

SI-6 (72\%)

SI-7(77\%)

SI-8 (35\%)

SI-9 (55\%)
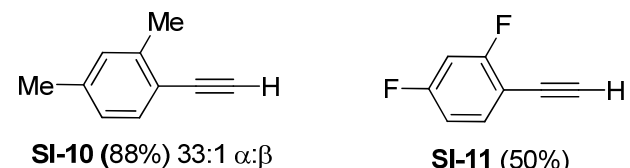

SI-11 (50\%)

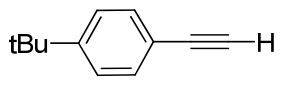

SI-12 (51\%)

Table SI-1: Alkyne addition to glycals

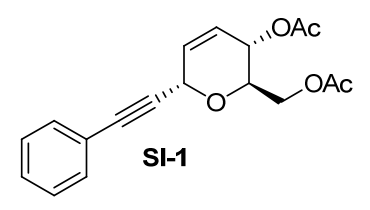

Alkynyl $\boldsymbol{C}$-glycoside SI-1: Zinc chloride $(520 \mathrm{mg} 3.8 \mathrm{mmol})$, was added to a flame-dried $80 \mathrm{~mL}$-microwave flask under an inert atmosphere (glove box). The flask was removed from the glove box and placed under argon. Dichloroethane $(10 \mathrm{~mL})$ was added, followed by $N, N$ - diethyl- 1,1,1trimethylsilylamine $(0.570 \mathrm{~mL}, 2.9 \mathrm{mmol})$ and $p$-methoxyphenylacetylene

$(0.250 \mathrm{~mL}, 1.9 \mathrm{mmol})$. The flask was sealed and heated at $150^{\circ} \mathrm{C}$ using microwave irradiation (150-300 W, Powermax enabled) for $15 \mathrm{~min}$. The reaction was again placed under argon and transferred to a $-25^{\circ} \mathrm{C}$ cold bath. Tri-O-acetyl-D-glucal $(260 \mathrm{mg}, 0.96 \mathrm{mmol})$ and scandium triflate $\left(20 \mathrm{mg}, 0.05 \mathrm{mmol}\right.$ ) were added. After $1.5 \mathrm{~h}$, the reaction was diluted with sat. $\mathrm{NaHCO}_{3}$ and extracted with $\mathrm{CH}_{2} \mathrm{Cl}_{2}$. The organic fraction was washed with sat. brine, dried, and concentrated. Chromatography over $\mathrm{SiO}_{2}(0-40 \%$ EtOAc / pet. ether) provided SI-1 $(0.528 \mathrm{~g}, 88 \%)$ as a viscous oil. Spectral data were in agreement with reported literature values. ${ }^{1}$

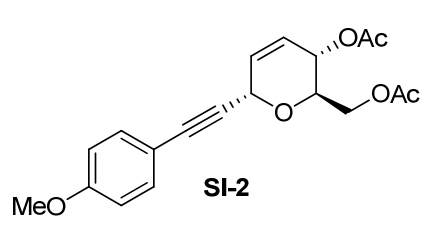

Alkynyl $\boldsymbol{C}$-glycoside SI-2: Zinc chloride (520 mg $3.8 \mathrm{mmol}$ ), was added to a flame-dried $80 \mathrm{~mL}$-microwave flask under an inert atmosphere (glove box). The flask was removed from the glove box and placed under argon. Dichloroethane $(10 \mathrm{~mL})$ was added, followed by $N, N$ - diethyl-1,1,1trimethylsilylamine $(0.570 \mathrm{~mL}, 2.9 \mathrm{mmol})$ and $p$-methoxyphenylacetylene $(0.250 \mathrm{~mL}, 1.9 \mathrm{mmol})$. The flask was sealed and heated using microwave irradiation $\left(150^{\circ} \mathrm{C}\right.$, 150-300 W, Powermax enabled) for $15 \mathrm{~min}$. The reaction was again placed under argon and transferred to a $-25^{\circ} \mathrm{C}$ cold bath. Tri-O-acetyl-D-glucal $(260 \mathrm{mg}, 0.96 \mathrm{mmol})$ and scandium triflate

1 J.S Yaday, B.V.S. Reddy, C.V. Rao, M.S. Reddy, Synthesis 2003, 2, 247-250 
(20 mg, $0.05 \mathrm{mmol}$ ) were added. After $1.5 \mathrm{~h}$ the reaction was diluted with sat. $\mathrm{NaHCO}_{3}$ and extracted with $\mathrm{CH}_{2} \mathrm{Cl}_{2}$. The organic fraction was washed with sat. brine, dried, and concentrated. Chromatography over $\mathrm{SiO}_{2}(0-40 \%$ EtOAc / pet. ether) provided SI-2 (292 mg, 88\%) as a viscous oil. ${ }^{1} \mathrm{H}$ NMR (400 MHz, $\left.\mathrm{CDCl}_{3}\right): \delta 7.38(\mathrm{~d}, 2 \mathrm{H}, J=13.2), 6.84$ (d, 2H, $\left.J=11.2\right), 5.97(\mathrm{~d}, 1 \mathrm{H}, J=10.0), 5.97$ (d, 1H, $J=9.6), 5.34$ (d, 1H, $J=8.8), 5.18$ (brs, $1 \mathrm{H}), 4.26$ (d, 2H, $J=3.2), 4.17(\mathrm{~m}, 1 \mathrm{H}), 3.84(\mathrm{~s}, 3 \mathrm{H})$, 2.09 (s, 3H), 2.08 (s, 3H) ppm; ${ }^{13} \mathrm{C}$ NMR (100 MHz, $\left.\mathrm{CDCl}_{3}\right): \delta 170.9,170.3,159.9,133.3,130.2$, 129.4, 125.2, 113.9, 86.6, 83.2, 69.8, 64.8, 64.5, 63.0, 55.3, 21.0, 20.8 ppm; IR (neat): 3743, 2955, $2831,2217,1740,1654,1608,1507,1231,1041 \mathrm{~cm}^{-1} ;[\alpha]_{\mathrm{D}}^{23}=-80^{\circ}\left(\mathrm{c}=1.0 \mathrm{CHCl}_{3}\right)$.

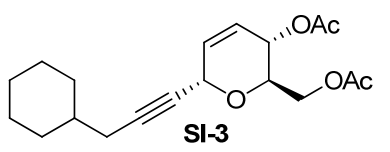

Alkynyl $\boldsymbol{C}$-glycoside SI-3: Zinc chloride (800 mg $5.9 \mathrm{mmol}$ ), was added to a flame-dried $80 \mathrm{~mL}$-microwave flask under an inert atmosphere (glove box). The flask was removed from the glove box and placed under argon. Dichloroethane $(10 \mathrm{~mL})$ was added, followed by $N, N$-diethyl-1,1,1-trimethylsilylamine $(0.800 \mathrm{~mL}$, $4.0 \mathrm{mmol})$ and 3-cyclohexyl-1-propyne $(0.530 \mathrm{~mL}, 3.7 \mathrm{mmol})$. The flask was sealed and heated using microwave irradiation $\left(150^{\circ} \mathrm{C}, 150-300 \mathrm{~W}\right.$, Powermax enabled) for $15 \mathrm{~min}$. The reaction was again placed under argon and transferred to a $-25^{\circ} \mathrm{C}$ cold bath. Tri-O-acetyl-D-glucal $(500 \mathrm{mg}, 1.8$ $\mathrm{mmol}$ ) and scandium triflate $(20 \mathrm{mg}, 0.4 \mathrm{mmol})$ were added. After $1.5 \mathrm{~h}$, the reaction was diluted with sat. $\mathrm{NaHCO}_{3}$ and extracted with $\mathrm{CH}_{2} \mathrm{Cl}_{2}$. The organic fraction was washed with sat. brine, dried, and concentrated. Chromatography over $\mathrm{SiO}_{2}(0-40 \%$ EtOAc / pet. ether) provided SI-3 (451 $\mathrm{mg}, 73 \%$ ) as a viscous oil. ${ }^{1} \mathrm{H}$ NMR $\left(400 \mathrm{MHz}, \mathrm{CDCl}_{3}\right): \delta 5.90(\mathrm{ddd}, 1 \mathrm{H}, J=1.6,2.8,10.3), 5.75$ $(\mathrm{dd}, 1 \mathrm{H}, J=2.0,10.4), 5.28(\mathrm{dd}, 1 \mathrm{H}, J=2.0,10.0), 4.97(\mathrm{~m}, 1 \mathrm{H}), 4.22(\mathrm{~m}, 2 \mathrm{H}), 4.11(\mathrm{~m}, 1 \mathrm{H}), 2.10(\mathrm{~m}$ $8 \mathrm{H}), 1.73(\mathrm{~m}, 5 \mathrm{H}), 1.46(\mathrm{~m}, 1 \mathrm{H}), 0.97-1.23(\mathrm{~m}, 5 \mathrm{H}) \mathrm{ppm} ;{ }^{13} \mathrm{C}$ NMR $\left(100 \mathrm{MHz}, \mathrm{CDCl}_{3}\right): \delta 170.8$, $170.2,129.6,125.0,119.8,88.5,81.8,69.7,64.8,64.4,63.0,28.9,25.6,22.1,21.3,21.0,20.8$ ppm; IR (neat): $3382,2939,2862,1732,1650,1375,1242 \mathrm{~cm}^{-1} ;[\alpha]_{\mathrm{D}}^{23}=-52^{\circ}\left(\mathrm{c}=1.0 \mathrm{CH}_{2} \mathrm{Cl}_{2}\right)$.

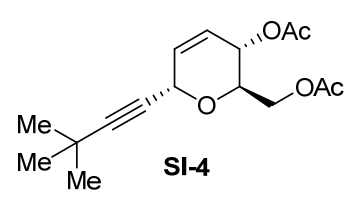

Alkynyl $\boldsymbol{C}$-glycoside SI-4: Zinc chloride (800 $\mathrm{mg} 5.9 \mathrm{mmol}$ ), was added to a flame-dried $80 \mathrm{~mL}-m i c r o w a v e ~ f l a s k ~ u n d e r$ an inert atmosphere (glove box). The flask was removed from the glove box and placed under argon. Dichloroethane $(10 \mathrm{~mL})$ was added, followed by $N, N-$ diethyl-1,1,1trimethylsilylamine $(0.800 \mathrm{~mL}, 4.0 \mathrm{mmol})$ and 3,3-dimethyl-1-butyne (300 $\mathrm{mg}, 3.7 \mathrm{mmol})$. The flask was sealed and heated using microwave irradiation $\left(150^{\circ} \mathrm{C}, 150-300 \mathrm{~W}\right.$, Powermax enabled) for 15 min. The reaction was again placed under argon and transferred to a $-25^{\circ} \mathrm{C}$ cold bath. Tri-O-acetyl-D-glucal $(500 \mathrm{mg}, 1.8 \mathrm{mmol})$ and scandium triflate $(140 \mathrm{mg}, 0.2 \mathrm{mmol})$ were added. After $1.5 \mathrm{~h}$, the reaction was diluted with sat. $\mathrm{NaHCO}_{3}$ and extracted with $\mathrm{CH}_{2} \mathrm{Cl}_{2}$. The organic fraction was washed with sat. brine, dried, and concentrated. Chromatography over $\mathrm{SiO}_{2}(0-40 \%$ EtOAc / pet. ether) provided SI-4 (0.305 g, 60\%) as a viscous oil. ${ }^{1} \mathrm{H}$ NMR $\left(400 \mathrm{MHz}, \mathrm{CDCl}_{3}\right): \delta 5.84$ (ddd, 1H, $J=1.8,3.6,10.2), 5.67(\mathrm{dt}, 1 \mathrm{H}, J=2.0,10.0), 5.23(\mathrm{~m}, 1 \mathrm{H}), 4.91(\mathrm{~m}, 1 \mathrm{H}), 4.10(\mathrm{~m}, 1 \mathrm{H}), 2.06$ $(\mathrm{s}, 3 \mathrm{H}), 2.05(\mathrm{~s}, 3 \mathrm{H}), 1.18(\mathrm{~s}, 9 \mathrm{H}) \mathrm{ppm} ;{ }^{13} \mathrm{C} \mathrm{NMR}\left(100 \mathrm{MHz}, \mathrm{CDCl}_{3}\right): \delta 170.7,170.2,130.0,124.6$, 95.6, 74.1, 69.5, 64.9, 64.1, 63.1, 30.7, 20.9, 20.7 ppm; IR (neat): 3436, 2971, 2357, 2225, 1739, 1444, 1375, 1227, $1037 \mathrm{~cm}^{-1}$; HRMS (Tof) $[\mathrm{M}+\mathrm{Na}]^{+}$: calcd. for $\mathrm{C}_{22} \mathrm{H}_{26} \mathrm{O}_{5} \mathrm{Na}$ 393.1678, found 393.1686. 
$[\alpha]_{\mathrm{D}}^{23}=+2.0^{\circ}\left(\mathrm{c}=1.0 \mathrm{CH}_{2} \mathrm{Cl}_{2}\right)$.

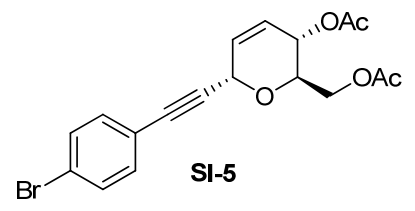

Alkynyl $\boldsymbol{C}$-glycoside SI-5: Zinc chloride (800 $\mathrm{mg} 5.9 \mathrm{mmol}$ ), was added to a flame-dried $80 \mathrm{~mL}$-microwave flask under an inert atmosphere (glove box). The flask was removed from the glove box and placed under argon. Dichloroethane $(10 \mathrm{~mL})$ was added, followed by $N, N$ - diethyl-1,1,1trimethylsilylamine $(0.800 \mathrm{~mL}, 4.0 \mathrm{mmol})$ and 4-bromophenylacetylene $(660 \mathrm{mg}, 3.7 \mathrm{mmol})$. The flask was sealed and heated using microwave irradiation $\left(150^{\circ} \mathrm{C}, 150-300 \mathrm{~W}\right.$, Powermax enabled) for $15 \mathrm{~min}$. The reaction was again placed under argon and transferred to a $-25^{\circ} \mathrm{C}$ cold bath. Tri-O-acetyl-D-glucal $(500 \mathrm{mg}, 1.8 \mathrm{mmol})$ and scandium triflate $(140 \mathrm{mg}, 0.2 \mathrm{mmol})$ were added. After $1.5 \mathrm{~h}$, the reaction was diluted with sat. $\mathrm{NaHCO}_{3}$ and extracted with $\mathrm{CH}_{2} \mathrm{Cl}_{2}$. The organic fraction was washed with sat. brine, dried, and concentrated. Chromatography over $\mathrm{SiO}_{2}(0-40 \%$ EtOAc / pet. ether) provided SI-5 (472 mg, 60\%) as a viscous oil. ${ }^{1} \mathrm{H}$ NMR (400 $\left.\mathrm{MHz}, \mathrm{CDCl}_{3}\right): \delta 7.46$ $(\mathrm{d}, 2 \mathrm{H}, J=8.4), 7.31$ (d, 2H, $J=8.8), 5.96(\mathrm{ddd}, 1 \mathrm{H}, J=1.6,3.6,10.0), 5.83(\mathrm{dt}, 1 \mathrm{H}, J=2.0,10.0), 5.34$ (dq, 1H, $J=2.0,8,8), 5.18(\mathrm{~m}, 1 \mathrm{H}), 4.26(\operatorname{appd}, 2 \mathrm{H}, J=4.4), 4.16(\mathrm{~m}, 1 \mathrm{H}), 2.10(\mathrm{~s}, 6 \mathrm{H}) \mathrm{ppm} ;{ }^{13} \mathrm{C}$ NMR $\left(100 \mathrm{MHz}, \mathrm{CDCl}_{3}\right): \delta 170.9,170.2,133.2,131.6,128.9,125.7,123.1,121.0,85.8,85.5,70.1,64.7$, 64.3, 62.9, 21.0, $20.8 \mathrm{ppm}$; IR (neat): 1746, 1487, 1371, 1229, 1044, $825 \mathrm{~cm}^{-1} ;[\alpha]_{\mathrm{D}}^{23}=-111^{\mathrm{o}}(\mathrm{c}=1.0$ $\left.\mathrm{CHCl}_{3}\right)$.

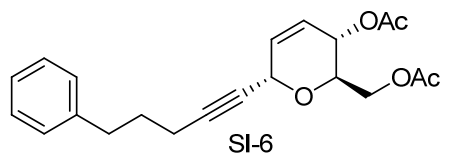

Alkynyl $\boldsymbol{C}$-glycoside SI-6: Zinc chloride (225 mg $1.7 \mathrm{mmol}$ ), was added to a flame-dried $80 \mathrm{~mL}$-microwave flask under an inert atmosphere (glove box). The flask was removed from the glove box and placed under argon. Dichloroethane $(10 \mathrm{~mL})$ was added, followed by $N, N$ - diethyl-1,1,1trimethylsilylamine $(0.330 \mathrm{~mL}, 1.6 \mathrm{mmol})$ and 5-phenyl-1-propyne $(0.220 \mathrm{~mL}, 1.4 \mathrm{mmol})$. The flask was sealed and heated using microwave irradiation $\left(150^{\circ} \mathrm{C}, 150-300 \mathrm{~W}\right.$, Powermax enabled) for 15 min. The reaction was again placed under argon and transferred to a $-25^{\circ} \mathrm{C}$ cold bath. Tri-O-acetyl-D-glucal $(250 \mathrm{mg}, 0.92 \mathrm{mmol})$ and scandium triflate $(68 \mathrm{mg}, 0.14 \mathrm{mmol})$ were added. After $1.5 \mathrm{~h}$, the reaction was diluted with sat. $\mathrm{NaHCO}_{3}$ and extracted with $\mathrm{CH}_{2} \mathrm{Cl}_{2}$. The organic fraction was washed with sat. brine, dried, and concentrated. Chromatography over $\mathrm{SiO}_{2}(0-40 \%$ EtOAc / pet. ether) provided SI-6 (237 mg, 72\%) as a viscous oil. ${ }^{1} \mathrm{H}$ NMR (400 $\left.\mathrm{MHz}, \mathrm{CDCl}_{3}\right): \delta 7.36$ $(\mathrm{m}, 2 \mathrm{H}), 7.26(\mathrm{~m}, 3 \mathrm{H}), 5.96(\mathrm{ddd}, 1 \mathrm{H}, J=2.0,3.2,10.2), 5.82(\mathrm{dt}, 1 \mathrm{H}, J=1.6,10.4), 3.56(\mathrm{dq}, 1 \mathrm{H}$, $J=2.0,8.8), 5.04(\mathrm{~m}, 1 \mathrm{H}), 4.23(\mathrm{~m}, 3 \mathrm{H}), 2.79(\mathrm{t}, 1 \mathrm{H}, J=6.8), 2.31(\mathrm{td}, 1 \mathrm{H}, J=2.0,7.2), 2.16(\mathrm{~s}, 3 \mathrm{H})$, $2.14(\mathrm{~s}, 3 \mathrm{H}), 1.91(\mathrm{~m}, 1 \mathrm{H}), \mathrm{ppm} ;{ }^{13} \mathrm{C} \mathrm{NMR}\left(100 \mathrm{MHz}, \mathrm{CDCl}_{3}\right): \delta 170.9,170.3,141.4,130.0,128.5$, 128.4, 126.0, 124.8, 87.3, 76.4, 69.7, 64.9, 64.2, 63.2, 34.7, 30.0, 21.0, 20.8, 18.2 ppm; IR (neat): 3429, $2936,2236,1740,1499,1363,1235,1052 \mathrm{~cm}^{-1} ;[\alpha]_{\mathrm{D}}^{23}=+13^{\circ}\left(\mathrm{c}=1.0 \mathrm{CHCl}_{3}\right)$.

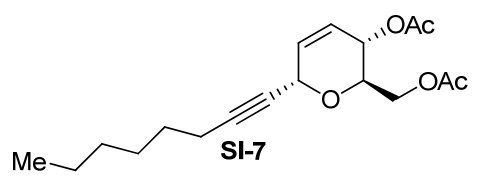

Alkynyl $\boldsymbol{C}$-glycoside SI-7: Zinc chloride (800 mg $5.9 \mathrm{mmol}$ ), was added to a flame-dried $80 \mathrm{~mL}$-microwave flask under an inert atmosphere (glove box). The flask was removed from the glove box and placed under argon. Dichloroethane $(10 \mathrm{~mL})$ was added, followed by 
$N, N$-diethyl-1,1,1-trimethylsilylamine $(0.800 \mathrm{~mL}, 4.0 \mathrm{mmol})$ and 1-octyne $(0.540 \mathrm{~mL}, 3.7 \mathrm{mmol})$. The flask was sealed and heated using microwave irradiation $\left(150^{\circ} \mathrm{C}, 150-300 \mathrm{~W}\right.$, Powermax enabled) for $15 \mathrm{~min}$. The reaction was again placed under argon and transferred to a $-25^{\circ} \mathrm{C}$ cold bath. Tri-O-acetyl-D-glucal $(500 \mathrm{mg}, 1.8 \mathrm{mmol})$ and scandium triflate $(90 \mathrm{mg}, 0.2 \mathrm{mmol}$ ) were added. After $1.5 \mathrm{~h}$, the reaction was diluted with sat. $\mathrm{NaHCO}_{3}$ and extracted with $\mathrm{CH}_{2} \mathrm{Cl}_{2}$. The organic fraction was washed with sat. brine, dried, and concentrated. Chromatography over $\mathrm{SiO}_{2}(0-40 \%$ EtOAc / pet. ether) provided SI-7 (458 mg, 77\%) as a viscous oil. $\left.{ }^{1} \mathrm{H} \mathrm{NMR} \mathrm{(400} \mathrm{MHz}, \mathrm{CDCl}_{3}\right): \delta$ 5.89 (ddd, $1 \mathrm{H}, J=1.4,3.4,10.2), 5.75$ (appd, $1 \mathrm{H}, J=10.4), 5.29$ (dt, $1 \mathrm{H}, J=2.0,8.8), 4.96$ (m, $1 \mathrm{H}), 4.22$ $(\mathrm{m}, 2 \mathrm{H}), 4.11(\mathrm{~m}, 1 \mathrm{H}), 2.23(\mathrm{td}, 2 \mathrm{H}, J=2.0,7.0), 2.10(\mathrm{~s}, 3 \mathrm{H}), 2.09(\mathrm{~s}, 3 \mathrm{H}), 1.51(\mathrm{~m}, 2 \mathrm{H}), 1.24-1.30(\mathrm{~m}$, $6 \mathrm{H}), 0.89$ (t, 3H, $J=6.4)$ ppm; ${ }^{13} \mathrm{C}$ NMR $\left(100 \mathrm{MHz}, \mathrm{CDCl}_{3}\right): \delta 170.1,170.0,129.9,124.5,87.6,76.7$, 75.6, 69.4, 64.7, 64.0, 62.9, 31.1, 28.2, 28.2, 22.3, 20.8, 20.6, 18.5, 13.8 ppm; IR (neat): 2924, 2858, $1744,1635,1371,1235,1049 \mathrm{~cm}^{-1} ;[\alpha]_{\mathrm{D}}^{23}=+5^{\circ}\left(\mathrm{c}=1.0 \mathrm{CHCl}_{3}\right)$.

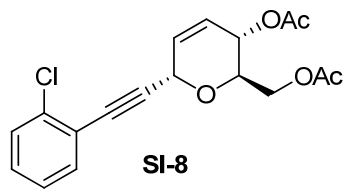

Alkynyl $\boldsymbol{C}$-glycoside SI-8: Zinc chloride (1.05 $\mathrm{g} 7.7 \mathrm{mmol})$, was added to a flame-dried $80 \mathrm{~mL}-$ microwave flask under an inert atmosphere (glove box). The flask was removed from the glove box and placed under argon. Dichloroethane $(10 \mathrm{~mL})$ was added, followed by $N, N-$ diethyl-1,1,1trimethylsilylamine $(1.20 \mathrm{~mL}, 6.1 \mathrm{mmol})$ and 1-chloro-2-ethynyl-benzene $(0.490 \mathrm{~mL}, 4.0 \mathrm{mmol})$. The flask was sealed and heated using microwave irradiation $\left(150^{\circ} \mathrm{C}, 150-300 \mathrm{~W}\right.$, Powermax enabled) for $15 \mathrm{~min}$. The reaction was again placed under argon and transferred to a $-25^{\circ} \mathrm{C}$ cold bath. Tri-O-acetyl-D-glucal $(550 \mathrm{mg}, 2.0 \mathrm{mmol})$ and scandium triflate $(100 \mathrm{mg}, 0.2 \mathrm{mmol})$ were added. After $1.5 \mathrm{~h}$ the reaction was diluted with sat. $\mathrm{NaHCO}_{3}$ and extracted with $\mathrm{CH}_{2} \mathrm{Cl}_{2}$. The organic fraction was washed with sat. brine, dried, and concentrated. Chromatography over $\mathrm{SiO}_{2}(0-40 \%$ EtOAc / pet. ether) provided SI-8 (244 mg, 35\%) as a viscous oil. ${ }^{1} \mathrm{H}$ NMR (400 $\left.\mathrm{MHz}, \mathrm{CDCl}_{3}\right): \delta$ 7.44 (ddd, 1H, $J=1.6,7.2,26), 7.26$ (m, 3H), 6.01 (ddd, 1H, $J=2.0,3.6,10.4$ ), 5.83 (dt, $1 \mathrm{H}, J=2.0$, 10.0), $5.34(\mathrm{~d}, 1 \mathrm{H}, J=5.2), 5.24(\mathrm{~m}, 1 \mathrm{H}), 4.27(\mathrm{~m}, 3 \mathrm{H}), 2.11(\mathrm{~s}, 3 \mathrm{H}), 2.11(\mathrm{~s}, 3 \mathrm{H}) \mathrm{ppm} ;{ }^{13} \mathrm{C}$ NMR $(100$ $\left.\mathrm{MHz}, \mathrm{CDCl}_{3}\right): \delta 170.7,170.2,136.0,133.1,129.6,129.2,128.7,126.3,125.7,122.0,89.9,83.3,70.1$, 64.6, 64.4, 63.0, 20.9, $20.7 \mathrm{ppm}$; IR (neat): 2354, 2336, 1743, 1473, 1364, 14229, $1051 \mathrm{~cm}^{-1} ;[\alpha]_{\mathrm{D}}^{23}=$ $-101^{\circ}\left(\mathrm{c}=1.0 \mathrm{CH}_{2} \mathrm{Cl}_{2}\right)$.

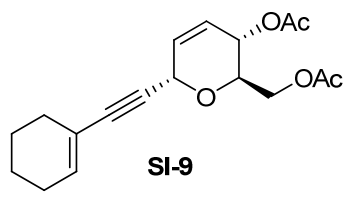

Alkynyl $\boldsymbol{C}$-glycoside SI-9: Zinc chloride (800 $\mathrm{mg} 5.9 \mathrm{mmol}$ ), was added to a flame-dried $80 \mathrm{~mL}-$ microwave flask under an inert atmosphere (glove box). The flask was removed from the glove box and placed under argon. Dichloroethane $(10 \mathrm{~mL})$ was added, followed by $N, N$ diethyl-1,1,1-trimethylsilylamine $(0.800 \mathrm{~mL}, 4.0 \mathrm{mmol})$ and 1-ethynylcyclohexane $(0.430 \mathrm{~mL}, 3.7$ $\mathrm{mmol})$. The flask was sealed and heated using microwave irradiation $\left(150^{\circ} \mathrm{C}, 150-300 \mathrm{~W}\right.$, Powermax enabled) for $15 \mathrm{~min}$. The reaction was again placed under argon and transferred to a $-25^{\circ} \mathrm{C}$ cold bath. Tri-O-acetyl-D-glucal $(500 \mathrm{mg}, 1.8 \mathrm{mmol})$ and scandium triflate $(100 \mathrm{mg}, 0.2 \mathrm{mmol})$ were added. After $1.5 \mathrm{~h}$, the reaction was diluted with sat. $\mathrm{NaHCO}_{3}$ and extracted with $\mathrm{CH}_{2} \mathrm{Cl}_{2}$. The organic fraction was washed with sat. brine, dried, and concentrated. Chromatography over $\mathrm{SiO}_{2}(0-40 \%$ 
EtOAc / pet. ether) provided SI-9 (372 mg, 60\%) as a viscous oil. ${ }^{1} \mathrm{H}$ NMR (400 MHz, $\left.\mathrm{CDCl}_{3}\right): \delta 6.12$ $(\mathrm{m}, 1 \mathrm{H}), 5.88(\mathrm{ddd}, 1 \mathrm{H}, J=2.0,3.4,10.1), 5.74(\mathrm{dt}, 1 \mathrm{H}, J=2.0,10.0), 5.28(\mathrm{~m}, 1 \mathrm{H}), 5.06(\mathrm{bs}, 1 \mathrm{H}), 4.21$ $(\mathrm{m}, 2 \mathrm{H}), 4.09(\mathrm{~m}, 1 \mathrm{H}), 2.06(\mathrm{~s}, 3 \mathrm{H}), 2.05(\mathrm{~m}, 10 \mathrm{H}), 1.61(\mathrm{~m}, 4 \mathrm{H}) \mathrm{ppm} ;{ }^{13} \mathrm{C} \mathrm{NMR}\left(100 \mathrm{MHz}, \mathrm{CDCl}_{3}\right): \delta$ $170.8,170.2$, 136.0, 130.4, 129.5, 125.0, 119.8, 88.5, 81.8, 74.4, 69.7, 65.7, 64.8, 64.4, 63.4, 63.0, 28.9, 29.8, 25.5, 22.1, 21.3, 21.0, 20.8 ppm; IR (neat): 3449, 2940, 2858, 1744, 1441, 1375, 1231, 1049, 917 $\mathrm{cm}^{-1} ;[\alpha]_{\mathrm{D}}^{23}=-86^{\circ}\left(\mathrm{c}=1.0 \mathrm{CHCl}_{3}\right)$.

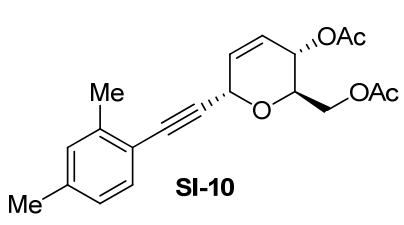

Alkynyl $\boldsymbol{C}$-glycoside SI-10: Zinc chloride (800 $\mathrm{mg}, 5.9 \mathrm{mmol})$, was added to a flame-dried $80 \mathrm{~mL}$-microwave flask under an inert atmosphere (glove box). The flask was removed from the glove box and placed under argon. Dichloroethane $(10 \mathrm{~mL})$ was added, followed by $N, N$ diethyl-1,1,1- trimethylsilylamine $(0.520 \mathrm{~mL}, 4.0 \mathrm{mmol})$ and 2-ethynyl-1,4-dimethylbenzene $(0.520 \mathrm{~mL}, 3.7 \mathrm{mmol})$. The flask was sealed and heated using microwave irradiation $\left(150^{\circ} \mathrm{C}, 150-300 \mathrm{~W}\right.$, Powermax enabled) for $15 \mathrm{~min}$. The reaction was again placed under argon and transferred to a $-25^{\circ} \mathrm{C}$ cold bath. Tri-O-acetyl-D-glucal $(500 \mathrm{mg}, 1.80 \mathrm{mmol})$ and scandium triflate $(90 \mathrm{mg}, 0.20 \mathrm{mmol}$ ) were added. After $5.0 \mathrm{~h}$ the reaction was diluted with sat. $\mathrm{NaHCO}_{3}$ and extracted with $\mathrm{CH}_{2} \mathrm{Cl}_{2}$. The organic fraction was washed with sat. brine, dried, and concentrated. Chromatography over $\mathrm{SiO}_{2}(0-40 \%$ EtOAc / pet. ether) provided SI-10 (554 mg, 88\%) as a viscous oil. ${ }^{1} \mathrm{H}$ NMR $\left(400 \mathrm{MHz}, \mathrm{CDCl}_{3}\right): \delta 7.23(\mathrm{~s}, 1 \mathrm{H}), 7.05(\mathrm{~m}, 2 \mathrm{H}), 5.99(\mathrm{dq}, 1 \mathrm{H}, J=1.6,3.6$, 10.4), 5.81 (dt, 1H, $J=2.0,10.0), 5.33(\mathrm{dd}, 1 \mathrm{H}, J=2.0,8.8), 5.23(\mathrm{~m}, 1 \mathrm{H}), 4.25(\mathrm{~m}, 3 \mathrm{H}), 2.38(\mathrm{~s}, 3 \mathrm{H})$, $2.28(\mathrm{~s}, 3 \mathrm{H}), 2.10(\mathrm{~s}, 3 \mathrm{H}), 2.09(\mathrm{~s}, 3 \mathrm{H}) \mathrm{ppm} ;{ }^{13} \mathrm{C} \mathrm{NMR}\left(100 \mathrm{MHz}, \mathrm{CDCl}_{3}\right): \delta 170.9,170.3,137.2$, 135.0, 132.4, 129.6, 129.4, 129.3, 125.4, 121.7, 88.1, 85.9, 70.0, 64.9, 64.6, 63.2, 21.0, 20.8, 20.7, 20.1 ppm; IR (neat): $3390,2916,1736,1449,1375,1227,1041 \mathrm{~cm}^{-1} ;[\alpha]_{\mathrm{D}}^{23}=-15^{\circ}\left(\mathrm{c}=1.0 \mathrm{CHCl}_{3}\right)$.

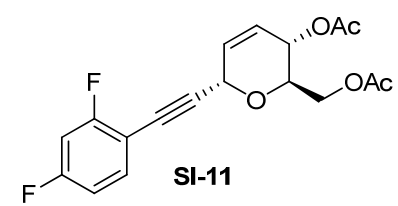

Alkynyl $\boldsymbol{C}$-glycoside SI-11: Zinc chloride (800 mg, $5.9 \mathrm{mmol})$, was added to a flame-dried $80 \mathrm{~mL}$-microwave flask under an inert atmosphere (glove box). The flask was removed from the glove box and placed under argon. Dichloroethane $(10 \mathrm{~mL})$ was added, followed by $N, N$ - diethyl-1,1,1trimethylsilylamine $\quad(0.800 \quad \mathrm{~mL}, \quad 4.0 \quad \mathrm{mmol}) \quad$ and 1-ethynyl-2,4-difluorobenzene $(510 \mathrm{mg}, 3.7 \mathrm{mmol})$. The flask was sealed and heated using microwave irradiation $\left(150^{\circ} \mathrm{C}, 150-300 \mathrm{~W}\right.$, Powermax enabled) for $15 \mathrm{~min}$. The reaction was again placed under argon and transferred to a $-25^{\circ} \mathrm{C}$ cold bath. Tri-O-acetyl-D-glucal $(500 \mathrm{mg}, 1.80 \mathrm{mmol})$ and scandium triflate $(90 \mathrm{mg}, 0.20 \mathrm{mmol}$ ) were added. After $5.0 \mathrm{~h}$ the reaction was diluted with sat. $\mathrm{NaHCO}_{3}$ and extracted with $\mathrm{CH}_{2} \mathrm{Cl}_{2}$. The organic fraction was washed with sat. brine, dried, and concentrated. Chromatography over $\mathrm{SiO}_{2}(0-40 \%$ EtOAc / pet. ether) provided SI-11 (316 mg, 49\%) as a viscous oil. ${ }^{1} \mathrm{H}$ NMR (400 MHz, $\left.\mathrm{CDCl}_{3}\right): \delta 7.41(\mathrm{~m}, 1 \mathrm{H}), 6.85(\mathrm{~m}, 2 \mathrm{H}),, 5.97(\mathrm{dq}, 1 \mathrm{H}, J=1.6,3.6$, 10.4), 5.84 (dt, 1H, $J=2.0,10.4), 5.34$ (dq, $1 \mathrm{H}, J=2.0,4.0,8.8), 5.21(\mathrm{~m}, 1 \mathrm{H}), 4.25(\mathrm{~m}, 2 \mathrm{H}), 4.18(\mathrm{~m}$, $1 \mathrm{H}), 2.11(\mathrm{~s}, 3 \mathrm{H}), 2.10(\mathrm{~s}, 3 \mathrm{H}) \mathrm{ppm} ;{ }^{13} \mathrm{C} \mathrm{NMR}\left(100 \mathrm{MHz}, \mathrm{CDCl}_{3}\right): \delta 170.9,170.3,164.4(\mathrm{dd}, 1 \mathrm{C}$, $J=46.8,134.8), 161.9(\mathrm{dd}, 1 \mathrm{C}, J=47.2,122.8), 134.5$ (t, 1C, $J=12), 128.7,125.9$, $111.6(\mathrm{dd}, 1 \mathrm{C}$, $J=14.8,84.8$ ), 107.1 (d, 1C, $J=46.8$ ), 99.6 (t, 1C, $J=99.6), 89.7,79.1,70.2,64.7,64.4,63.0,21.0,20.8$ ppm; IR (neat): 1743, 1623, 1510, 1425, 1375, 1239, $1052 \mathrm{~cm}^{-1}$; HRMS (Tof) [M+Na] $]^{+}$calcd. for 
$\mathrm{C}_{18} \mathrm{H}_{16} \mathrm{O}_{5} \mathrm{NaF}_{2}$ 373.0864, found 373.0799. $[\alpha]_{\mathrm{D}}^{23}=-88^{\circ}\left(\mathrm{c}=1.0 \mathrm{CHCl}_{3}\right)$.

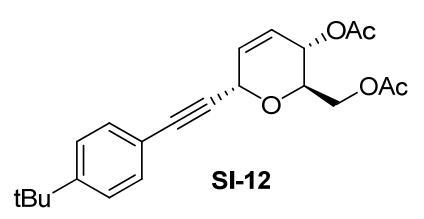

Alkynyl $\boldsymbol{C}$-glycoside SI-12: Zinc chloride (800 mg, $5.9 \mathrm{mmol})$, was added to a flame-dried $80 \mathrm{~mL}-$ microwave flask under an inert atmosphere (glove box). The flask was removed from the glove box and placed under argon. Dichloroethane $(10 \mathrm{~mL})$ was added, followed by $N, N$ - diethyl-1,1,1trimethylsilylamine $\quad(0.800 \quad \mathrm{~mL}, \quad 4.0 \quad \mathrm{mmol}) \quad$ and 1-tert-butyl-4-ethynylbenzene $(0.660 \mathrm{~mL}, 3.7 \mathrm{mmol})$. The flask was sealed and heated using microwave irradiation $\left(150^{\circ} \mathrm{C}, 150-300 \mathrm{~W}\right.$, Powermax enabled) for $15 \mathrm{~min}$. The reaction was again placed under argon and transferred to a $-25^{\circ} \mathrm{C}$ cold bath. Tri-O-acetyl-D-glucal $(500 \mathrm{mg}, 1.80 \mathrm{mmol})$ and scandium triflate $(90 \mathrm{mg}, 0.20 \mathrm{mmol}$ ) were added. After $5.0 \mathrm{~h}$ the reaction was diluted with sat. $\mathrm{NaHCO}_{3}$ and extracted with $\mathrm{CH}_{2} \mathrm{Cl}_{2}$. The organic fraction was washed with sat. brine, dried, and concentrated. Chromatography over $\mathrm{SiO}_{2}(0-40 \%$ EtOAc / pet. ether) provided SI-12 (289 mg, 42\%) as a viscous oil. ${ }^{1} \mathrm{H}$ NMR (400 MHz, $\left.\mathrm{CDCl}_{3}\right): \delta 7.38(\mathrm{~d}, 2 \mathrm{H}, J=8.4), 7.33(\mathrm{~d}, 2 \mathrm{H}, J=9.2), 4.97(\mathrm{dq}, 1 \mathrm{H}$, $J=2.0,3.6,10.0), 5.81(\mathrm{dt}, 1 \mathrm{H}, J=2.0,10.0), 5.33(\mathrm{dq}, 1 \mathrm{H}, J=2.0,4.0,9.2), 5.18(\mathrm{~m}, 1 \mathrm{H}), 4.26(\mathrm{~d}, 2 \mathrm{H}$, $J=3.6), 4.20(\mathrm{~m}, 1 \mathrm{H}), 2.10(\mathrm{~s}, 3 \mathrm{H}), 2.09(\mathrm{~s}, 3 \mathrm{H}), 1.30(\mathrm{~s}, 9 \mathrm{H}) \mathrm{ppm} ;{ }^{13} \mathrm{C} \mathrm{NMR}\left(100 \mathrm{MHz}, \mathrm{CDCl}_{3}\right): \delta$ $170.9,170.3,152.1,131.5,129.3,125.3,119.1,86.8,84.0,69.9,64.8,64.5,63.0,34.8,31.1,21.0,20.8$ ppm; IR (neat): $2967,1740,1375,1243,1052,834 \mathrm{~cm}^{-1} ;[\alpha]_{\mathrm{D}}^{23}=-33^{\circ}\left(\mathrm{c}=1.0 \mathrm{CHCl}_{3}\right)$.

\section{Phenol addition to allyl-C-glycoside 2a}

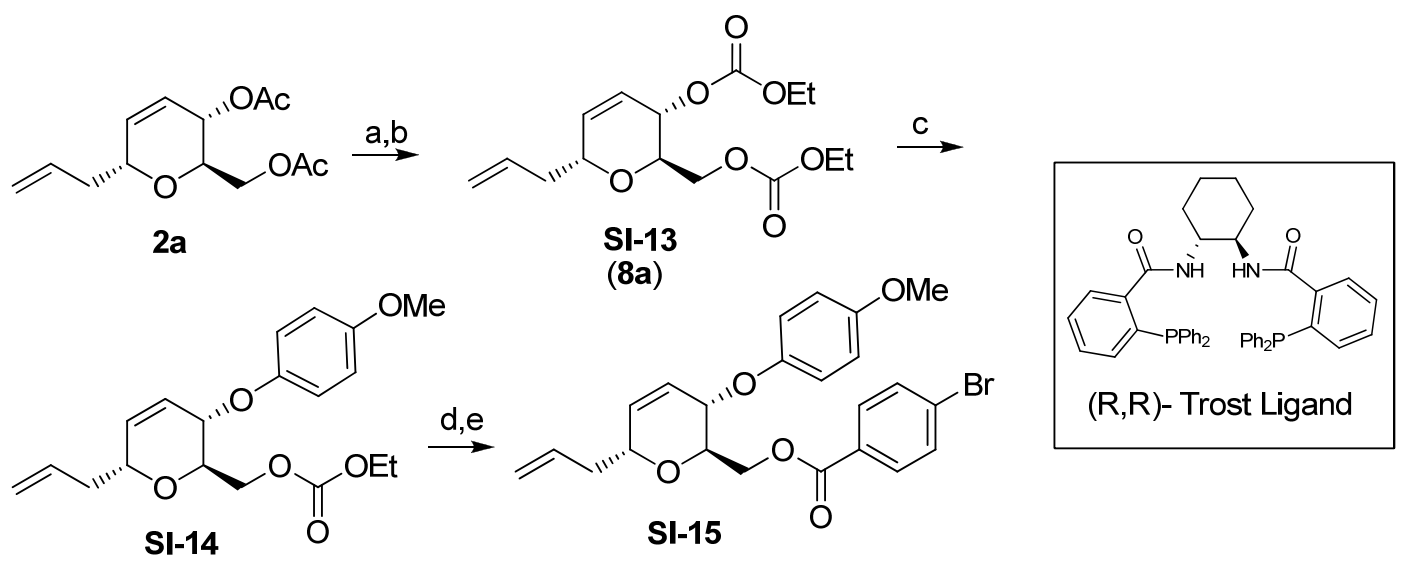

Scheme SI-1. C-glycoside aryl ether formation. a) $\mathrm{MP}_{-} \mathrm{CO}_{3}, \mathrm{MeOH},(99 \%)$; b) ethyl chloroformate, pyridine, DMAP, $\mathrm{CH}_{2} \mathrm{Cl}_{2}$, (81\%); c) $\mathrm{Pd}_{2}(\mathrm{dba})_{3}-\mathrm{CHCl}_{3}$, Trost Ligand, $p$-methoxyphenol, $\mu$ wave, $100^{\circ} \mathrm{C}(78 \%)$; d) $\mathrm{MP}-\mathrm{CO}_{3}, \mathrm{MeOH}(99 \%)$, e) $p-\mathrm{BrBnCl}, \mathrm{NEt}_{3}, \mathrm{DMAP}, \mathrm{CH}_{2} \mathrm{Cl}_{2}(\mathrm{X} \%)$

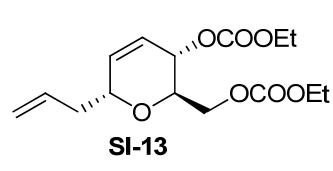

Dicarbonate SI-13: Diacetate $\mathbf{2 a}^{2}(708 \mathrm{mg}, 2.8 \mathrm{mmol})$ was dissolved in $\mathrm{MeOH}(4.0 \mathrm{~mL})$ and shaken with $\mathrm{MP}$-carbonate resin $(230 \mathrm{mg}, 0.70 \mathrm{mmol})$ for Sl-13 $12 \mathrm{~h}$. The reaction was filtered, rinsing with $\mathrm{MeOH}$, then concentrated in

\footnotetext{
2 S. Su, D.E. Acquilano, J. Arumugasamy, A.B. Beeler, E.L. Eastwood, J.R. Giguere, P. Lan, X. Lei, G.K. Min, A.R. Yeager, Y. Zhou, J.S. Panek, J.K. Snyder, S.E. Schaus, J.A. Porco Jr., Org Lett. 2005, 7, 2751-2754.
} 
vacuo. The crude residue was redissolved in methylene chloride $(3.0 \mathrm{~mL})$ and pyridine $(0.68 \mathrm{~mL}$, $8.5 \mathrm{mmol})$ was added. The reaction was cooled to $0^{\circ} \mathrm{C}$ and ethyl chloroformate $(0.81 \mathrm{~mL}, 8.5 \mathrm{mmol})$ was added. After stirring for $12 \mathrm{~h}$, the reaction was diluted with methylene chloride and washed with sat. aqueous sodium bicarbonate and brine. The organic layer was dried (sodium sulfate), concentrated, and chromatographed over $\mathrm{SiO}_{2}(0-40 \%$ ethyl acetate / petroleum ether) to provide SI-13 $(715 \mathrm{mg}, 81 \%)$ as a viscous oil. ${ }^{1} \mathrm{H}$ NMR $\left(400 \mathrm{MHz}, \mathrm{CDCl}_{3}\right): \delta 5.95(1 \mathrm{H}, \mathrm{dt}, J=12.0,2.0)$, $5.87(2 \mathrm{H}, \mathrm{m}), 5.14(1 \mathrm{H}$, appd, $J=1.6), 5.10(1 \mathrm{H}, \mathrm{m}), 5.02(1 \mathrm{H}, \mathrm{m}), 4.23(7 \mathrm{H}, \mathrm{m}), 4.02(1 \mathrm{H}, \mathrm{q}, J=4.4)$, $2.45(1 \mathrm{H}, \mathrm{m}), 2.33(1 \mathrm{H}, \mathrm{m}), 1.31(6 \mathrm{H}, \mathrm{m}) \mathrm{ppm} ;{ }^{13} \mathrm{C} \mathrm{NMR}\left(100 \mathrm{MHz}, \mathrm{CDCl}_{3}\right): \delta 155.0,154.5,138.8$, 133.2, 126.2, 117.7, 71.5, 69.2, 68.1, 66.0, 61.3, 64.2, 37.7, 14.2, 14.2 ppm; IR (neat): 3740, 3414, $2978,2912,1961,1748,1375,1258,1314 \mathrm{~cm}^{-1} ;[\alpha]_{\mathrm{D}}^{23}=+50^{\circ}\left(\mathrm{c}=1.0 \mathrm{CH}_{2} \mathrm{Cl}_{2}\right)$.

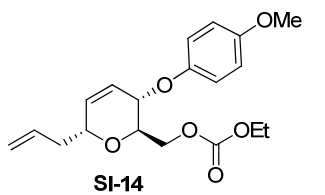

Aryl ether SI-14: Dicarbonate SI-13 (508 mg, $1.62 \mathrm{mmol})$, Tris (dibenzylideneacetone) dipalladium (0) chloroform adduct ( $42 \mathrm{mg}, 0.04 \mathrm{mmol}$ ), and $(1 S, 2 S)$ Trost ligand $(56 \mathrm{mg}, 0.08 \mathrm{mmol})$ were added to a flame-dried 10 $\mathrm{mL}$-microwave vessel and were dissolved in degassed dichloromethane $(2 \mathrm{~mL})$.

After $30 \mathrm{~min} p$-methoxyphenol $(221 \mathrm{mg}, 1.78 \mathrm{mmol})$ was added and the mixture was heated using microwave irradiation $\left(300 \mathrm{~W}\right.$, Powermax enabled) at $100^{\circ} \mathrm{C}$ for $15 \mathrm{~min}$. The reaction was concentrated and purified over $\mathrm{SiO}_{2}(0-40 \%$ ethyl acetate/ petroleum ether) to provide SI-14 (441 mg, $1.27 \mathrm{mmol}, 78 \%)$ as a viscous oil. ${ }^{1} \mathrm{H}$ NMR $\left(400 \mathrm{MHz} ; \mathrm{CDCl}_{3}\right): \delta 6.85(4 \mathrm{H}, \mathrm{m}), 5.96(1 \mathrm{H}, \mathrm{m}), 5.90$ $(2 \mathrm{H}, \mathrm{m}), \quad 5.13(2 \mathrm{H}, \mathrm{m}), 4.60(1 \mathrm{H}, \mathrm{dd}, J=1.6,8), 4.40(1 \mathrm{H}, \mathrm{dd}, J=2.8,11.4), 4.32(2 \mathrm{H}, \mathrm{m}), 4.17$ $(2 \mathrm{H}, \mathrm{q}), 4.15(1 \mathrm{H}, \mathrm{m}), 3.76(3 \mathrm{H}, \mathrm{s}), 2.52(1 \mathrm{H}, \mathrm{m}), 2.36(1 \mathrm{H}, \mathrm{m}), 1.28(3 \mathrm{H}, \mathrm{t}) \mathrm{ppm} ;{ }^{13} \mathrm{C}$ NMR $(100$ $\left.\mathrm{MHz}, \mathrm{CDCl}_{3}\right): \delta 155.1,154.3,121.0,134.1,131.6,124.6,117.5,117.0,114.7,72.5,69.4,69.4,66.7$, 64.0, 55.7, 37.7, 14.2 ppm.; IR (neat): 2907, 1747, 1506, 1226, 1038, $828 \mathrm{~cm}^{-1} ;[\alpha]_{\mathrm{D}}^{23}=+81.9^{\circ}$ (c=1.0 $\mathrm{CH}_{2} \mathrm{Cl}_{2}$ ).

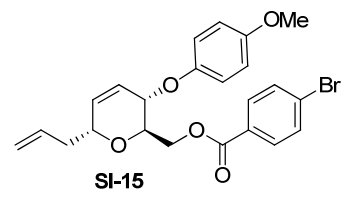

p-Bromobenzoate ester SI-15: Ethyl carbonate SI-14 was dissolved in $\mathrm{MeOH}$ and MP-carbonate was added. The reaction was stirred for $5 \mathrm{~h}$ and filtered to provide the unprotected alchol as a white solid. ${ }^{1} \mathrm{H}$ NMR (400 MHz; $\left.\mathrm{CDCl}_{3}\right)$ : $\delta 6.85(4 \mathrm{H}, \mathrm{m}), 6.94(1 \mathrm{H}, \mathrm{m}), 5.86(1 \mathrm{H}, \mathrm{m}), \quad 5.18(1 \mathrm{H}, \mathrm{q}), 5.12(1 \mathrm{H}, \mathrm{dd}, J=$ 3.2, 9.2), $4.63(1 \mathrm{H}, \mathrm{dd}, J=1.6,7.6), 4.31\left(1 \mathrm{H}^{+}, \mathrm{m}\right), 3.86(1 \mathrm{H}, \mathrm{dt}, J=2.4,14), 3.76(2 \mathrm{H}, \mathrm{m}), 3.77(3 \mathrm{H}$, s), $2.53(1 \mathrm{H}, \mathrm{m}), 2.34(1 \mathrm{H}, \mathrm{m}), 2.10(1 \mathrm{H}, \mathrm{bs}) ;{ }^{13} \mathrm{C} \mathrm{NMR}\left(100 \mathrm{MHz}, \mathrm{CDCl}_{3}\right): \delta 154.3,151.3,134.4$, 131.2, 125.3, 117.5, 117.1, 114.8, 72.6, 71.2, 69.5, 62.4, 55.7, 37.7 ppm; IR (neat): 3459, 2912, 1506, $1225,1040,828 \mathrm{~cm}^{-1}$; HRMS (Tof) [M+Na $]^{+}$: calcd. for $\mathrm{C}_{16} \mathrm{H}_{20} \mathrm{O}_{4} \mathrm{Na}$ 277.1416, found 277.1417. $[\alpha]_{\mathrm{D}}^{23}=+113.3^{\circ}\left(\mathrm{c}=1.0 \mathrm{CH}_{2} \mathrm{Cl}_{2}\right)$. The diol $(150 \mathrm{mg}, 0.54 \mathrm{mmol})$ was dissolved in dichloromethane. Triethylamine $(0.11 \mathrm{~mL}, 0.81 \mathrm{mmol})$ and dimethylaminopyridine $(7 \mathrm{mg}, 0.05 \mathrm{mmol})$ were added, the reaction was cooled to $0^{\circ} \mathrm{C}$ and 4-bromo-benzoyl chloride $(179 \mathrm{mg}, 0.81 \mathrm{mmol})$ was added. After $10 \mathrm{~min}$ the reaction was warmed to room temp and stirred for $4 \mathrm{~h}$. The solution was diluted with dichloromethane, washed with sat. aqueous sodium bicarbonate (1X) and brine (1X), filtered and purified over $\mathrm{SiO}_{2}$ to yield SI-15 (172 $\left.\mathrm{mg}, 0.37 \mathrm{mmol}\right)$ as a white solid. ${ }^{1} \mathrm{H}$ NMR $(400 \mathrm{MHz}$; $\left.\mathrm{CDCl}_{3}\right): \delta 7.90(2 \mathrm{H}, \mathrm{m}), 7.57(2 \mathrm{H}, \mathrm{m}), 6.84(4 \mathrm{H}, \mathrm{m}), 5.99(1 \mathrm{H}, \mathrm{m}), 5.89(2 \mathrm{H}, \mathrm{m}), 4.61(2 \mathrm{H}, \mathrm{m}), 4.45$ 
$(1 \mathrm{H}, \mathrm{q}), \quad 4.35\left(1 \mathrm{H}^{+}, \mathrm{m}\right), 4.13(1 \mathrm{H}, \mathrm{td}, J=2.8,7.2,14.4), 3.76(3 \mathrm{H}, \mathrm{s}), 2.54(1 \mathrm{H}, \mathrm{m}), 2.38(1 \mathrm{H}, \mathrm{m})$; ${ }^{13} \mathrm{C}$ NMR $\left(100 \mathrm{MHz}, \mathrm{CDCl}_{3}\right): \delta 165.0,154.4,151.1,134.3,131.8,131.2,128.9,128.1,124.7,117.5$, $117.1,114.8,72.5,70.0,70.0,64.6,55.7,55.7,37.6$ ppm; IR (neat): 2903, 1723, 1505, 1226, 1101, $827,753 \mathrm{~cm}^{-1} ; \quad[\alpha]_{\mathrm{D}}^{23}=64.8^{\circ}\left(\mathrm{c}=1.0 \mathrm{CH}_{2} \mathrm{Cl}_{2}\right)$.

\section{General procedure for synthesis of aryl $C$-glycosides $3 b$ (Table S2)}
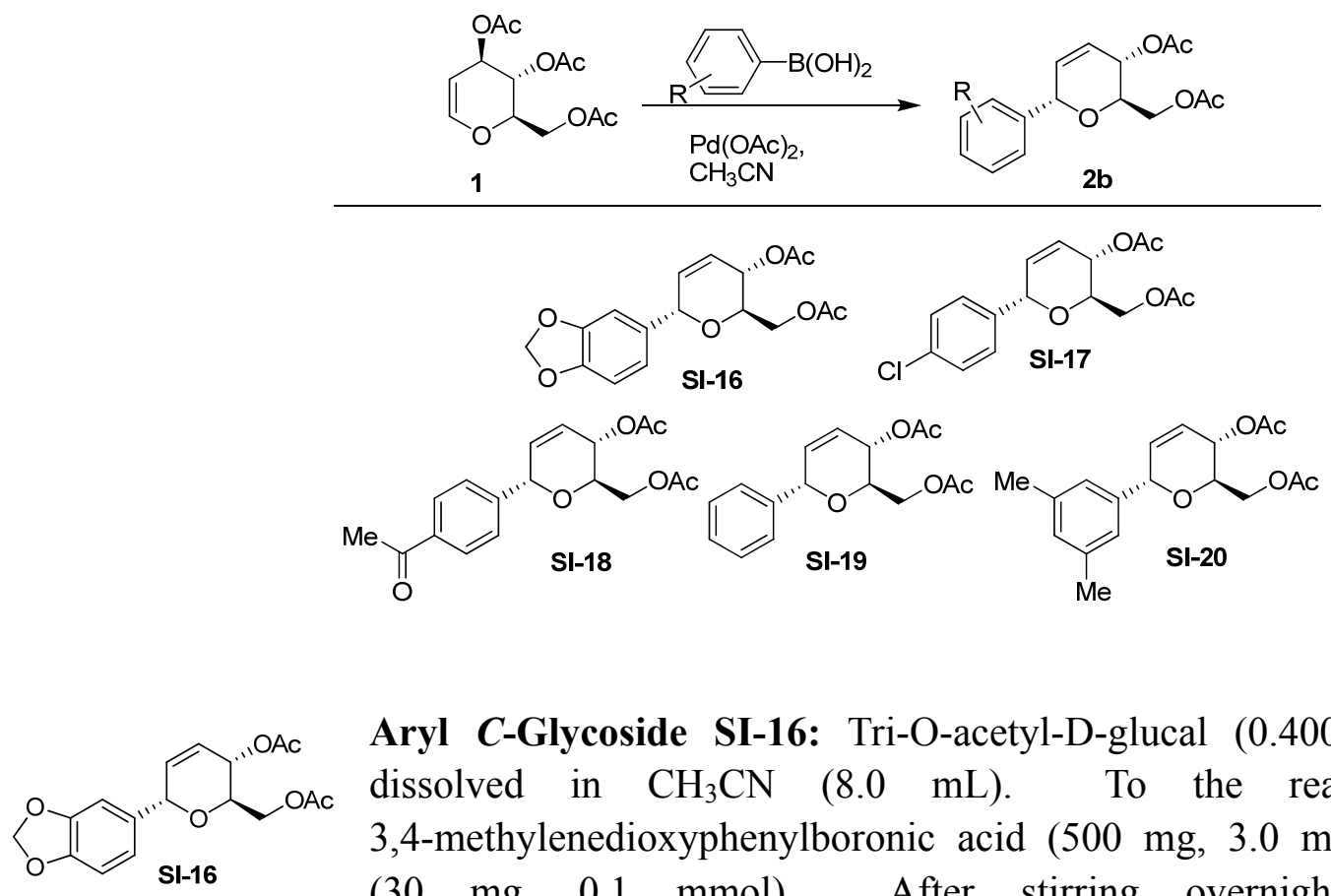

Aryl $\boldsymbol{C}$-Glycoside SI-16: Tri-O-acetyl-D-glucal $(0.400 \mathrm{~g}, 1.0 \mathrm{mmol})$ was dissolved in $\mathrm{CH}_{3} \mathrm{CN}(8.0 \mathrm{~mL})$. To the reaction was added 3,4-methylenedioxyphenylboronic acid $(500 \mathrm{mg}, 3.0 \mathrm{mmol})$ and $\mathrm{Pd}(\mathrm{OAc})_{2}$ (30 $\mathrm{mg}, \quad 0.1 \mathrm{mmol}$ ). After stirring overnight, additional 3,4methylenedioxyphenylboronic acid $(100 \mathrm{mg}, 0.6 \mathrm{mmol})$ and $\mathrm{Pd}(\mathrm{OAc})_{2}(20 \mathrm{mg}, 0.09 \mathrm{mmol})$ were added. After $18 \mathrm{~h}$, the reaction was concentrated onto $\mathrm{SiO}_{2}$ and chromatographed over $\mathrm{SiO}_{2}$, eluting with $0-60 \%$ ethyl acetate / pet. ether to provide SI-16 (338 mg, 70\%) as a viscous oil. ${ }^{1} \mathrm{H}$ NMR (400 $\left.\mathrm{MHz}, \mathrm{CDCl}_{3}\right): \delta 6.92(\mathrm{bs}, 1 \mathrm{H}), 6.86(\mathrm{~d}, 1 \mathrm{H}, J=7.6), 6.79(\mathrm{~d}, 1 \mathrm{H}, J=8.0), 6.11(\mathrm{~m}, 1 \mathrm{H}), 5.97(\mathrm{~m}, 3 \mathrm{H})$, $5.30(\mathrm{~m} \mathrm{1H}), 5.22(\mathrm{~m}, 1 \mathrm{H}), 4.26(\mathrm{dd}, 1 \mathrm{H}, J=6.0,12.0), 4.07$ (dd, $1 \mathrm{H}, J=3.2,12.0), 3.83(\mathrm{~m}, 1 \mathrm{H}), 2.09$ (s, 3H), 2.07 (s. 3H) ppm; $\left.{ }^{13} \mathrm{C} \mathrm{NMR} \mathrm{(100} \mathrm{MHz,} \mathrm{CDCl}_{3}\right): \delta 170.8,170.5,147.8,147.5,132.6,131.5$, 125.0, 121.7, 108.5, 108.0, 73.5, 69.0, 64.9, 62.8, 21.1, 20.8 ppm; IR (neat): 2889, 1740, 1499, 1491, $1445,1371,1231,1033,936,792 \mathrm{~cm}^{-1} ;[\alpha]_{\mathrm{D}}^{23}=+10^{\circ}\left(\mathrm{c}=1.0 \mathrm{CH}_{2} \mathrm{Cl}_{2}\right)$.

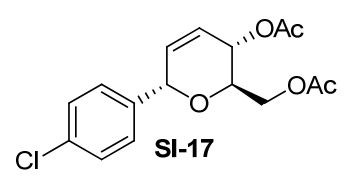

Aryl $\boldsymbol{C}$-Glycoside SI-17: Tri-O-acetyl-D-glucal (400 mg, $1.0 \mathrm{mmol}$ ) was dissolved in $\mathrm{CH}_{3} \mathrm{CN}(8.0 \mathrm{~mL})$. To the reaction was added 4-chlorophenylboronic acid (400 mg, $3.0 \mathrm{mmol})$ and $\mathrm{Pd}(\mathrm{OAc})_{2}(100 \mathrm{mg}, 0.4$ $\mathrm{mmol})$. After stirring overnight, additional $\mathrm{Pd}(\mathrm{OAc})_{2}(50 \mathrm{mg}, 0.2 \mathrm{mmol})$ was added. After $24 \mathrm{~h}$, the reaction was concentrated onto $\mathrm{SiO}_{2}$ and chromatographed over $\mathrm{SiO}_{2}$, eluting with $0-50 \%$ ethyl acetate / pet. ether to provide SI-17 $(0.241 \mathrm{~g}, 50 \%)$ as a viscous oil. ${ }^{3}$

3 (a) J. Ramnauth, O. Poulin, S. Rakhit, S.P. Maddaford, Org. Lett. 2001, 3, 2013-2015; (b) N. de la Figuera, P. Forns, J.C. Fernandez, S. Fiol, D. 


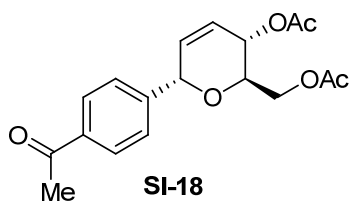

Aryl $\boldsymbol{C}$-Glycoside SI-18: Tri-O-acetyl-D-glucal $(400 \mathrm{mg}, 1.0 \mathrm{mmol})$ was dissolved in $\mathrm{CH}_{3} \mathrm{CN}(8.0 \mathrm{~mL})$. To the reaction was added 4-acetylphenylboronic acid $(700 \mathrm{mg}, 4.0 \mathrm{mmol})$ and $\mathrm{Pd}(\mathrm{OAc})_{2}(100 \mathrm{mg}, 0.4$ mmol). After stirring overnight, additional 4-acetylphenylboronic acid (200 $\mathrm{mg}, 1.2 \mathrm{mmol})$ and $\mathrm{Pd}(\mathrm{OAc})_{2}(100 \mathrm{mg}, 0.4 \mathrm{mmol})$ were added. After $24 \mathrm{~h}$, the reaction was concentrated onto $\mathrm{SiO}_{2}$ and chromatographed $\left(\mathrm{SiO}_{2}\right)$, eluting with $0-60 \%$ ethyl acetate / pet. ether to provide SI-18 (363 mg, 70\%) as a viscous oil. ${ }^{1} \mathrm{H}$ NMR $\left(400 \mathrm{MHz}, \mathrm{CDCl}_{3}\right): \delta 7.95(\mathrm{~d}, 2 \mathrm{H}, J=12.4)$, 7.49 (d, $2 \mathrm{H}, J=8.0), 6.18$ (ddd, $1 \mathrm{H}, J=1.6,2.8,10.4), 6.01(\mathrm{dt}, 1 \mathrm{H}, J=2.4,5.2), 5.34(\mathrm{~m}, 1 \mathrm{H}), 5.27$ (m, $1 \mathrm{H}), 4.26(\mathrm{dd}, 1 \mathrm{H}, J=6.4,12), 4.09(\mathrm{~m}, 1 \mathrm{H}), 3.81(\mathrm{~m}, 1 \mathrm{H}) 2.59(\mathrm{~s}, 3 \mathrm{H}), 2.07(\mathrm{~s}, 3 \mathrm{H}), 2.06(\mathrm{~s}, 3 \mathrm{H}) \mathrm{ppm}$; ${ }^{13} \mathrm{C}$ NMR $\left(100 \mathrm{MHz}, \mathrm{CDCl}_{3}\right): \delta 197.6,170.7,170.3,144.1,136.7,130.7,128.5,127.7,125.4,73.0$, 69.7, 64.7, 62.7, 26.6, 20.8 ppm; IR (neat): 2357, 1748, 1682, 1608, 1409, 1367, 1227, $1045 \mathrm{~cm}^{-1}$; $[\alpha]_{\mathrm{D}}^{23}=-7^{\circ}\left(\mathrm{c}=1.0 \mathrm{CHCl}_{3}\right)$.

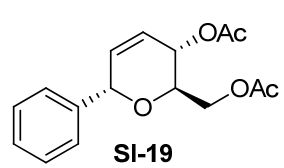

Aryl $\boldsymbol{C}$-Glycoside SI-19: Tri-O-acetyl-D-glucal $(2.00 \mathrm{~g}, 7.3 \mathrm{mmol})$ was dissolved in $\mathrm{CH}_{3} \mathrm{CN}(40 \mathrm{~mL})$. Into the reaction was added phenylboronic acid $(4.40 \mathrm{~g}, 36.0$ $\mathrm{mmol})$ and $\mathrm{Pd}(\mathrm{OAc})_{2}(450 \mathrm{mg}, 2.0 \mathrm{mmol})$. After $24 \mathrm{~h}$, the reaction was concentrated onto $\mathrm{SiO}_{2}$ and chromatographed over $\mathrm{SiO}_{2}$, eluting with $0-60 \%$ ethyl acetate / pet. ether to provide SI-16 (1.76 g, 82\%) as a viscous oil. ${ }^{3}$

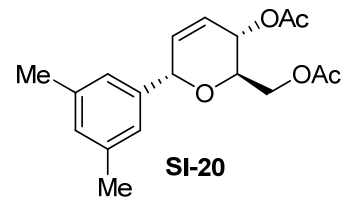

Aryl $\boldsymbol{C}$-Glycoside SI-20: Tri-O-acetyl-D-glucal $(750 \mathrm{mg}, 2.8 \mathrm{mmol}$ ) was dissolved in $\mathrm{CH}_{3} \mathrm{CN}(30.0 \mathrm{~mL})$. To the reaction was added 3,5-dimethylbenzene boronic acid (1200 $\mathrm{mg}, 8.3 \mathrm{mmol})$ and $\mathrm{Pd}(\mathrm{OAc})_{2}$ (200 mg, $0.8 \mathrm{mmol}$ ). After $12 \mathrm{~h}$, the reaction was concentrated onto $\mathrm{SiO}_{2}$ and chromatographed $\left(\mathrm{SiO}_{2}\right)$, eluting with $0-60 \%$ ethyl acetate / pet. ether to provide SI-20 $(756 \mathrm{mg}, 86 \%)$ as a viscous oil. ${ }^{1} \mathrm{H}$ NMR $\left(400 \mathrm{MHz}, \mathrm{CDCl}_{3}\right): \delta 7.01(\mathrm{~s}, 2 \mathrm{H})$, 6.95 (s, $1 \mathrm{H}), 6.17$ (dq, $1 \mathrm{H}, J=1.6,3.2,10.8), 5.96(\mathrm{dt}(1 \mathrm{H}, J=2.0,10.4), 5.28(\mathrm{~m}, 2 \mathrm{H}), 4.27(\mathrm{dd}, 1 \mathrm{H}$, $J=6.4,12.4), 4.10$ (dd, $1 \mathrm{H}, J=3.2,12.0), 3.86(\mathrm{~m}, 1 \mathrm{H}), 2.32(\mathrm{~s}, 6 \mathrm{H}), 2.08(\mathrm{~s}, 3 \mathrm{H}), 2.08$ (s, 3H) ppm; ${ }^{13} \mathrm{C}$ NMR $\left(100 \mathrm{MHz}, \mathrm{CDCl}_{3}\right): \delta 170.7,170.3,138.6,137.9,131.7,129.7,125.5,124.6,73.5,69.3$, 65.0, 62.8, 21.2, 20.9, 20.7 ppm; IR (neat): 2913, 1744, 1604, 1441, 1367, 1235, $1045 \mathrm{~cm}^{-1}$; HRMS (Tof) $[\mathrm{M}+\mathrm{Na}]^{+}$: calcd. for $\mathrm{C}_{18} \mathrm{H}_{22} \mathrm{O}_{5} \mathrm{Na} 341.1365$, found 341.1329. $[\alpha]_{\mathrm{D}}^{23}=19^{\circ}\left(\mathrm{c}=1.0 \mathrm{CHCl}_{3}\right)$.

Fernandez-Forner, F. Albericia, Tetrahedron Lett. 2005, 46, 7271-7174. 


\section{General procedure for conversion of aryl- $C$-glycoside bis-acetates to}

\section{bis-carbonates $8 b$}

\section{Table SI-3:}
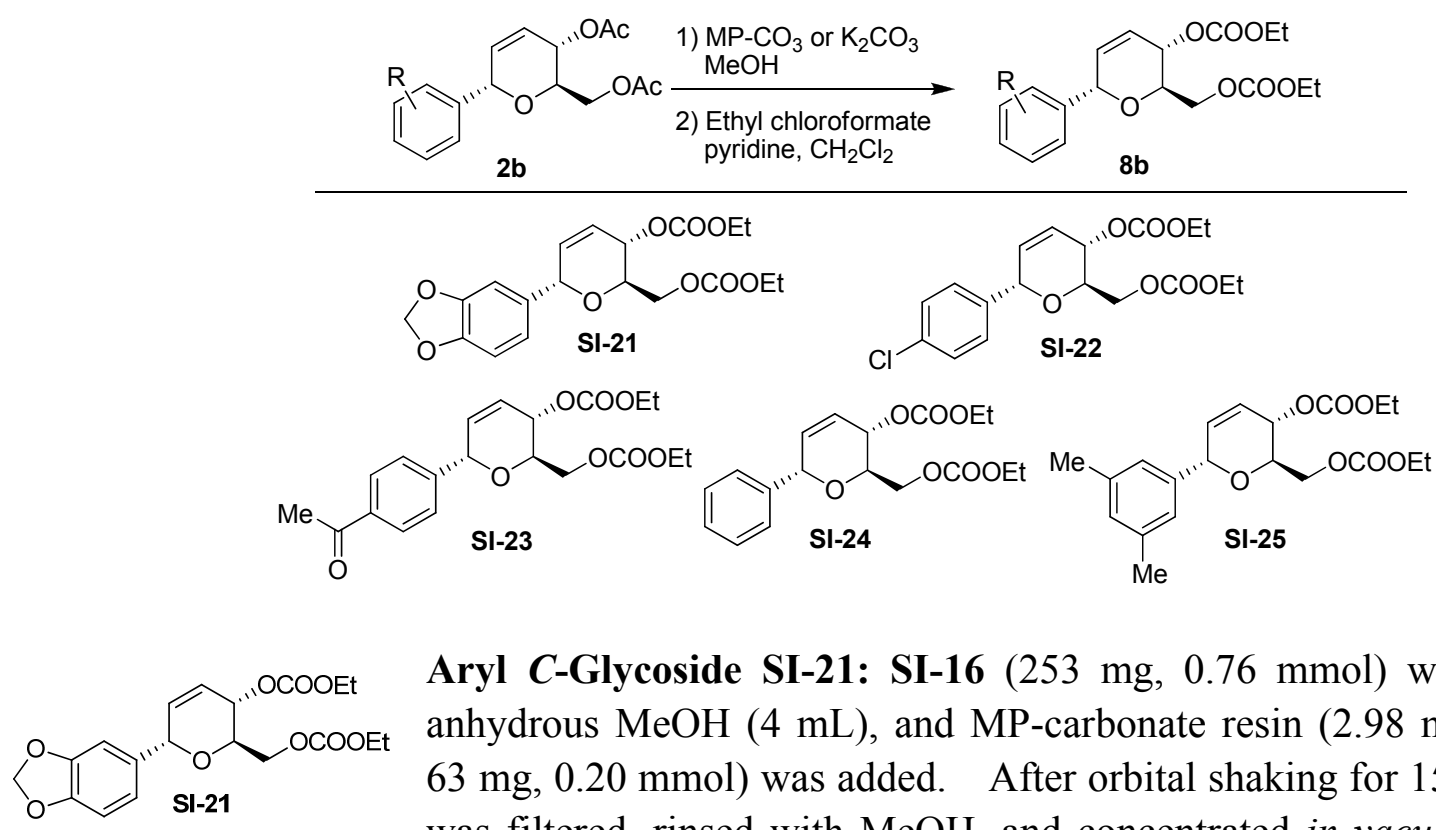

Aryl $\boldsymbol{C}$-Glycoside SI-21: SI-16 (253 $\mathrm{mg}, 0.76 \mathrm{mmol})$ was dissolved in anhydrous $\mathrm{MeOH}(4 \mathrm{~mL})$, and MP-carbonate resin $(2.98 \mathrm{mmol} / \mathrm{g}$ loading, $63 \mathrm{mg}, 0.20 \mathrm{mmol}$ ) was added. After orbital shaking for $15 \mathrm{~h}$, the reaction was filtered, rinsed with $\mathrm{MeOH}$, and concentrated in vacuo to provide 15 $\mathrm{mg}$ of the crude diol, which was dissolved in $\mathrm{CH}_{2} \mathrm{Cl}_{2}(3 \mathrm{~mL})$ and cooled to $0^{\circ} \mathrm{C}$. Pyridine $(0.13 \mathrm{~mL}$, $1.6 \mathrm{mmol})$ and ethyl chloroformate $(0.15 \mathrm{~mL}, 1.6 \mathrm{mmol})$ were added. The reaction was warmed to room temperature, stirred for $12 \mathrm{~h}$, diluted with sat. aqueous sodium bicarbonate and extracted with $\mathrm{CH}_{2} \mathrm{Cl}_{2}$. The organic layer was washed with brine, dried, and concentrated. Chromatography over $\mathrm{SiO}_{2}\left(0-40 \%\right.$ ethyl acetate/pet ether) provided $\mathbf{S I - 2 1}\left(14 \mathrm{mg}, 68 \%\right.$ yield) as a viscous oil. ${ }^{1} \mathrm{H}$ NMR $\left(400 \mathrm{MHz}, \mathrm{CDCl}_{3}\right): \delta 6.88(\mathrm{~d}, 1 \mathrm{H}, J=1.2), 6.82(\mathrm{dd}, 1 \mathrm{H}, J=2.0,8.0), 6.76(\mathrm{~d}, 1 \mathrm{H}, J=8.0), 6.12$ (ddd, $1 \mathrm{H}, J=1.6,3.2,10.8), 6.03(\mathrm{dd}, 1 \mathrm{H}, J=2.0,10.4), 5.95(\mathrm{~m}, 2 \mathrm{H}), 5.22(\mathrm{~m}, 1 \mathrm{H}), 5.17(\mathrm{~m}, 1 \mathrm{H}), 4.29(\mathrm{dd}$, $1 \mathrm{H}, J=5.2,11.6), 4.21$ (q, $2 \mathrm{H}, J=7.2), 4.17$ (q, $2 \mathrm{H}, 6.8), 3.86(\mathrm{~m}, 1 \mathrm{H}), 1.31$ (t, $3 \mathrm{H}, J=7.6), 1.28(\mathrm{t}, 3 \mathrm{H}$, $J=6.8) \mathrm{ppm} ;{ }^{13} \mathrm{C}$ NMR $\left(100 \mathrm{MHz}, \mathrm{CDCl}_{3}\right): \delta 154.9,154.5,147.8,147.6,132.1,131.7,124.6,121.8$, 108.7, 107.9, 101.1, 73.6, 68.2, 68.2, 66.0, 64.4, 64.1, $14.1 \mathrm{ppm}$; IR (neat): 2986, 2901, 1740,1487, $1445,1371,1262,1041 \mathrm{~cm}^{-1} ;[\alpha]_{\mathrm{D}}^{23}=-2^{\mathrm{o}}\left(\mathrm{c}=1.0 \mathrm{CH}_{2} \mathrm{Cl}_{2}\right)$.

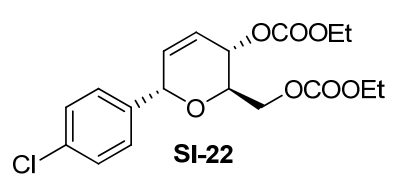

Aryl $\boldsymbol{C}$-Glycoside SI-22: SI-17 (201 $\mathrm{mg}, 0.62 \mathrm{mmol})$ was dissolved in anhydrous $\mathrm{MeOH}(2 \mathrm{~mL})$ and MP-carbonate resin $(2.98 \mathrm{mmol} / \mathrm{g}$ loading, $100 \mathrm{mg}, 0.30 \mathrm{mmol}$ ) was added. After orbital shaking for $5 \mathrm{~h}$, the reaction was filtered, rinsed with $\mathrm{MeOH}$, and concentrated in vacuo to provide 180 $\mathrm{mg}$ of the crude diol, which was dissolved in $\mathrm{CH}_{2} \mathrm{Cl}_{2}(3 \mathrm{~mL})$ and cooled to $0^{\circ} \mathrm{C}$. Pyridine $(0.16 \mathrm{~mL}$, $2.0 \mathrm{mmol})$ and ethyl chloroformate $(0.19 \mathrm{~mL}, 2.0 \mathrm{mmol})$ were added. The reaction was warmed to room temperature, stirred for $12 \mathrm{~h}$, diluted with sat. aqueous sodium bicarbonate and extracted with 
$\mathrm{CH}_{2} \mathrm{Cl}_{2}$. The organic layer was washed with brine, dried, and concentrated. Chromatography over $\mathrm{SiO}_{2}\left(0-40 \%\right.$ ethyl acetate/pet ether) provided SI-22 (108 mg, 42\% yield) as viscous oil. ${ }^{1} \mathrm{H}$ NMR $\left(400 \mathrm{MHz}, \mathrm{CDCl}_{3}\right): \delta 7.32(\mathrm{~m}, 4 \mathrm{H}), 6.16(\mathrm{ad}, 1 \mathrm{H}, J=8.8), 6.07$ (d, $\left.1 \mathrm{H}, J=10.4\right), 5.29$ (d, $\left.1 \mathrm{H}, J=2.0\right)$, $5.18(\mathrm{~m}, 1 \mathrm{H}), 4.29(\mathrm{dd}, 1 \mathrm{H}, J=5.6,12.0), 4.22(\mathrm{~m}, 5 \mathrm{H}), 3.86(\mathrm{~m}, 1 \mathrm{H}), 1.31(\mathrm{~m}, 6 \mathrm{H}) \mathrm{ppm} ;{ }^{13} \mathrm{C}$ NMR $\left(100 \mathrm{MHz}, \mathrm{CDCl}_{3}\right): \delta 155.0,154.5,136.9,134.2,131.3,129.4,128.6,124.9,73.0,68.7,68.0,65.9$, 64.4, 64.2, 14.2 ppm; IR (neat): 2983, 1748, 1495, 1374, 1262, 1099, 1013, $873 \mathrm{~cm}^{-1} ; \quad[\alpha]_{\mathrm{D}}^{23}=-22^{\circ}$ $\left(\mathrm{c}=1.0 \mathrm{CHCl}_{3}\right)$.

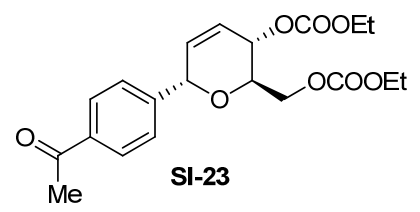

Aryl $\boldsymbol{C}$-Glycoside SI-23: SI-18 (108 $\mathrm{mg}, 0.54 \mathrm{mmol})$ was dissolved in anhydrous $\mathrm{MeOH}(3 \mathrm{~mL})$ and MP-carbonate resin $(2.98 \mathrm{mmol} / \mathrm{g}$ loading, 90 $\mathrm{mg}, 0.30 \mathrm{mmol}$ ) was added. After orbital shaking for $5 \mathrm{~h}$, the reaction was filtered, rinsed with $\mathrm{MeOH}$, and concentrated in vacuo to provide 12 $\mathrm{mg}$ of the crude diol, which was dissolved in $\mathrm{CH}_{2} \mathrm{Cl}_{2}(2 \mathrm{~mL})$ and cooled to $0^{\circ} \mathrm{C}$. Pyridine $(0.12 \mathrm{~mL}$, $1.4 \mathrm{mmol})$ and ethyl chloroformate $(0.14 \mathrm{~mL}, 1.4 \mathrm{mmol})$ were added. The reaction was warmed to room temperature, stirred for $12 \mathrm{~h}$, diluted with sodium bicarbonate, and extracted with $\mathrm{CH}_{2} \mathrm{Cl}_{2}$. The organic layer was washed with brine, dried, and concentrated. Chromatography over $\mathrm{SiO}_{2}$ (0-40\% ethyl acetate/pet ether) provided SI-23 (136 mg, 72\% yield) as viscous oil. ${ }^{1} \mathrm{H}$ NMR (400 $\left.\mathrm{MHz}, \mathrm{CDCl}_{3}\right): \delta 7.96(\mathrm{~d}, 2 \mathrm{H}, J=8.0), 7.50(\mathrm{~d}, 2 \mathrm{H}, J=8.8), 6.12(\mathrm{dt}, 1 \mathrm{H}, J=1.6,10.8), 6.09(\mathrm{dd}, 1 \mathrm{H}$, $J=1.6,10.4), 5.34$ (m, 1H), 5.19 (appd, $1 \mathrm{H}, J=5.2$ ), 4.33 (dd, $1 \mathrm{H}, J=5.6,11.6), 4.19$ (m, 6H), 3.79 (m, $1 \mathrm{H}), 2.59(\mathrm{~s}, 3 \mathrm{H}), 2.61(\mathrm{~s}, 3 \mathrm{H}), 1.31(\mathrm{~m}, 6 \mathrm{H}) \mathrm{ppm} ;{ }^{13} \mathrm{C} \mathrm{NMR}\left(100 \mathrm{MHz}, \mathrm{CDCl}_{3}\right): \delta$ 197.7, 155.0, 154.4, 143.7, 136.8, 131.1, 128.6, 127.9, 124.9, 73.1, 69.1, 67.9, 65.9, 64.5, 64.2, 26.7, 14.2, 14.1 ppm; IR (neat): $3440,2971,2920,1732,1717,1612,1390,1270,1072 \mathrm{~cm}^{-1} ;[\alpha]_{\mathrm{D}}^{23}=-9^{\circ}(\mathrm{c}=1.0$ $\mathrm{CH}_{2} \mathrm{Cl}_{2}$ ).

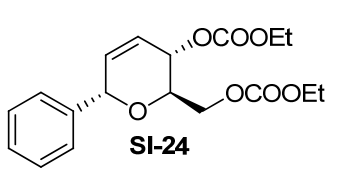

Aryl C-Glycoside SI-24: SI-19 (363 mg, $1.1 \mathrm{mmol}$ ) was dissolved in anhydrous $\mathrm{MeOH}(3 \mathrm{~mL})$ and MP-carbonate resin( $2.98 \mathrm{mmol} / \mathrm{g}$ loading, 200 $\mathrm{mg}, 0.50 \mathrm{mmol}$ ) was added. After orbital shaking for $5 \mathrm{~h}$, the reaction was filtered, rinsed with $\mathrm{MeOH}$ and concentrated in vacuo to provide $265 \mathrm{mg}$ of the crude diol, which was dissolved in $\mathrm{CH}_{2} \mathrm{Cl}_{2}(4 \mathrm{~mL})$ and cooled to $0^{\circ} \mathrm{C}$. Pyridine $(0.35 \mathrm{~mL}, 4.4 \mathrm{mmol})$ and ethyl chloroformate $(0.42 \mathrm{~mL}, 4.4 \mathrm{mmol})$ were added. The reaction was warmed to room temperature, stirred for $12 \mathrm{~h}$, diluted with sat. aqueous sodium bicarbonate, and extracted with $\mathrm{CH}_{2} \mathrm{Cl}_{2}$. The organic layer was washed with brine, dried, and concentrated. Chromatography over $\mathrm{SiO}_{2}\left(0-40 \%\right.$ ethyl acetate/pet ether) provided $\mathrm{SI}-24$ (136 mg, 46\% yield) as a viscous oil. ${ }^{1} \mathrm{H}$ NMR (400 MHz, $\mathrm{CDCl}_{3}$ ): 7.31-7.40 (m, 5H), 6.19 (ddd, $\left.1 \mathrm{H}, J=1.6,3.2,10.4\right), 6.06$ (dt, $1 \mathrm{H}, J=2.0,10.0$ ), $5.32(\mathrm{~m}, 1 \mathrm{H}), 5.20(\mathrm{~m}, 1 \mathrm{H}), 5.6(\mathrm{dd}, 1 \mathrm{H}, J=5.6,11.6), 4.19(\mathrm{~m}, 5 \mathrm{H}), 3.88(\mathrm{~m}, 1 \mathrm{H}), 1.31(\mathrm{t}, 1 \mathrm{H}, J=7.2)$, 1.28 (s, $1 \mathrm{H}, J=7.2) \mathrm{ppm} ;{ }^{13} \mathrm{C}$ NMR $\left(100 \mathrm{MHz}, \mathrm{CDCl}_{3}\right): \delta 154.9,154.5,138.3,131.8,128.4,128.2$, 128.0, 124.4, 73.7, 68.6, 68.2, 66.0, 64.3, 64.1, $14.1 \mathrm{ppm}$; IR (neat): 2986, 1744, 1456, 1378, 1251, $1107,1014,877 \mathrm{~cm}^{-1} ;[\alpha]_{\mathrm{D}}^{23}=-22^{\circ}\left(\mathrm{c}=1.0 \mathrm{CHCl}_{3}\right)$. 


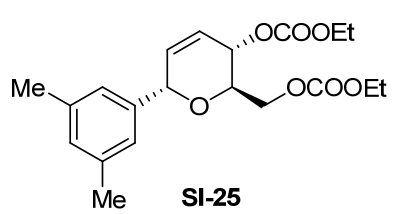

Aryl C-Glycoside SI-25: SI-20 (253 $\mathrm{mg}, 0.8 \mathrm{mmol})$ was dissolved in anhydrous $\mathrm{MeOH}(2 \mathrm{~mL})$ and $\mathrm{K}_{2} \mathrm{CO}_{3}(22 \mathrm{mg}, 0.16 \mathrm{mmol})$ was added. After stirring for $2 \mathrm{~h}$, the reaction was filtered, rinsed with $\mathrm{MeOH}$ and concentrated in vacuo to provide $186 \mathrm{mg}$ of the crude diol, which was dissolved in $\mathrm{CH}_{2} \mathrm{Cl}_{2}(3 \mathrm{~mL})$ and cooled to $0^{\circ} \mathrm{C}$. Pyridine $(0.26 \mathrm{~mL}, 3.2$ mmol) and ethyl chloroformate $(0.30 \mathrm{~mL}, 3.2 \mathrm{mmol})$ were added. The reaction was warmed to room temperature, stirred for $2 \mathrm{~h}$, diluted with sat. aqueous sodium bicarbonate, and extracted with $\mathrm{CH}_{2} \mathrm{Cl}_{2}$. The organic layer was washed with brine, dried, and concentrated. Chromatography over $\mathrm{SiO}_{2}$ (0-40\% ethyl acetate/pet ether) provided SI-25 (218 mg, 73\% yield) as a viscous oil. ${ }^{1} \mathrm{H}$ NMR $\left(400 \mathrm{MHz}, \mathrm{CDCl}_{3}\right): 7.00(\mathrm{~s}, 2 \mathrm{H}), 6.95(\mathrm{~s}, 1 \mathrm{H}), 6.17(\mathrm{dq}, 1 \mathrm{H}, J=1.6,3.2,10.8), 5.04$ (dt, 1H, $J=2.4$, 10.8), 5.20 (m, 2H), 4.31 (dd, 1H, $J=5.6,11.6), 4.22(\mathrm{~m}, 5 \mathrm{H}), 3.90(\mathrm{~m}, 1 \mathrm{H}), 2.31(\mathrm{~s}, 6 \mathrm{H}), 1.31(\mathrm{~m}, 6 \mathrm{H})$ ppm; ${ }^{13} \mathrm{C}$ NMR $\left(100 \mathrm{MHz}, \mathrm{CDCl}_{3}\right): \delta 154.9,154.5,138.1,137.9,131.9,129.8,125.8,124.3,73.8$, 68.5, 68.3, 66.0, 64.3, 64.1, 21.2, 14.1 ppm; IR (neat): 2979, 1744, 1612, 1452, 1379, 1270, 1014, $881 \mathrm{~cm}^{-1} ;[\alpha]_{\mathrm{D}}^{23}=5^{\circ}\left(\mathrm{c}=1.0 \mathrm{CHCl}_{3}\right)$.

\section{General procedure for conversion of alkynyl-C-glycoside bis-acetates}

\section{to bis-carbonates, Table SI-4:}
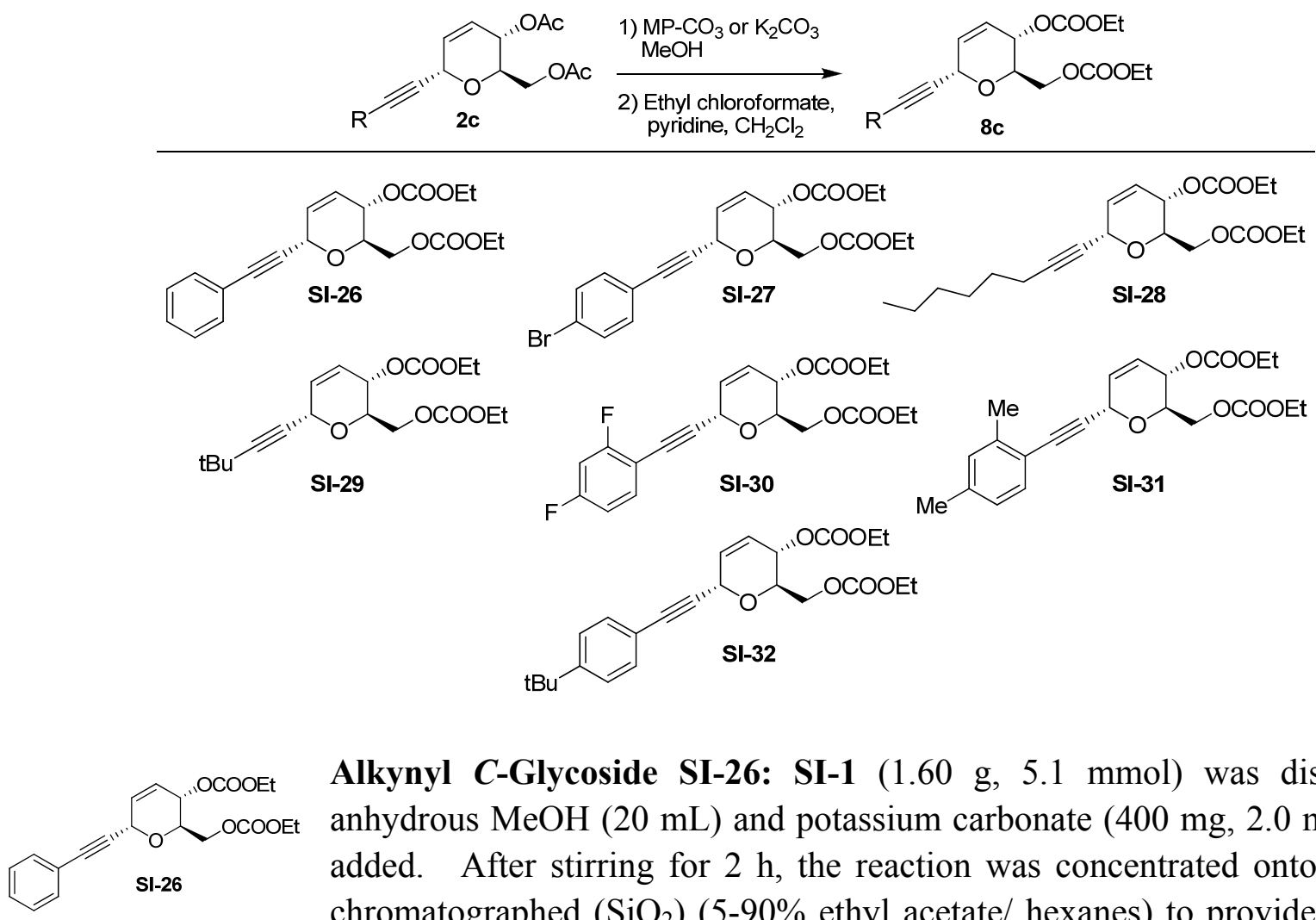

Alkynyl C-Glycoside SI-26: SI-1 (1.60 g, $5.1 \mathrm{mmol})$ was dissolved in anhydrous $\mathrm{MeOH}(20 \mathrm{~mL})$ and potassium carbonate $(400 \mathrm{mg}, 2.0 \mathrm{mmol})$ was added. After stirring for $2 \mathrm{~h}$, the reaction was concentrated onto $\mathrm{SiO}_{2}$ and chromatographed $\left(\mathrm{SiO}_{2}\right)$ (5-90\% ethyl acetate/ hexanes) to provide $1.17 \mathrm{~g}$ of the diol, which was dissolved in $\mathrm{CH}_{2} \mathrm{Cl}_{2}(7 \mathrm{~mL})$ and cooled to $0^{\circ} \mathrm{C}$. Pyridine $(1.50 \mathrm{~mL}, 19.0 \mathrm{mmol})$ and ethyl chloroformate $(1.40 \mathrm{~mL}, 15.0 \mathrm{mmol})$ were added consecutively. The reaction was 
warmed to room temperature, stirred for $3 \mathrm{~h}$, diluted ethyl acetate, washed with sodium bicarbonate and brine, dried, and concentrated. Chromatography over $\mathrm{SiO}_{2}(0-40 \%$ ethyl acetate/pet ether) provided SI-26 (1.44 g, 99\% yield) as a viscous oil. ${ }^{1} \mathrm{H}$ NMR $\left(400 \mathrm{MHz}, \mathrm{CDCl}_{3}\right): \delta 7.43(\mathrm{~m}, 2 \mathrm{H})$, 7.32 (m, 3H), 5.99 (ddd, $1 \mathrm{H}, J=1.6,3.6,10.4), 5.89$ (dt, $1 \mathrm{H}, J=2.0,10.0), 5.22$ (dq, $1 \mathrm{H}, J=2.0,8.8$ ), $5.18(\mathrm{~m}, 1 \mathrm{H}), 4.39$ (dd, 1H, $J=2.8,11.6), 4.34$ (dd, $1 \mathrm{H}, J=4.8,11.6), 4.21(\mathrm{~m}, 4 \mathrm{H}), 1.32(\mathrm{t}, 1 \mathrm{H}$, $J=7.6), 1.28(\mathrm{t}, 1 \mathrm{H}, J=7.2) \mathrm{ppm} ;{ }^{13} \mathrm{C} \mathrm{NMR}\left(100 \mathrm{MHz}, \mathrm{CDCl}_{3}\right): \delta 154.9,154.3,131.8,129.6,128.6$, 128.2, 124.7, 122.0, 86.7, 84.3, 69.4, 68.0, 66.0, 64.4, 64.3, 64.1, 14.1 ppm; IR (neat): 2967, 1740, 1456, 1371, 1254, $1017 \mathrm{~cm}^{-1}$; HRMS (Tof) $[\mathrm{M}+\mathrm{Na}]^{+}$: calcd. for $\mathrm{C}_{20} \mathrm{H}_{22} \mathrm{O}_{7} \mathrm{Na}$ 397.1263, found 397.1233. $[\alpha]_{\mathrm{D}}^{23}=-56^{\circ}\left(\mathrm{c}=1.0 \mathrm{CHCl}_{3}\right)$.

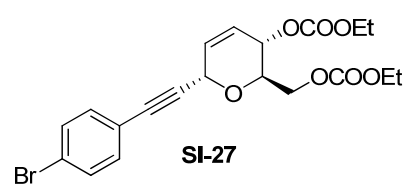

Alkynyl $\boldsymbol{C}$-Glycoside SI-27: Diacetate SI-5 $(38 \mathrm{mg}, 0.97 \mathrm{mmol}$ ) was dissolved in anhydrous $\mathrm{MeOH}(3 \mathrm{~mL})$ and MP-carbonate resin $(2.98$ $\mathrm{mmol} / \mathrm{g}$ loading, $200 \mathrm{mg}, 0.5 \mathrm{mmol}$ ) was added. After orbital shaking for $15 \mathrm{~h}$, the reaction was filtered, rinsed with $\mathrm{MeOH}$, and concentrated to provide $304 \mathrm{mg}$ of the diol, which was dissolved in $\mathrm{CH}_{2} \mathrm{Cl}_{2}(4 \mathrm{~mL})$ and cooled to $0^{\circ} \mathrm{C}$. Pyridine $(0.320 \mathrm{~mL}, 3.9 \mathrm{mmol})$ and ethyl chloroformate $(0.32 \mathrm{~mL}, 3.9 \mathrm{mmol})$ were added. The reaction was warmed to room temperature, stirred for $15 \mathrm{~h}$, diluted methylene chloride, washed with sat. aqeous sodium bicarbonate and brine, dried, and concentrated. Chromatography over $\mathrm{SiO}_{2}(0-45 \%$ ethyl acetate/pet ether) provided SI-27 (175 mg, 39\% yield) as a viscous oil. ${ }^{1} \mathrm{H}$ NMR (400 MHz, $\left.\mathrm{CDCl}_{3}\right): \delta 7.45(\mathrm{~d}, 2 \mathrm{H}, J=8.4), 7.29$ (d, $\left.2 \mathrm{H}, J=8.8\right), 5.98$ (ddd, $\left.1 \mathrm{H}, J=1.6,3.2,10.0\right), 5.90(\mathrm{dt}, 1 \mathrm{H}$, $J=2.0,10.4), 5.21$ (dq, $1 \mathrm{H}, J=2.0,24.4), 5.17(\mathrm{~m}, 1 \mathrm{H}), 4.39$ (dd, $1 \mathrm{H}, J=3.2,12.0), 4.33$ (dd, $1 \mathrm{H}$, $J=4.4,11.6), 4.21$ (q, $2 \mathrm{H}, J=7.2), 4.20$ (q, $2 \mathrm{H}, J=7.2), 1.32$ (t, $3 \mathrm{H}, J=7.2), 1.29$ (t, $3 \mathrm{H}, J=7.6) \mathrm{ppm}$; ${ }^{13} \mathrm{C}$ NMR $\left(100 \mathrm{MHz}, \mathrm{CDCl}_{3}\right): \delta 155.0,154.4,133.3,131.6,129.3,125.0,123.1,121.0,85.7,85.5$, 69.6, 67.9, 66.1, 64.5, 64.3, 64.3, 14.2 ppm; IR (neat): 3398, 2974, 1748, 1378, 1247, 1013, $889 \mathrm{~cm}^{-1}$; HRMS (Tof) $[\mathrm{M}+\mathrm{Na}]^{+}$: calcd. for $\mathrm{C}_{20} \mathrm{H}_{21} \mathrm{O}_{7} \mathrm{NaBr} 475.0368$, found $475.0371 .[\alpha]_{\mathrm{D}}{ }^{23}=-75^{\circ}(\mathrm{c}=1.0$ $\left.\mathrm{CHCl}_{3}\right)$.

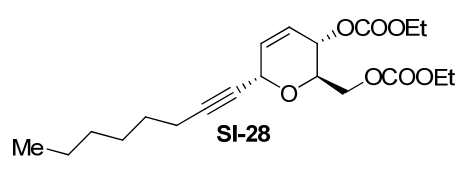

Alkynyl C-Glycoside SI-28: Diacetate SI-7 (357 mg, $1.11 \mathrm{mmol}$ ) was dissolved in anhydrous $\mathrm{MeOH}(5 \mathrm{~mL})$ and MP-carbonate resin(2.98 $\mathrm{mmol} / \mathrm{g}$ loading, $200 \mathrm{mg}, 0.5 \mathrm{mmol}$ ) was added. After orbital shaking for $15 \mathrm{~h}$, the reaction filtered, rinsed with $\mathrm{MeOH}$, and concentrated to provide $241 \mathrm{mg}$ diol, which was dissolved in $\mathrm{CH}_{2} \mathrm{Cl}_{2}(3 \mathrm{~mL})$ and cooled to $0^{\circ} \mathrm{C}$. Pyridine $(0.327$ $\mathrm{mL}, 4.0 \mathrm{mmol})$ and ethyl chloroformate $(0.387 \mathrm{~mL}, 4.0 \mathrm{mmol})$ were added. The reaction was warmed to room temperature, stirred for $15 \mathrm{~h}$, diluted with $\mathrm{CH}_{2} \mathrm{Cl}_{2}$, washed with sat. aqueous sodium bicarbonate and brine, dried, and concentrated. Chromatography over $\mathrm{SiO}_{2}(0-40 \%$ ethyl acetate/pet ether) provided SI-28 (324 mg, 39\% yield) as a viscous oil. ${ }^{1} \mathrm{H}$ NMR (400 MHz, $\mathrm{CDCl}_{3}$ ): $\delta 5.82(\mathrm{ddd}, 1 \mathrm{H}, J=2.0,3.4,10.3$ ), $5.73(\mathrm{dt}, 1 \mathrm{H}, J=1.6,10.4), 5.08$ (dq, $1 \mathrm{H}, J=2.0,9.0), 4.86$ $(\mathrm{m}, 1 \mathrm{H}), 5.08(\mathrm{~m}, 1 \mathrm{H}), 4.85(\mathrm{~m}, 1 \mathrm{H}), 4.17(\mathrm{~m}, 7 \mathrm{H}), 2.12(\mathrm{td}, 2 \mathrm{H}, J=2.0,7.2), 1.40(\mathrm{~m}, 2 \mathrm{H}), 1.22(\mathrm{~m}$, $12 \mathrm{H}), 0.81(\mathrm{t}, 3 \mathrm{H}, 6 J=6.8) \mathrm{ppm} ;{ }^{13} \mathrm{C}$ NMR $\left(100 \mathrm{MHz}, \mathrm{CDCl}_{3}\right): \delta 154.8,152.2,130.3,123.8,87.8$, $75.3,68.9,67.9,66.0,64.2,64.0,63.9,31.1,28.2,28.2,22.3,18.5,14.0,13.8$ ppm; IR (neat): 3452 , 
2963, 2939, 2854, 2361, 2341, 1740, 1464, 1371, 1266, 1018, $878 \mathrm{~cm}^{-1} ;[\alpha]_{\mathrm{D}}^{23}=-29^{\circ}(\mathrm{c}=1.0$ $\left.\mathrm{CHCl}_{3}\right)$.

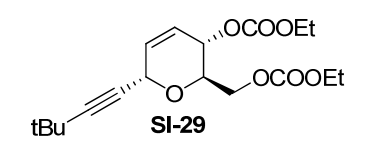

Alkynyl C-Glycoside SI-29: Diacetate SI-4 (150 mg, $0.51 \mathrm{mmol})$ was dissolved in anhydrous $\mathrm{MeOH}(2 \mathrm{~mL})$ and $\mathrm{MP}$-carbonate resin $(2.98 \mathrm{mmol} / \mathrm{g}$ loading, $80 \mathrm{mg}, 0.2 \mathrm{mmol}$ ) was added. After orbital shaking for $15 \mathrm{~h}$, the reaction was filtered, rinsed with $\mathrm{MeOH}$, and concentrated to provide $113 \mathrm{mg}$ of the diol, which was dissolved in $\mathrm{CH}_{2} \mathrm{Cl}_{2}(2 \mathrm{~mL})$ and cooled to $0^{\circ} \mathrm{C}$. Pyridine $(0.170 \mathrm{~mL}, 2.1 \mathrm{mmol})$ and ethyl chloroformate $(0.200 \mathrm{~mL}, 2.1 \mathrm{mmol})$ were added. The reaction was warmed to room temperature, stirred for $4 \mathrm{~h}$, diluted with $\mathrm{CH}_{2} \mathrm{Cl}_{2}$, washed with sat. aqeous sodium bicarbonate and brine, dried, and concentrated. Chromatography over $\mathrm{SiO}_{2}(0-40 \%$ ethyl acetate/pet ether) provided SI-29 (324 mg, $41 \%$ yield) as a viscous oil. ${ }^{1} \mathrm{H}$ NMR $\left(400 \mathrm{MHz}, \mathrm{CDCl}_{3}\right): \delta 5.84(\mathrm{~m}, 1 \mathrm{H}), 5.80(\mathrm{~m}, 1 \mathrm{H}), 5.15(\mathrm{~m}, 1 \mathrm{H})$, $4.93(\mathrm{~m}, 1 \mathrm{H}), 4.10-4.37(\mathrm{~m}, 7 \mathrm{H}), 1.31(\mathrm{~m}, 6 \mathrm{H}), 1.19(\mathrm{~s}, 9 \mathrm{H}) \mathrm{ppm} ;{ }^{13} \mathrm{C} \mathrm{NMR}\left(100 \mathrm{MHz}, \mathrm{CDCl}_{3}\right): \delta$ 155.0, 154.4, 130.5, 124.0, 95.9, 73.9, 69.0, 68.2, 66.1, 64.4, 64.2, 64.1, 30.7 27.4, 14.2 ppm; IR (neat): $3425,2974,1744,1378,1231,1049 \mathrm{~cm}^{-1} ;[\alpha]_{\mathrm{D}}^{23}=+23.0^{\circ}\left(\mathrm{c}=1.0 \mathrm{CH}_{2} \mathrm{Cl}_{2}\right)$.

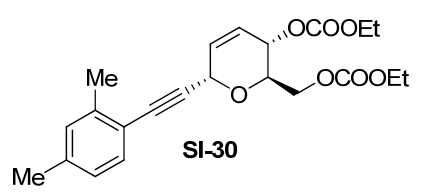

Alkynyl $\boldsymbol{C}$-Glycoside SI-30: Diacetate SI-10 (540 mg, $1.60 \mathrm{mmol})$ was dissolved in anhydrous $\mathrm{MeOH}(2 \mathrm{~mL})$ and $\mathrm{MP}$-carbonate resin $(2.98 \mathrm{mmol} / \mathrm{g}$ loading, $100 \mathrm{mg}, 0.32 \mathrm{mmol}$ ) was added. After orbital shaking for $2 \mathrm{~h}$, the reaction was filtered, rinsed with $\mathrm{MeOH}$, and concentrated to provide 355 $\mathrm{mg}$ of the diol, which was dissolved in $\mathrm{CH}_{2} \mathrm{Cl}_{2}(2 \mathrm{~mL})$ and cooled to $0^{\circ} \mathrm{C}$. Pyridine $(0.445 \mathrm{~mL}, 5.5$ $\mathrm{mmol})$ and ethyl chloroformate $(0.526 \mathrm{~mL}, 5.5 \mathrm{mmol})$ were added. The reaction was warmed to room temperature, stirred for $4 \mathrm{~h}$, diluted with $\mathrm{CH}_{2} \mathrm{Cl}_{2}$, washed with sat. aqeous sodium bicarbonate and brine, dried, and concentrated. Chromatography over $\mathrm{SiO}_{2}(0-40 \%$ ethyl acetate/pet ether) provided SI-30 (458 mg, 83\% yield) as a viscous oil. ${ }^{1} \mathrm{H}$ NMR (400 $\left.\mathrm{MHz}, \mathrm{CDCl}_{3}\right): \delta 7.21(\mathrm{~s}, 1 \mathrm{H})$, $7.03(\mathrm{~m}, 2 \mathrm{H}), 6.00(\mathrm{dt}, 1 \mathrm{H}, J=1.6,10.0), 5.89(\mathrm{~m}, 1 \mathrm{H}), 5.22,(\mathrm{~m}, 1 \mathrm{H}), 4.35(\mathrm{~m}, 1 \mathrm{H}), 4.23(\mathrm{~m}, 5 \mathrm{H}), 2.35$ $(\mathrm{s}, 3 \mathrm{H}), 2.27(\mathrm{~s}, 3 \mathrm{H}), 1.30(\mathrm{~m}, 6 \mathrm{H}) \mathrm{ppm} ;{ }^{13} \mathrm{C} \mathrm{NMR}\left(100 \mathrm{MHz}, \mathrm{CDCl}_{3}\right): \delta 155.0,154.4,137.3,134.9$, $132.5,129.7,129.5,129.2,124.7,121.6,87.8,86.0,69.5,68.1,66.1,64.5,64.4,64.1,20.6,20.0,14.1$ ppm; IR (neat): 2994, 1748, 1456, 1371, 1258, $1314 \mathrm{~cm}^{-1} ;[\alpha]_{\mathrm{D}}^{23}=-56^{\mathrm{o}}\left(\mathrm{c}=1.0 \mathrm{CH}_{2} \mathrm{Cl}_{2}\right)$.

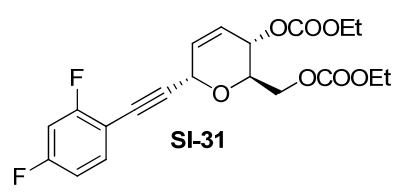

Alkynyl C-Glycoside SI-31: Diacetate SI-11 (298 $\mathrm{mg}, 0.85 \mathrm{mmol})$ was dissolved in anhydrous $\mathrm{MeOH}(2 \mathrm{~mL})$ and $\mathrm{MP}$-carbonate resin $(2.98 \mathrm{mmol} / \mathrm{g}$ loading, $43 \mathrm{mg}, 0.13 \mathrm{mmol}$ ) was added. After orbital shaking for $3 \mathrm{~h}$, the reaction was filtered, rinsed with $\mathrm{MeOH}$, and concentrated to provide 238

$\mathrm{mg}$ of the diol, which was dissolved in $\mathrm{CH}_{2} \mathrm{Cl}_{2}(2 \mathrm{~mL})$ and cooled to $0^{\circ} \mathrm{C}$. Pyridine $(0.274 \mathrm{~mL}, 3.4$ $\mathrm{mmol})$ and ethyl chloroformate $(0.324 \mathrm{~mL}, 3.4 \mathrm{mmol})$ were added. The reaction was warmed to room temperature, stirred for $2 \mathrm{~h}$, diluted with $\mathrm{CH}_{2} \mathrm{Cl}_{2}$, washed with sat. aqeous sodium bicarbonate and brine, dried, and concentrated. Chromatography over $\mathrm{SiO}_{2}(0-40 \%$ ethyl acetate/pet ether) provided SI-31 $\left(275 \mathrm{mg}, 79 \%\right.$ yield) as a viscous oil. ${ }^{1} \mathrm{H}$ NMR $\left(400 \mathrm{MHz}, \mathrm{CDCl}_{3}\right): \delta 7.40(\mathrm{~m}, 1 \mathrm{H})$, $6.83(\mathrm{~m}, 2 \mathrm{H}), 5.99(\mathrm{dq}, 1 \mathrm{H}, J=1.6,3.2,10.4), 5.92(\mathrm{dt}, 1 \mathrm{H}, J=1.6,3.6), 5.21(\mathrm{~m}, 2 \mathrm{H}), 4.39$ (dd, $1 \mathrm{H}$, 
$J=3.2,12.0), 4.34(\mathrm{dd}, 1 \mathrm{H}, J=4.8,7.6), 4.22(\mathrm{~m}, 5 \mathrm{H}), 1.30(\mathrm{~m}, 6 \mathrm{H}) \mathrm{ppm} ;{ }^{13} \mathrm{C} \mathrm{NMR}\left(100 \mathrm{MHz}, \mathrm{CDCl}_{3}\right)$ : $\delta 164.3(\mathrm{dd}, 1 \mathrm{C}, J=46.8,131.6), 161.8(\mathrm{dd}, 1 \mathrm{C}, J=46.8,122.8), 155.0,154.4,134.4(\mathrm{dd}, 1 \mathrm{C}, J=8.8$, 38.4), 129.1, 125.2, 111.5 (dd, $1 \mathrm{C}, J=14.8,85.2$ ), 104.3 (dd, $1 \mathrm{C}, J=96.8,102.8$ ), 89.4, 69.6, 67.9, 66.1, 64.5, 64.3, 64.2, 14.2, 14.1 ppm; IR (neat): 2986, 1744, 1619, 1495, 1254, 1153, 1091, $1014 \mathrm{~cm}^{-1}$; HRMS (Tof) $[\mathrm{M}+\mathrm{Na}]^{+}$: calcd. for $\mathrm{C}_{20} \mathrm{H}_{20} \mathrm{O}_{7} \mathrm{NaF}_{2} 433.1075$, found 433.1023. $[\alpha]_{\mathrm{D}}^{23}=-67^{\circ}(\mathrm{c}=1.0$ $\mathrm{CH}_{2} \mathrm{Cl}_{2}$ ).

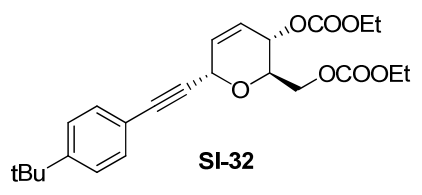

Alkynyl $\boldsymbol{C}$-Glycoside SI-32: Diacetate SI-12 (265 mg, $0.71 \mathrm{mmol})$ was dissolved in anhydrous $\mathrm{MeOH}(2 \mathrm{~mL})$ and $\mathrm{MP}$-carbonate resin (2.98 $\mathrm{mmol} / \mathrm{g}$ loading, $36 \mathrm{mg}, 0.1 \mathrm{mmol}$ ) was added. After orbital shaking for $3 \mathrm{~h}$, the reaction was filtered, rinsed with $\mathrm{MeOH}$, and concentrated to provide $177 \mathrm{mg}$ of the diol, which was dissolved in $\mathrm{CH}_{2} \mathrm{Cl}_{2}(2 \mathrm{~mL})$ and cooled to $0^{\circ} \mathrm{C}$. Pyridine $(0.188 \mathrm{~mL}, 2.3 \mathrm{mmol})$ and ethyl chloroformate $(0.222 \mathrm{~mL}, 2.3 \mathrm{mmol})$ were added. The reaction was warmed to room temperature, stirred for $15 \mathrm{~h}$, diluted with $\mathrm{CH}_{2} \mathrm{Cl}_{2}$, washed with sat. aqeous sodium bicarbonate and brine, dried, and concentrated. Chromatography over $\mathrm{SiO}_{2}(0-40 \%$ ethyl acetate/pet ether) provided SI-32 $\left(222.4 \mathrm{mg}, 89 \%\right.$ yield) as a viscous oil. ${ }^{1} \mathrm{H}$ NMR $\left(400 \mathrm{MHz}, \mathrm{CDCl}_{3}\right): \delta 7.36(\mathrm{~d}$, $1 \mathrm{H}, J=8.4$ ), 7.33 (d, 1H, $J=6.4$ ), 5.99 (dq, $1 \mathrm{H}, J=2.0,3.6,10.0), 5.89$ (dt, 1H, 2.0, 3.6), 5.23 (dq, $1 \mathrm{H}$, $J=2.0,4.0,9.2), 5.18(\mathrm{~m}, 1 \mathrm{H}), 4.39(\mathrm{dd}, 1 \mathrm{H}, J=2.8,12.0), 4.34(\mathrm{dd}, 1 \mathrm{H}, J=5.2,12.0), 4.23(\mathrm{~m}, 5 \mathrm{H})$, $1.31(\mathrm{~m}, 6 \mathrm{H}) \mathrm{ppm} ;{ }^{13} \mathrm{C} \mathrm{NMR}\left(100 \mathrm{MHz}, \mathrm{CDCl}_{3}\right): \delta$ 155.0, 154.4, 152.0, 131.6, 129.8, 131.7, 129.8, $125.2,124.6,119.0,86.9,83.7,69.4,68.1,66.1,64.5,64.2,34.7,31.1,14.2$ ppm; IR (neat): 2967, $1748,1371,1266,1009,784 \mathrm{~cm}^{-1} ; \quad[\alpha]_{\mathrm{D}}^{23}=-48^{\mathrm{o}}\left(\mathrm{c}=1.0 \mathrm{CH}_{2} \mathrm{Cl}_{2}\right)$.

\section{Phenol addition to C-glycosides-preliminary rehearsal screen}

Table SI- 5: Evaluation of different phenols for allylic addition.
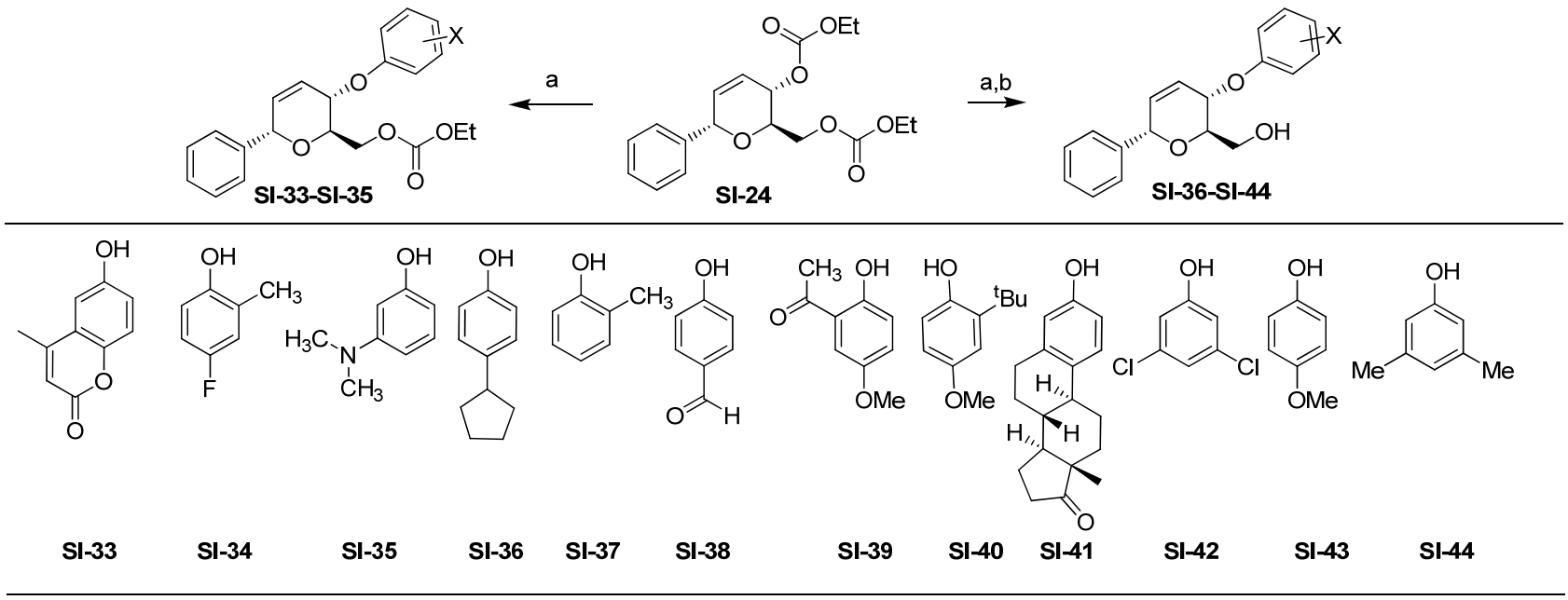

a) $\mathrm{Pd}_{2}(\mathrm{dba})_{3}-\mathrm{CHCl}_{3},(\mathrm{~S}, \mathrm{~S})$-Trost Ligand, phenol, $\mathrm{CH}_{2} \mathrm{Cl}_{2}$, $\mu$ wave $\left(100^{\circ} \mathrm{C}, 300 \mathrm{~W}\right), 15$ min. b) $\mathrm{K}_{2} \mathrm{CO}_{3}, \mathrm{MeOH}$

General Procedure for phenol addition. (Aryl ethers SI-36-SI-44). Dicarbonate SI-24 (540 mg, $1.5 \mathrm{mmol}),(1 S, 2 S)-(-)-$ 1,2- Diaminocyclohexane- N,N'-bis(2'-diphenylphosphinobenzoyl) 
(110 $\mathrm{mg}, 0.15 \mathrm{mmol}$ ), and Tris(dibenzylideneacetone) dipalladium(0)- chloroform adduct (80 $\mathrm{mg}$, $0.08 \mathrm{mmol})$ were dissolved in degassed $\mathrm{CH}_{2} \mathrm{Cl}_{2}(8.5 \mathrm{~mL})$ and stirred for $15 \mathrm{~min}$. The color of the solution changed from maroon to yellowish-orange. The solution $(0.500 \mathrm{~mL}, 0.09 \mathrm{mmol})$ was then transferred to separate $10 \mathrm{~mL}$ microwave vials containing the appropriate phenol $(0.14 \mathrm{mmol})$. Each reaction was irradiated for $15 \mathrm{~min}\left(150-300 \mathrm{~W}\right.$, Powermax enabled) at $100^{\circ} \mathrm{C}$. The solutions were then transferred to $20 \mathrm{~mL}$ scintillation vials and concentrated in vacuo. Compounds SI-33-SI-35 were purified by $\mathrm{SiO}_{2}$ chromatography $(0-40 \%$ ethyl acetate / hexanes) to provide the carbonate- protected aryl ether. For (SI-36-SI-44) the crude reaction mixtures were resuspended in $\mathrm{MeOH}(1.5 \mathrm{~mL})$ and $\mathrm{MP}-\mathrm{CO}_{3}$ or $\mathrm{K}_{2} \mathrm{CO}_{3}(0.25 \mathrm{eq})$ was added. After shaking for $12 \mathrm{~h}$, the reactions were filtered and concentrated. Purification by $\mathrm{SiO}_{2}$ chromatography $(0-80 \%$ ethyl acetate / hexanes) provided the desired aryl ethers SI-36-SI-44 in the yields described below.

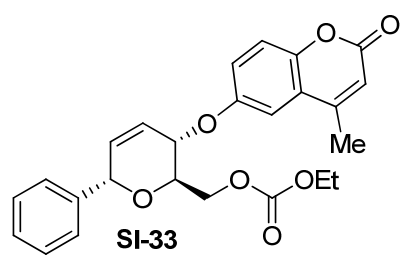

Aryl ether SI-33: Yield: $14.7(0.04 \mathrm{mmol}, 50 \%)$ as a film. ${ }^{1} \mathrm{H}$ NMR (400 $\left.\mathrm{MHz}, \mathrm{CDCl}_{3}\right): \delta 7.40-7.53(\mathrm{~m}, 6 \mathrm{H}), 6.95$ (appd, $\left.1 \mathrm{H}, J=2.4\right), 6.87(\mathrm{~m}, 2 \mathrm{H})$, 6.23 (ddd, 1H, 2.0, 3.2, 10.8), 6.17 (appd, $2 \mathrm{H}, J=4.8$ ), 5.41 (d, $1 \mathrm{H}, J=2.4$ ), 4.98 (dd, $1 \mathrm{H}, J=2.0,8.0), 4.32$ (d, 2H, $J=4.4), 4.15$ (q, $2 \mathrm{H}, J=6.8), 3.95$ (m, $1 \mathrm{H}), 2.41(\mathrm{~s}, 3 \mathrm{H}), 1.27$ (t, $1 \mathrm{H}, J=9.6) \mathrm{ppm} ;{ }^{13} \mathrm{C} \mathrm{NMR}\left(100 \mathrm{MHz}, \mathrm{CDCl}_{3}\right): \delta$ 214.0, 160.1, 155.0, 152.5, 138.5, 131.6, 128.6, 128.3, 127.9, 125.9, 124.3, 114.2, 113.1, 112.3, 103.4, 102.7, 74.3, 68.8, 68.7, 66.2, 64.3, 18.7, 14.2 ppm; IR (neat): 3378, 1957, 1739, 1608, 1440, 1386, $1266,1141,1068,1014 \mathrm{~cm}^{-1} ;[\alpha]_{\mathrm{D}}^{23}=+75^{\circ}\left(\mathrm{c}=1.0 \mathrm{CH}_{2} \mathrm{Cl}_{2}\right)$.

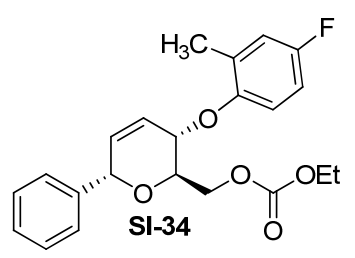

Aryl ether SI-34: Yield: $23 \mathrm{mg}(0.07 \mathrm{mmol}, 78 \%)$ as a film. ${ }^{1} \mathrm{H}$ NMR $(400$ $\left.\mathrm{MHz}, \mathrm{CDCl}_{3}\right): \delta$ 7.34-7.45 (m, 5H), $6.83(\mathrm{~m}, 3 \mathrm{H}), 6.19(\mathrm{dd}, 1 \mathrm{H}, J=1.2,2.8)$, 6.15 (dd, $1 \mathrm{H}, J=1.6,3.6), 5.39$ (d, $1 \mathrm{H}, J=1.6), 4.81$ (dd, $1 \mathrm{H}, J=2.0,8.8), 4.33$ (d, 2H, $J=3.6), 4.15$ (q, $2 \mathrm{H}, J=7.2), 3.94(\mathrm{~m}, 1 \mathrm{H}), 2.17$ (s, 3H), 1.27 (t, 3H, $J=7.2) \mathrm{ppm} ;{ }^{13} \mathrm{C}$ NMR $\left(100 \mathrm{MHz}, \mathrm{CDCl}_{3}\right): \delta 156.0,155.0,151.1,138.9,130.6$, $129.8,129.7,128.5,128.1,127.8,117.8,113.7,112.6,74.2,69.3,69.1,66.6,64.1,16.5,14.2 \mathrm{ppm}$; IR (neat): $3441,2963,2920,1748,1495,1456,1386,1262,1204,1060 \mathrm{~cm}^{-1} ;[\alpha]_{\mathrm{D}}{ }^{23}=+43^{\circ}(\mathrm{c}=1.0$ $\mathrm{CH}_{2} \mathrm{Cl}_{2}$ ).

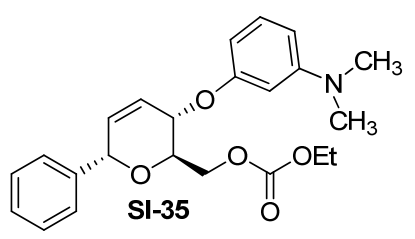

Aryl ether SI-35: Yield: $27 \mathrm{mg}(0.12 \mathrm{mmol}, 88 \%)$ as a film. ${ }^{1} \mathrm{H}$ NMR $(400$ $\left.\mathrm{MHz}, \mathrm{CDCl}_{3}\right): \delta 7.33-7.45(\mathrm{~m}, 5 \mathrm{H}), 7.15(\mathrm{t}, 1 \mathrm{H}, J=8), 6.39(\mathrm{dd}, 1 \mathrm{H}, J=2.0$, 8.4), $6.31(\mathrm{~m}, 2 \mathrm{H}), 6.23(\mathrm{~m}, 2 \mathrm{H}), 5.39(\mathrm{bs}, 1 \mathrm{H}), 4.91(\mathrm{dd}, 1 \mathrm{H}, J=2.0,8.4)$, $4.33(\mathrm{~m}, 2 \mathrm{H}), 4.16(\mathrm{q}, 2 \mathrm{H}, J=7.2), 3.92(\mathrm{~m}, 1 \mathrm{H}), 2.94(\mathrm{~s}, 6 \mathrm{H}), 1.28(\mathrm{t}, 3 \mathrm{H}$, $J=7.2) \mathrm{ppm} ;{ }^{13} \mathrm{C}$ NMR $\left(100 \mathrm{MHz}, \mathrm{CDCl}_{3}\right): \delta 214.0,158.2,155.0,139.0$, $130.1,129.9,128.5,128.1,127.9,126.1106 .3,103.0,100.7,74.3,69.1,68.1,66.6,64.0,40.5,14.2$ ppm; IR (neat): 2912, 1744, 1647, 1612, 1569, 1499, 1449, 1262, $1149 \mathrm{~cm}^{-1} ;[\alpha]_{\mathrm{D}}^{23}=+44^{\circ}(\mathrm{c}=1.0$ $\mathrm{CH}_{2} \mathrm{Cl}_{2}$ ). 


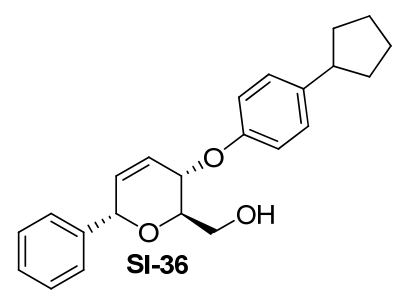

$1068 \mathrm{~cm}^{-1} ;[\alpha]_{\mathrm{D}}^{23}=+92^{\circ}\left(\mathrm{c}=1.0 \mathrm{CH}_{2} \mathrm{Cl}_{2}\right)$.

Aryl ether SI-36: Yield: $22 \mathrm{mg}(0.12 \mathrm{mmol}, 83 \%)$ as a film. ${ }^{1} \mathrm{H}$ NMR $\left(400 \mathrm{MHz}, \mathrm{CDCl}_{3}\right): \delta$ 7.31-7.46 (m, 5H), $7.17(\mathrm{~d}, 2 \mathrm{H}, J=8.0), 7.88(\mathrm{~d}, 2 \mathrm{H}$, $J=8.8), 5.37(\mathrm{~m}, 1 \mathrm{H}), 4.88(\mathrm{~m}, 1 \mathrm{H}), 3.74(\mathrm{~m}, 3 \mathrm{H}), 2.92(\mathrm{~m}, 1 \mathrm{H}), 2.03(\mathrm{~m}$, 2H), $1.67(\mathrm{~m}, 7 \mathrm{H}) \mathrm{ppm} ;{ }^{13} \mathrm{C} \mathrm{NMR}\left(100 \mathrm{MHz}, \mathrm{CDCl}_{3}\right): \delta 155.5,139.4,139.0$, $129.7,128.5,128.2,128.1,128.0,126,7,115.6,74.4,70.8,68.6,62.3,45.1$, 34.7, 25.4 ppm; IR (neat): 3421, 2951, 2854, 1693, 1511, 1449, 1383, 1223,

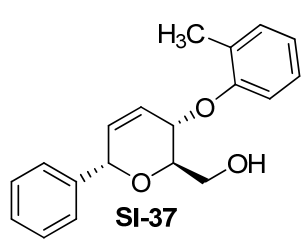

Aryl ether SI-37 Yield: $15 \mathrm{mg}(0.10 \mathrm{mmol}, 68 \%)$ as a film. ${ }^{1} \mathrm{H}$ NMR (400 MHz, $\left.\mathrm{CDCl}_{3}\right): \delta 7.35-7.48(\mathrm{~m}, 5 \mathrm{H}), 7.18(\mathrm{~m}, 2 \mathrm{H}), 6.89(\mathrm{~m}, 2 \mathrm{H}), 6.20(\mathrm{~m}, 2 \mathrm{H}), 5.38(\mathrm{~d}$, $1 \mathrm{H}, J=2.0), 4.91$ (appd, 1H, $J=6.4), 3.80(\mathrm{~m}, 3 \mathrm{H}), 2.19$ (s, 3H) ppm; ${ }^{13} \mathrm{C} \mathrm{NMR}$ $\left(100 \mathrm{MHz}, \mathrm{CDCl}_{3}\right): \delta 155.4,139.1,131.1,129.8,128.5,128.2,128.0,127.6$, $126.8,126.6,121.0,112.5,74.4,71.0,68.5,62.4,16.4$ ppm; IR (neat): 3433, $2920,1604,1491,1456,1386,1235,1192,1083 \mathrm{~cm}^{-1} ;[\alpha]_{\mathrm{D}}^{23}=+45^{\circ}\left(\mathrm{c}=1.0 \mathrm{CH}_{2} \mathrm{Cl}_{2}\right)$.

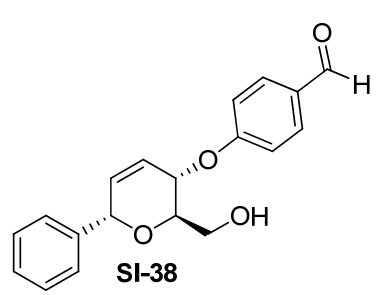

Aryl ether SI-38 Yield: $15 \mathrm{mg}(0.10 \mathrm{mmol}, 68 \%)$ as a film. ${ }^{1} \mathrm{H}$ NMR (400 $\left.\mathrm{MHz}, \mathrm{CDCl}_{3}\right): \delta 9.89(\mathrm{~s}, 1 \mathrm{H}), 7.85(\mathrm{~d}, 2 \mathrm{H}, J=8.4), 7.42(\mathrm{~m}, 5 \mathrm{H}), 7.06(\mathrm{~d}, 2 \mathrm{H}$, $J=8.4), 6.24$ (m, 1H), 6.16 (d, 1H, $J=10.4), 5.39$ (d, 1H, $J=1.6), 5.10$ (dd, 1H, $J=1.2,7.6), 3.76(\mathrm{~m}, 3 \mathrm{H}), 1.95$ (bs, $1 \mathrm{H}) \mathrm{ppm} ;{ }^{13} \mathrm{C} \mathrm{NMR}\left(100 \mathrm{MHz}, \mathrm{CDCl}_{3}\right)$ : $\delta 190.7,162.5,138.6,132.1,130.3,128.6,128.4,128.1,125.4,115.7,74.5$, 70.4, 68.1, 61.8 ppm; IR (neat): 3421, 2916, 2361, 1957, 1682, 1600, 1510, $1386,1239,1165 \mathrm{~cm}^{-1} ;[\alpha]_{\mathrm{D}}^{23}=+98^{\circ}\left(\mathrm{c}=1.0 \mathrm{CH}_{2} \mathrm{Cl}_{2}\right)$.

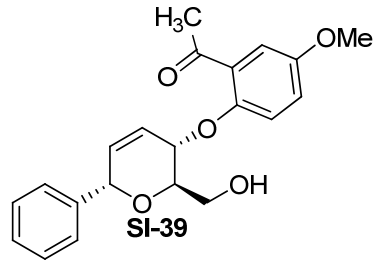

(c $=1.0 \mathrm{CH}_{2} \mathrm{Cl}_{2}$ ).

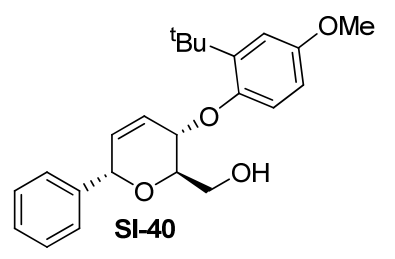

Aryl ether SI-39: Yield: $27 \mathrm{mg}(0.05 \mathrm{mmol}, 38 \%)$ as a film. ${ }^{1} \mathrm{H}$ NMR (400 $\left.\mathrm{MHz} \mathrm{CDCl}_{3}\right): \delta 7.38(\mathrm{~m}, 5 \mathrm{H}), 7.27(\mathrm{~s}, 1 \mathrm{H}), 7.03(\mathrm{bs}, 2 \mathrm{H}), 6.17(\mathrm{~m}, 2 \mathrm{H}), 5.37$ (bs, 1H), 5.02 (d, 1H, J=6.8), $3.80(\mathrm{~m}, 6 \mathrm{H}), 2.56$ (s, 3H) ppm; ${ }^{13} \mathrm{C}$ NMR (100 $\left.\mathrm{MHz}, \mathrm{CDCl}_{3}\right): \delta 199.3,153.9,150.5,138.6,130.7,129.9,128.6,128.4$, $128.0,125.5,120.4,115.7,114.0,74.4,70.8,69.3,62.1,55.8,32.2 \mathrm{ppm}$; IR (neat): $3421,2912,1662,1491,1414,1285,1219,1048 \mathrm{~cm}^{-1} ;[\alpha]_{\mathrm{D}}^{23}=+52^{\circ}$

34.9, 29.8 ppm; IR (neat): 3425, 2955, 2924, 1957, 1728, 1576, 1484, 1456, 1383, 1266, 1208, 1095, $1052 \mathrm{~cm}^{-1} ;[\alpha]_{\mathrm{D}}^{23}=+77^{\mathrm{o}}\left(\mathrm{c}=1.0 \mathrm{CH}_{2} \mathrm{Cl}_{2}\right)$. 


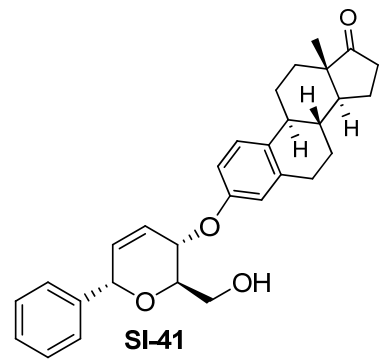

Aryl ether SI-41: Yield: $24 \mathrm{mg}(0.10 \mathrm{mmol}, 68 \%)$ as a film. ${ }^{1} \mathrm{H}$ NMR (400 $\left.\mathrm{MHz}, \mathrm{CDCl}_{3}\right): \delta 7.40(\mathrm{~m}, 5 \mathrm{H}), 4.22(\mathrm{~d}, 1 \mathrm{H}, J=8.0), 4.77(\mathrm{dd}, 1 \mathrm{H}, J=2.8,8.4)$, $6.71(\mathrm{~d}, 1 \mathrm{H}, J=2.8), 6.20$ (dt, $1 \mathrm{H}, J=2.0,10.4), 6.14(\mathrm{dq}, 1 \mathrm{H}, J=1.6,2.8$, 10.4), 5.36 (d, $1 \mathrm{H}, J=2.0), 4.88$ (dt, $1 \mathrm{H}, J=2.0,6.4), 3.73(\mathrm{~m}, 3 \mathrm{H}), 2.89(\mathrm{~m}$, $2 \mathrm{H}), 2.52(\mathrm{~m}, 1 \mathrm{H}), 2.41(\mathrm{~m}, 1 \mathrm{H}), 2.24(\mathrm{~m}, 1 \mathrm{H}), 2.03(\mathrm{~m}, 4 \mathrm{H}), 1.57(\mathrm{~m}, 6 \mathrm{H})$, 0.92 (s, 3H) ppm; ${ }^{13} \mathrm{C}$ NMR (100 MHz, $\left.\mathrm{CDCl}_{3}\right): \delta 155.4,138.9,138.1,132.8$, $129.8,128.5,128.2,128.1,126.7,126.6,115.9,113.3,74.5,70.7,68.4,62.3$, 50.4, 48.0, 44.0, 38.3, 35.9, 31.6, 29.6, 26.5, 21.6, 13.8 ppm; IR (neat): 3724, 3421, 2916, 1732, 1608, $1495,1390,1247,1068 \mathrm{~cm}^{-1} ;[\alpha]_{\mathrm{D}}^{23}=+150^{\circ}\left(\mathrm{c}=1.0 \mathrm{CH}_{2} \mathrm{Cl}_{2}\right)$.

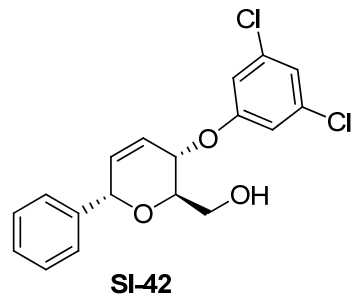

Aryl ether SI-42: Yield: $72 \mathrm{mg}(0.04 \mathrm{mmol}, 30 \%)$ as a film. ${ }^{1} \mathrm{H}$ NMR (400 $\left.\mathrm{MHz}, \mathrm{CDCl}_{3}\right): \delta 7.40(\mathrm{~m}, 5 \mathrm{H}), 6.99(1 \mathrm{H}, \mathrm{t}, J=1.6), 6.86$ (appd, $2 \mathrm{H}, J=1.6$ ), $6.21(\mathrm{ddd}, 1 \mathrm{H}, J=10.4,2.8,1.6), 6.13(\mathrm{dt}, 1 \mathrm{H}, J=1.6,10.4), 5.37(1 \mathrm{H}, \mathrm{q}$, $J=2.4), 4.93(\mathrm{~m}, 1 \mathrm{H}), 3.70(\mathrm{~m}, 3 \mathrm{H}), 1.92(\mathrm{t}, 1 \mathrm{H}, J=7.2) \quad \mathrm{ppm} ;{ }^{13} \mathrm{C} \mathrm{NMR}(100$ $\left.\mathrm{MHz}, \mathrm{CDCl}_{3}\right): \delta 158.5,138.6,135.6,130.9,128.6,128.3,128.1,125.3,121.7$, 114.7, 74.5, 70.4, 68.7, 61.8 ppm; IR (neat): 3425, 3079, 2951, 2920, 1953, $1584,1573,1456,1386,1254,1095 \mathrm{~cm}^{-1} ;[\alpha]_{\mathrm{D}}^{23}=+82^{\circ}\left(\mathrm{c}=1.0 \mathrm{CH}_{2} \mathrm{Cl}_{2}\right)$.

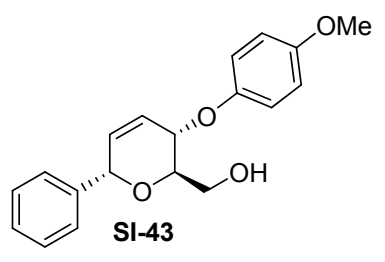

Aryl ether SI-43 (Compound 33): Yield: $34 \mathrm{mg}(0.11 \mathrm{mmol}, 80 \%)$ as a film. ${ }^{1} \mathrm{H}$ NMR $\left(400 \mathrm{MHz}, \mathrm{CDCl}_{3}\right): \delta$ 7.34-7.46 (m, 5H), $6.91(\mathrm{~d}, 1 \mathrm{H}, J=9.2)$, 6.85 (d, 1H, $J=9.2), 6.85$ (d, 1H, $J=9.2), 6.20$ (dt, $1 \mathrm{H}, J=1.6,10.4), 6.15$ (ddd, $1 \mathrm{H}, J=1.2,2.8,10.4$ ), 5.36 (appd, $1 \mathrm{H}, J=2.0$ ), 4.81 (dd, $1 \mathrm{H}, J=1.6$, 8.0), $3.75(\mathrm{~m}, 6 \mathrm{H}), 1.86$ (bs, $1 \mathrm{H}) \mathrm{ppm} ;{ }^{13} \mathrm{C} \mathrm{NMR}\left(100 \mathrm{MHz}, \mathrm{CDCl}_{3}\right): \delta$ 154.3, 151.4, 138.9, 129.7, 128.5, 128.1, 128.1, 126.6, 117.2, 114.8, 74.4, 70.9, 69.5, 62.2, 55.7 ppm; IR (neat): 4352, 2908, 1511, 1449, 1382, 1223, 1037, 816, $746 \mathrm{~cm}^{-1}$; HRMS (Tof) [M+H] ${ }^{+}$: calcd. for $\mathrm{C}_{19} \mathrm{H}_{21} \mathrm{O}_{4} 331.1440$, found 313.1495. $[\alpha]_{\mathrm{D}}^{23}=+95^{\circ}\left(\mathrm{c}=1.0 \mathrm{CH}_{2} \mathrm{Cl}_{2}\right)$.

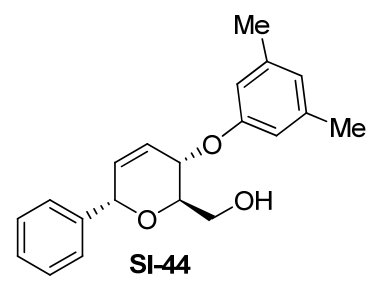

Aryl ether SI-44: Yield: XXmg (0.12 mmol, XX\%) as a film. ${ }^{1} \mathrm{H}$ NMR (400 $\left.\mathrm{MHz}, \mathrm{CDCl}_{3}\right): \delta$ 7.34-7.46 (m, 5H), $6.61(\mathrm{~d}, 3 \mathrm{H}, J=19.6), 6.21(\mathrm{ad}, 2 \mathrm{H}$, $J=12.0$ ), 6.15 (as, $2 \mathrm{H}, J=10.4$ ), 5.37, (d, $1 \mathrm{H}, J=2.0$ ), 4.89 (dt, $1 \mathrm{H}, 1.6,6.4$ ), $3.73(\mathrm{~m}, 3 \mathrm{H}), 2.29$ (bs, 6H) ppm; ${ }^{13} \mathrm{C} \mathrm{NMR}\left(100 \mathrm{MHz}, \mathrm{CDCl}_{3}\right): \delta 157.4$, $139.5,139.0,129.7,128.5,128.2,128.1,126.7,123.1,113.5,74.5,70.8,68.3$, 62.3, 21.4 ppm; IR (neat): 3398, 2920, 2957, 1592, 1460, 1390, 1289, 1157 $\mathrm{cm}^{-1} ;[\alpha]_{\mathrm{D}}^{23}=+101^{\circ}\left(\mathrm{c}=1.0 \mathrm{CH}_{2} \mathrm{Cl}_{2}\right)$. 
Table SI-6. C-glycosides prepared via the Pd-mediated aryl-etherification.
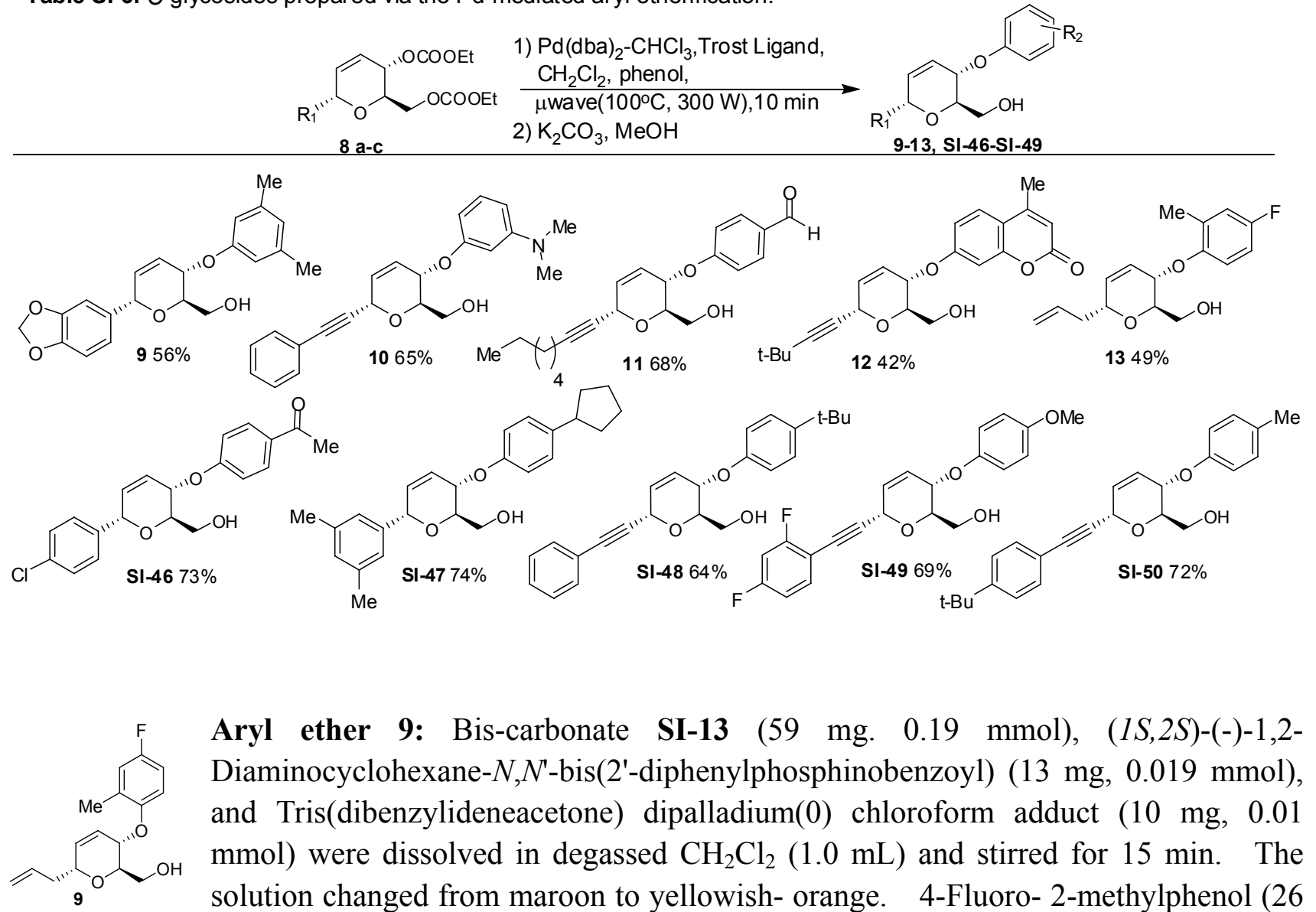

Aryl ether 9: Bis-carbonate SI-13 (59 mg. 0.19 mmol), (1S,2S)-(-)-1,2Diaminocyclohexane- $N, N$ '-bis(2'-diphenylphosphinobenzoyl) (13 $\mathrm{mg}, 0.019 \mathrm{mmol})$, and Tris(dibenzylideneacetone) dipalladium $(0)$ chloroform adduct $(10 \mathrm{mg}, 0.01$ mmol) were dissolved in degassed $\mathrm{CH}_{2} \mathrm{Cl}_{2}(1.0 \mathrm{~mL})$ and stirred for $15 \mathrm{~min}$. The solution changed from maroon to yellowish- orange. 4-Fluoro- 2-methylphenol (26 $\mathrm{mg}, 0.21 \mathrm{mmol})$ was added and the reaction was irradiated for $15 \mathrm{~min}(150-300 \mathrm{~W}$, Powermax enabled) at $100^{\circ} \mathrm{C}$. The crude reaction mixture was transferred to a $20 \mathrm{~mL}$ scintillation vial and concentrated in vacuo. The reaction was resuspended in $\mathrm{MeOH}(3.0 \mathrm{~mL})$ and $\mathrm{K}_{2} \mathrm{CO}_{3}(300$ $\mathrm{mg}, 2.0 \mathrm{mmol}$ ) was added. After stirring for $5 \mathrm{~h}$, the reaction was concentrated and purified by $\mathrm{SiO}_{2}$ chromatography (0-40\% ethyl acetate / hexanes) to provide 13 (26 mg, 49\%) as a film. ${ }^{1} \mathrm{H}$ NMR (400 $\left.\mathrm{MHz}, \mathrm{CDCl}_{3}\right): \delta 6.86(\mathrm{~m}, 3 \mathrm{H}), 5.91(\mathrm{~m}, 3 \mathrm{H}), 5.14(\mathrm{~m}, 2 \mathrm{H}), 4.65(\mathrm{dd}, 1 \mathrm{H}, J=1.6,7.0), 4.33(\mathrm{~m}, 1 \mathrm{H})$, $3.84(\mathrm{~m}, 2 \mathrm{H}), 3.72(\mathrm{~m}, 1 \mathrm{H}), 2.53(\mathrm{~m}, 1 \mathrm{H}), 2.35(\mathrm{~m}, 1 \mathrm{H}), 2.19(\mathrm{~s}, 3 \mathrm{H}), 2.07(\mathrm{~m}, 1 \mathrm{H}) \mathrm{ppm} ;{ }^{13} \mathrm{C}$ NMR $\left(100 \mathrm{MHz}, \mathrm{CDCl}_{3}\right): \delta 158.3,155.9,151.3,134.5,131.4,129.5,125.2,117.6,113.5,112.5,72.6,71.2$, 69., 62.4, 37.6, 16.5 ppm; IR (neat): 3440, 2917, 1499, 1262, 1204, 1087, 1045, $796 \mathrm{~cm}^{-1} ;[\alpha]_{\mathrm{D}}{ }^{23}=$ $+93^{\circ}\left(\mathrm{c}=1.0 \mathrm{CH}_{2} \mathrm{Cl}_{2}\right)$.

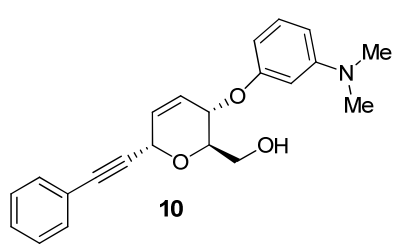

Aryl ether 10: Bis-carbonate SI-26 (100 mg. $0.27 \mathrm{mmol}),(1 S, 2 S)-(-)-1,2-$ Diaminocyclohexane- N,N'-bis(2'-diphenylphosphinobenzoyl) (9 mg, 0.01 mmol), and Tris(dibenzylideneacetone) dipalladium $(0)$ chloroform adduct ( $7 \mathrm{mg}, 0.01 \mathrm{mmol}$ ) were dissolved in degassed $\mathrm{CH}_{2} \mathrm{Cl}_{2}(1.0 \mathrm{~mL})$ and stirred for 15 min. The color of the solution changed from maroon to yellowish-orange. 3-dimethylamino phenol $(40 \mathrm{mg}, 0.3 \mathrm{mmol})$ was added and the reaction was irradiated for $15 \mathrm{~min}\left(150-300 \mathrm{~W}\right.$, Powermax enabled) at $100^{\circ} \mathrm{C}$. The crude reaction mixture was 
transferred to a $20 \mathrm{~mL}$ scintillation vial and concentrated in vacuo. The reaction was resuspended in $\mathrm{MeOH}(2.0 \mathrm{~mL})$ and MP-carbonate resin $(20 \mathrm{mg}, 0.1 \mathrm{mmol})$ was added. After stirring for $6 \mathrm{~h}$, the reaction was concentrated and purified by $\mathrm{SiO}_{2}$ chromatography $(0-70 \%$ ethyl acetate / hexanes) to provide $10(59 \mathrm{mg}, 65 \%)$ as a film. ${ }^{1} \mathrm{H}$ NMR (400 $\left.\mathrm{MHz}, \mathrm{CDCl}_{3}\right): \delta 7.47(\mathrm{~m}, 2 \mathrm{H}), 7.33(\mathrm{~m}, 3 \mathrm{H})$, 7.15 (t, $1 \mathrm{H}, J=6.8), 6.38(\mathrm{~m}, 3 \mathrm{H}), 6.06(\mathrm{~d}, 1 \mathrm{H}, J=2.0,10.0), 5.95(\mathrm{ddd}, 1 \mathrm{H}, J=1.6,3.2,10.4), 5.24$ (m, $1 \mathrm{H}), 4.90$ (dd, 1H, $J=2.0,8.8), 4.15$ (m, 1H), 3.99 (m, 1H, $J=2.8,12.0), 3.82$ (dd, $1 \mathrm{H}, J=5.2,12.0$ ), 2.91 (bs, 6H) ppm; ${ }^{13} \mathrm{C}$ NMR $\left(100 \mathrm{MHz}, \mathrm{CDCl}_{3}\right): \delta 158.4,152.1,131.9,129.9,128.6,128.3,128.2$, 126.4, 122.3, 106.3, 103.0, 100.7, 86.4, 85.2, 72.8, 68.0, 64.6, 62.3, 40.5 ppm; IR (neat): 3409, 2924, $2361,1607,1581,1503,1449,1390,1235,1153,1079,757 \mathrm{~cm}^{-1} ;[\alpha]_{\mathrm{D}}^{23}=+12^{\circ}\left(\mathrm{c}=1.0 \mathrm{CH}_{2} \mathrm{Cl}_{2}\right)$.

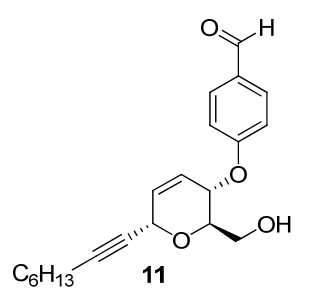

Aryl ether 11: Bis-carbonate SI-28 (0.081g. $0.21 \mathrm{mmol})$, (1S,2S)- (-)- 1,2Diaminocyclohexane- N,N'-bis(2'-diphenylphosphinobenzoyl) (15 mg, 0.021 $\mathrm{mmol}$ ), and Tris(dibenzylideneacetone) dipalladium(0) chloroform adduct (10 $\mathrm{mg}$, $0.01 \mathrm{mmol})$ were dissolved in degassed $\mathrm{CH}_{2} \mathrm{Cl}_{2}(1.0 \mathrm{~mL})$ and stirred for $15 \mathrm{~min}$. The color of the solution changed from maroon to yellowish-orange. 4-Hydroxybenzaldehyde $(28 \mathrm{mg}, 0.20 \mathrm{mmol})$ was added and the reaction was irradiated for $15 \mathrm{~min}\left(150-300 \mathrm{~W}\right.$, Powermax enabled) at $100^{\circ} \mathrm{C}$. The crude reaction mixture was transferred to a $20 \mathrm{~mL}$ scintillation vial and concentrated in vacuo. The reaction was resuspended in $\mathrm{MeOH}(3.0 \mathrm{~mL})$ and $\mathrm{K}_{2} \mathrm{CO}_{3}(71 \mathrm{mg}(0.21 \mathrm{mmol})$ was added. After stirring for $15 \mathrm{~h}$, the reaction was concentrated and purified by $\mathrm{SiO}_{2}$ chromatography (0-70\% ethyl acetate / hexanes) to provide 11 (49 mg, 68\%) as a film. ${ }^{1} \mathrm{H}$ NMR (400 MHz, $\left.\mathrm{CDCl}_{3}\right): \delta 9.88(\mathrm{~s}, 1 \mathrm{H}), 7.83(\mathrm{~d}, 2 \mathrm{H}, J=9.2), 7.03(\mathrm{~d}, 2 \mathrm{H}$, $J=8.8), 5.91(\mathrm{~m}, 2 \mathrm{H}), 5.02(\mathrm{~m}, 2 \mathrm{H}), 4.08(\mathrm{~m}, 1 \mathrm{H}), 3.93(\mathrm{dd}, 1 \mathrm{H}, J=2.0,12.0), 3.75$ (dd, $1 \mathrm{H}, J=4.4$, 12.0), $2.24(\mathrm{~m}, 2 \mathrm{H}), 1.52(\mathrm{~m}, 2 \mathrm{H}), 1.33(\mathrm{~m}, 6 \mathrm{H}), 0.89(\mathrm{t}, 3 \mathrm{H}, J=6.8) \mathrm{ppm} ;{ }^{13} \mathrm{C}$ NMR $(100 \mathrm{MHz}$, $\left.\mathrm{CDCl}_{3}\right): \delta 190.6,162.4,132.1,130.3,130.2,124.1,115.6,87.9,75.8,71.8,67.9,64.4,61.8,31.2$, 28.5, 28.4, 22.5, 18.7, 14.0 ppm; IR (neat): 3440, 2932, 2858, 1697, 1603, 1577, 1511, 1239, 1161, $1080 \mathrm{~cm}^{-1} ;[\alpha]_{\mathrm{D}}^{23}=+85^{\circ}\left(\mathrm{c}=1.0 \mathrm{CH}_{2} \mathrm{Cl}_{2}\right)$.

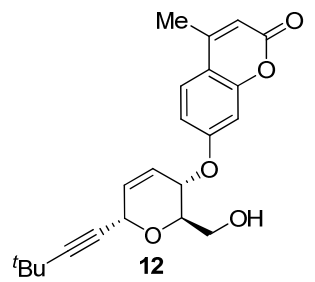

Aryl ether 12: Bis-carbonate SI-29 (70 mg. $0.20 \mathrm{mmol}),(1 S, 2 S)-(-)-1,2-$ Diaminocyclohexane- N,N'-bis(2'-diphenylphosphinobenzoyl) (14 mg, 0.021 $\mathrm{mmol}$ ), and Tris(dibenzylideneacetone)dipalladium(0) chloroform adduct (10 $\mathrm{mg}$, $0.01 \mathrm{mmol})$ were dissolved in degassed $\mathrm{CH}_{2} \mathrm{Cl}_{2}(1.0 \mathrm{~mL})$ and stirred for $15 \mathrm{~min}$.

The solution changed from maroon to yellowish-orange. 7- Hydroxy -4-methylcoumarin (39 $\mathrm{mg}, 0.22 \mathrm{mmol}$ ) was added and the reaction was irradiated for $15 \mathrm{~min}\left(150-300 \mathrm{~W}\right.$, Powermax enabled) at $100^{\circ} \mathrm{C}$. The crude reaction mixture was transferred to a $20 \mathrm{~mL}$ scintillation vial and concentrated in vacuo. The reaction was resuspended in $\mathrm{MeOH}(3.0 \mathrm{~mL})$ and $\mathrm{K}_{2} \mathrm{CO}_{3}(370 \mathrm{mg}, 2.7 \mathrm{mmol})$ was added. After stirring for $15 \mathrm{~h}$, the reaction was concentrated and purified by $\mathrm{SiO}_{2}$ chromatography (0-60\% ethyl acetate / hexanes) to provide 12 (31 mg, 42\%) as a film. ${ }^{1} \mathrm{H}$ NMR (400 MHz, $\left.\mathrm{CDCl}_{3}\right): \delta 7.51(\mathrm{~d}, 1 \mathrm{H}, J=8.4), 6.88(\mathrm{~m}, 2 \mathrm{H}), 6.15$ (bs, 1H), $5.90(\mathrm{~s}, 2 \mathrm{H}), 5.02(\mathrm{~s}, 1 \mathrm{H}), 4.94(\mathrm{dd}, 1 \mathrm{H}, 1.6,8.4), 4.05(\mathrm{~m}, 1 \mathrm{H}), 3.94(\mathrm{~m}, 1 \mathrm{H}), 3.76(\mathrm{~m}, 1 \mathrm{H})$, 2.39 (s, 3H), 1.23 (s, 9H) ppm; ${ }^{13} \mathrm{C}$ NMR (100 MHz, $\left.\mathrm{CDCl}_{3}\right): \delta 161.1,160.3,155.2,152.4,130.4$, $125.8,123.9,114.1,112.9,112.3,102.8,95.9,74.1,71.7,68.3,64.4,61.9,30.8,27.5,18.7$ ppm; IR 
(neat): $3441,2967,2920,2233,1724,1611,1386,1262,1153,1072 \mathrm{~cm}^{-1}$; HRMS (Tof) $[\mathrm{M}+\mathrm{Na}]^{+}$: calcd. for $\mathrm{C}_{22} \mathrm{H}_{24} \mathrm{O}_{5} \mathrm{Na} 391.1521$, found 391.1508. $[\alpha]_{\mathrm{D}}{ }^{23}=+82^{\circ}\left(\mathrm{c}=1.0 \mathrm{CH}_{2} \mathrm{Cl}_{2}\right)$.

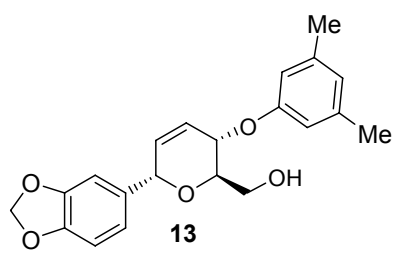

Aryl ether 13: Dicarbonate SI-21 (125 mg $0.32 \mathrm{mmol}),(1 S, 2 S)-(-)-1,2-$ Diaminocyclohexane- N,N'-bis(2'-diphenylphosphinobenzoyl) (11 mg, $0.02 \mathrm{mmol})$, and Tris(dibenzylideneacetone) dipalladium $(0)$ chloroform adduct $\left(8 \mathrm{mg}, 0.02 \mathrm{mmol}\right.$ ) were dissolved in degassed $\mathrm{CH}_{2} \mathrm{Cl}_{2}(2.0 \mathrm{~mL})$ and stirred for $15 \mathrm{~min}$. The color of the solution changed from maroon to yellowish-orange. 3,5-Dimethyl phenol $(41 \mathrm{mg}, 0.3 \mathrm{mmol})$ was added and the reaction was irradiated for $15 \min \left(150-300 \mathrm{~W}\right.$, Powermax enabled) at $100^{\circ} \mathrm{C}$. The crude reaction mixture was concentrated onto $\mathrm{SiO}_{2}$ and chromatographed (0-40\% ethyl acetate/hexanes) to provide $121 \mathrm{mg}$ of ethyl carbonate-protected 9. The intermediate was resuspended in $\mathrm{MeOH}(1.0 \mathrm{~mL})$ and $\mathrm{MP}-\mathrm{CO}_{3}$ $(11 \mathrm{mg}, 2.98 \mathrm{mmol} / \mathrm{g}$ ) was added. After stirring for $3 \mathrm{~h}$, the reaction was concentrated and purified by $\mathrm{SiO}_{2}$ chromatography ( $0-70 \%$ ethyl acetate / hexanes) to provide $9(33 \mathrm{mg}, 56 \%)$ as a film. ${ }^{1} \mathrm{H}$ NMR (400 MHz, $\left.\mathrm{CDCl}_{3}\right): \delta 6.95(\mathrm{~m}, 1 \mathrm{H}), 6.91(\mathrm{~d}, 1 \mathrm{H}, \mathrm{J}=7.6), 6.80(\mathrm{~d}, 1 \mathrm{H}, \mathrm{J}=8.0), 6.63(\mathrm{~s}, 1 \mathrm{H})$, $6.58(\mathrm{~s}, 2 \mathrm{H}), 6.2(\mathrm{~m} \mathrm{1H}), 6.09(\mathrm{~m}, 1 \mathrm{H}), 5.98(\mathrm{~s}, 2 \mathrm{H}), 5.25(\mathrm{~m}, 1 \mathrm{H}), 4.86(\mathrm{dd}, 1 \mathrm{H}, \mathrm{J}=1.6,8.0), 3.71(\mathrm{~m}$, $3 \mathrm{H}), 2.29$ (s, 6H) ppm; ${ }^{13} \mathrm{C}$ NMR $\left(100 \mathrm{MHz}, \mathrm{CDCl}_{3}\right): \delta 157.3,147.8,147.6,139.4,132.8,129.7$, $126.8,123.1,121.9,113.5,108.9,108.1,101.1,74.2,70.5,68.3,62.3,21.4$ ppm; IR (neat): 3433, $2909,1713,1596,1487,1441,1285,1223,1161,1072,928 \mathrm{~cm}^{-1} ;[\alpha]_{\mathrm{D}}{ }^{23}=+68^{\circ}\left(\mathrm{c}=1.0 \mathrm{CH}_{2} \mathrm{Cl}_{2}\right)$.

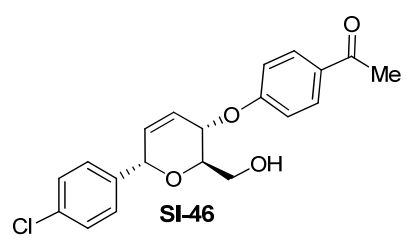

Aryl ether SI-46: Bis-carbonate SI-22 (111 mg. $0.29 \mathrm{mmol}),(1 S, 2 S)-(-)-$ 1,2- Diaminocyclohexane- N,N'-bis(2'-diphenylphosphinobenzoyl) $(10 \mathrm{mg}$, $0.014 \mathrm{mmol}$ ), and Tris(dibenzylideneacetone) dipalladium(0) chloroform adduct $(8 \mathrm{mg}, 0.007 \mathrm{mmol})$ were dissolved in degassed $\mathrm{CH}_{2} \mathrm{Cl}_{2}(1.5 \mathrm{~mL})$ and stirred for $15 \mathrm{~min}$. The solution changed from maroon to yellowishorange. 4'-hydroxyacetophenone ( $41 \mathrm{mg}, 0.30 \mathrm{mmol})$ was added and the reaction was irradiated for 15 min (150-300 W, Powermax enabled) at $100^{\circ} \mathrm{C}$. The reaction mixture concentrated onto $\mathrm{SiO}_{2}$ and chromatographed over $\mathrm{SiO}_{2}(0-50 \%$ ethyl acetate / pet. ether) to provide the crude carbonate (104 mg, approx 84\%) as a viscous oil. The mixture was resuspended in $\mathrm{MeOH}(3.0 \mathrm{~mL}$ ) and $\mathrm{K}_{2} \mathrm{CO}_{3}(8 \mathrm{mg}, 0.05 \mathrm{mmol})$ was added. After stirring for $6 \mathrm{~h}$, the reaction was concentrated and purified by $\mathrm{SiO}_{2}$ chromatography (0-40\% ethyl acetate / hexanes) to provide $\mathbf{S I - 4 6 ~ ( 5 9 ~} \mathrm{mg}, 73 \%$ ) as a film. ${ }^{1} \mathrm{H}$ NMR (400 MHz, $\left.\mathrm{CDCl}_{3}\right): \delta 7.94(\mathrm{~d}, 2 \mathrm{H}, J=8.8), 7.37$ (s, 4H), 6.98 (d, 2H, $\left.J=9.2\right), 6.17(2$, $2 \mathrm{H}), 5.34(\mathrm{~m}, 1 \mathrm{H}), 5.05(\mathrm{dd}, 1 \mathrm{H}, J=2.0,7.6), 3.73(\mathrm{~m}, 3 \mathrm{H}), 2.56(\mathrm{~s}, 3 \mathrm{H}), 1.99(\mathrm{t},-\mathrm{OH}) \mathrm{ppm} ;{ }^{13} \mathrm{C}$ NMR $\left(100 \mathrm{MHz}, \mathrm{CDCl}_{3}\right): \delta 196.7,161.3,137.2,134.3,130.7,130.2,129.4,128.8,126.0,115.1,73.7$, 70.6, 68.0, 61.8, $26.3 \mathrm{ppm}$; IR (neat): 3425, 1674, 1592, 1506, 1250, 1173, $1087 \mathrm{~cm}^{-1} ; \quad$ HRMS (Tof) $[\mathrm{M}+\mathrm{Na}]^{+}$: calcd. for $\mathrm{C}_{20} \mathrm{H}_{19} \mathrm{O}_{4} \mathrm{NaCl} 381.0870$, found 381.0887. $[\alpha]_{\mathrm{D}}^{23}=+120^{\circ}\left(\mathrm{c}=1.0 \mathrm{CH}_{2} \mathrm{Cl}_{2}\right)$.

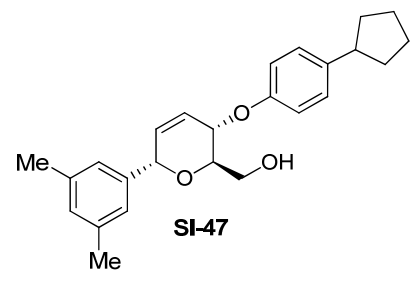

Aryl ether SI-47: Bis-carbonate SI-25 (108 mg. $0.29 \mathrm{mmol})$, (IS,2S)- (-)1,2- Diaminocyclohexane- N,N'-bis(2'-diphenylphosphinobenzoyl) (10 mg, $0.014 \mathrm{mmol}$ ), and Tris(dibenzylideneacetone) dipalladium(0) chloroform adduct $(8 \mathrm{mg}, 0.007 \mathrm{mmol})$ were dissolved in degassed $\mathrm{CH}_{2} \mathrm{Cl}_{2}(1.0 \mathrm{~mL})$ 
and stirred for $15 \mathrm{~min}$. The solution changed from maroon to yellowish- orange. 4-cyclopentylphenol (47 mg, $0.29 \mathrm{mmol}$ ) was added and the reaction was irradiated for $15 \mathrm{~min}$ (150-300 W, Powermax enabled) at $100^{\circ} \mathrm{C}$. The reaction mixture concentrated onto $\mathrm{SiO}_{2}$ and chromatographed over $\mathrm{SiO}_{2}(0-50 \%$ ethyl acetate / pet. ether) to provide the crude carbonate $(120 \mathrm{mg}$, approx $80 \%)$ as a viscous oil. The mixture was resuspended in $\mathrm{MeOH}(2.0 \mathrm{~mL})$ and $\mathrm{K}_{2} \mathrm{CO}_{3}(6.3 \mathrm{mg}$, $0.05 \mathrm{mmol}$ ) was added. After stirring for $5 \mathrm{~h}$, the reaction was concentrated and purified by $\mathrm{SiO}_{2}$ chromatography (0-40\% ethyl acetate / hexanes) to provide SI-47 (64 mg, 74\%) as a film. ${ }^{1} \mathrm{H}$ NMR $\left(400 \mathrm{MHz}, \mathrm{CDCl}_{3}\right): \delta 7.18(\mathrm{~d}, 2 \mathrm{H}, J=2.0), 7.05(\mathrm{~s}, 2 \mathrm{H}), 6.98(\mathrm{~s}, 1 \mathrm{H}), 6.91(\mathrm{~d}, 2 \mathrm{H}, J=6.4), 6.19$ (dt, $1 \mathrm{H}, J=2.0,12), 6.11$ (dq, $1 \mathrm{H}, J=1.6,2.8,10.4), 5.29$ (m, 1H), 4.88 (dq, $1 \mathrm{H}, J=1.6,3.6,5.6), 3.77$ (m, $3 \mathrm{H}), 2.95(\mathrm{~m}, 1 \mathrm{H}), 2.35(\mathrm{~m}, 2 \mathrm{H}), 2.04(\mathrm{~m}, 2 \mathrm{H}), 1.78(\mathrm{~m}, 2 \mathrm{H}), 1.69(\mathrm{~m}, 2 \mathrm{H}), 1.58(\mathrm{~m}, 2 \mathrm{H}) \mathrm{ppm} ;{ }^{13} \mathrm{C}$ NMR (100 MHz, $\left.\mathrm{CDCl}_{3}\right): \delta 155.5,139.4,138.8,138.0,129.9,129.8,128.1,126.4,126.0,115.6,74.6$, 70.8, 68.7, 62.2, 45.1, 34.7, 25.4, 21.3 ppm; IR (neat): 3452, 2931, 2874, 1608, 1518, 1239, 1177 , $1049,831 \mathrm{~cm}^{-1} ;[\alpha]_{\mathrm{D}}^{23}=+78^{\mathrm{o}}\left(\mathrm{c}=1.0 \mathrm{CH}_{2} \mathrm{Cl}_{2}\right)$.

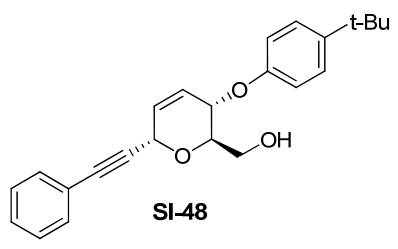

Aryl ether SI-48: Bis-carbonate SI-26 (200 mg. $0.53 \mathrm{mmol}),(1 S, 2 S)-(-)-$ 1,2- Diaminocyclohexane- N,N'-bis(2'-diphenylphosphinobenzoyl) (18 mg, $0.027 \mathrm{mmol})$, and Tris(dibenzylideneacetone) dipalladium $(0)$ chloroform adduct $14 \mathrm{mg}, 0.013 \mathrm{mmol})$ were dissolved in degassed $\mathrm{CH}_{2} \mathrm{Cl}_{2}(1.0 \mathrm{~mL})$ and stirred for $15 \mathrm{~min}$. The solution changed from maroon to yellowishorange. $\quad$-tert-Butyl-phenol $(84 \mathrm{mg}, 0.56 \mathrm{mmol})$ was added and the reaction was irradiated for 15 $\min \left(150-300 \mathrm{~W}\right.$, Powermax enabled) at $100^{\circ} \mathrm{C}$. The reaction mixture concentrated onto $\mathrm{SiO}_{2}$ and chromatographed over $\mathrm{SiO}_{2}(0-50 \%$ ethyl acetate / pet. ether) to provide the crude carbonate $(221 \mathrm{mg}$, approx 95\%) as viscous oil. The mixture was resuspended in $\mathrm{MeOH}(3.0 \mathrm{~mL})$ and $\mathrm{K}_{2} \mathrm{CO}_{3}(34 \mathrm{mg}$, $0.1 \mathrm{mmol}$ ) was added. After stirring for $15 \mathrm{~h}$, the reaction was concentrated and purified by $\mathrm{SiO}_{2}$ chromatography (0-40\% ethyl acetate / hexanes) to provide SI-48 (117 mg, 64\%) as a viscous oil. ${ }^{1} \mathrm{H}$ NMR (400 MHz, $\left.\mathrm{CDCl}_{3}\right): \delta 7.48(\mathrm{~m}, 2 \mathrm{H}), 7.33(\mathrm{~m}, 5 \mathrm{H}), 6.90(\mathrm{~d}, 2 \mathrm{H}, J=8.8), 6.04(\mathrm{~d}, 1 \mathrm{H}, J=10.4)$, 5.95 (dq, 1H, $J=2.0,3.2,10.4), 5.24$ (m, 1H), 4.89 (dd, 1H, $J=1.6,8.8), 4.15$ (m, 1H), 3.98 (dd, 1H, $J=2.8,9.2), 3.81(\mathrm{dd}, 1 \mathrm{H}, J=4.8,11.6), 1.31(\mathrm{~s}, 9 \mathrm{H}) \mathrm{ppm} ;{ }^{13} \mathrm{C} \mathrm{NMR}\left(100 \mathrm{MHz}, \mathrm{CDCl}_{3}\right): \delta 155.0$, $144.3,131.9,128.7,128.3,126.5,126.4,121.1,115.2,86.5,85.3,72.7,68.2,64.6,62.3,34.1,31.5$ ppm; IR (neat): 2955, 1654, 1608, 1507, 1227, $1075 \mathrm{~cm}^{-1}$; HRMS (Tof) $[\mathrm{M}+\mathrm{Na}]^{+}$: calcd. for $\mathrm{C}_{24} \mathrm{H}_{26} \mathrm{O}_{3} \mathrm{Na} 385.1780$, found 385.1769. $[\alpha]_{\mathrm{D}}{ }^{23}=+19^{\circ}\left(\mathrm{c}=1.0 \mathrm{CH}_{2} \mathrm{Cl}_{2}\right)$.

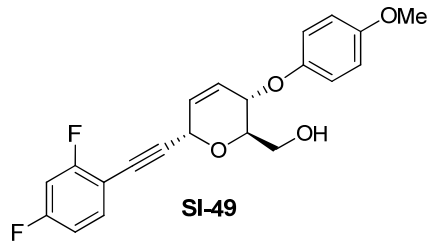

Aryl ether SI-49: Bis-carbonate SI-13 (138 mg. $0.34 \mathrm{mmol}),(1 S, 2 S)-(-)-$ 1,2- Diaminocyclohexane- N,N'-bis(2'-diphenylphosphinobenzoyl) (12 $\mathrm{mg}, \quad 0.017 \mathrm{mmol})$, and Tris(dibenzylideneacetone) dipalladium(0) chloroform adduct ( $9 \mathrm{mg}, 0.008 \mathrm{mmol}$ ) were dissolved in degassed $\mathrm{CH}_{2} \mathrm{Cl}_{2}(1.5 \mathrm{~mL})$ and stirred for $15 \mathrm{~min}$. The solution changed from maroon to yellowish- orange. $p$-Methoxyphenol $(44 \mathrm{mg}, 0.35 \mathrm{mmol}$ ) was added and the reaction was irradiated for $15 \mathrm{~min}\left(150-300 \mathrm{~W}\right.$, Powermax enabled) at $100^{\circ} \mathrm{C}$. The reaction mixture concentrated onto $\mathrm{SiO}_{2}$ and chromatographed over $\mathrm{SiO}_{2}(0-50 \%$ ethyl acetate / pet. ether) to provide the crude carbonate (115 mg, approx 77\%) as a viscous oil. The mixture was resuspended in $\mathrm{MeOH}$ 
$(2.0 \mathrm{~mL})$ and $\mathrm{K}_{2} \mathrm{CO}_{3}(7.2 \mathrm{mg}, 0.05 \mathrm{mmol})$ was added. After stirring for $4 \mathrm{~h}$, the reaction was concentrated and purified by $\mathrm{SiO}_{2}$ chromatography $(0-40 \%$ ethyl acetate / hexanes) to provide SI-49 (67 mg, 69\%) as a film. ${ }^{1} \mathrm{H}$ NMR $\left(400 \mathrm{MHz}, \mathrm{CDCl}_{3}\right): \delta 7.44(\mathrm{~m}, 1 \mathrm{H}), 6.87(\mathrm{~m}, 6 \mathrm{H}), 6.03(\mathrm{dt}, 1 \mathrm{H}$, $J=1.6,9.6), 5.94(\mathrm{dq}, 1 \mathrm{H}, J=1.2,2.8,10.0), 5.24(\mathrm{~m}, 1 \mathrm{H}), 4.79(\mathrm{dq}, 1 \mathrm{H}, J=2.0,3.6,8.8), \quad 4.10(\mathrm{~m}$, $1 \mathrm{H}), 3.98(\mathrm{dd}, 1 \mathrm{H}, J=2.4,12.0), 3.82(\mathrm{dd}, 1 \mathrm{H}, J=4.4,11.6), 3.77$ (s, 3H) ppm; ${ }^{13} \mathrm{C}$ NMR $(100 \mathrm{MHz}$, $\mathrm{CDCl}_{3}$ ): $\delta 163.4(\mathrm{dd}, 1 \mathrm{C}, J=46.8,143.6), 161.8(\mathrm{dd}, 1 \mathrm{C}, J=46.8,134.8), 154.5,151.2,134.6(\mathrm{dd}, 1 \mathrm{C}$, 8.8, 38.4), 127.7, 126.5, 117.2, 114.8, 111.5 (dd, $1 \mathrm{C}, J=17.6,88), 107.1,104.3$ (t, $1 \mathrm{C}, J=96.8), 90.2$, 78.9, 72.9, 69.2, 64.5, 62.2, 55.7 ppm; IR (neat): 3465, 2908, 1619, 1499, 1437, 1223, 1145, 1079, 1036, $827 \mathrm{~cm}^{-1}$; HRMS (Tof) $[\mathrm{M}+\mathrm{H}]^{+}$: calcd. for $\mathrm{C}_{21} \mathrm{H}_{18} \mathrm{O}_{4} \mathrm{~F} 373.1251$, found 373.1253. $[\alpha]_{\mathrm{D}}{ }^{23}=$ $+15^{\circ}\left(\mathrm{c}=1.0 \mathrm{CH}_{2} \mathrm{Cl}_{2}\right)$.

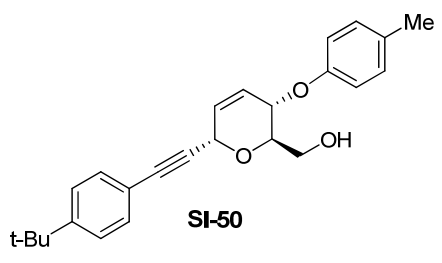

Aryl ether SI-50: Bis-carbonate SI-32 (148 mg. $0.34 \mathrm{mmol}),(1 S, 2 S)-$ (-)- 1,2- Diaminocyclohexane- N,N'-bis(2'-diphenylphosphinobenzoyl) (10 $\mathrm{mg}, 0.014 \mathrm{mmol})$, and Tris(dibenzylideneacetone) dipalladium(0) chloroform adduct $(8 \mathrm{mg}, 0.007 \mathrm{mmol})$ were dissolved in degassed $\mathrm{CH}_{2} \mathrm{Cl}_{2}(1.5 \mathrm{~mL})$ and stirred for $15 \mathrm{~min}$. The solution changed from maroon to yellowish- orange. 4-Methylphenol $(38 \mathrm{mg}, 0.29 \mathrm{mmol}$ ) was added and the reaction was irradiated for $15 \mathrm{~min}\left(150-300 \mathrm{~W}\right.$, Powermax enabled) at $100^{\circ} \mathrm{C}$. The reaction mixture concentrated onto $\mathrm{SiO}_{2}$ and chromatographed over $\mathrm{SiO}_{2}(0-50 \%$ ethyl acetate / pet. ether) to provide the crude carbonate (124 mg, approx 81\%) as a viscous oil. The mixture was resuspended in $\mathrm{MeOH}(2.0 \mathrm{~mL})$ and $\mathrm{K}_{2} \mathrm{CO}_{3}(4 \mathrm{mg}, 0.03 \mathrm{mmol})$ was added. After stirring for $3 \mathrm{~h}$, the reaction was concentrated and purified by $\mathrm{SiO}_{2}$ chromatography ( $0-40 \%$ ethyl acetate / hexanes) to provide $\mathbf{S I - 5 0}(75 \mathrm{mg}, 72 \%)$ as a film. ${ }^{1} \mathrm{H}$ NMR (400 MHz, $\left.\mathrm{CDCl}_{3}\right): \delta 7.41(\mathrm{~d}, 2 \mathrm{H}, J=6.8), 7.34$ (d, $\left.2 \mathrm{H}, J=8.8\right), 7.10(\mathrm{~d}, 1 \mathrm{H}, J=8.0)$, $6.86(\mathrm{~m}, 2 \mathrm{H}), 6.02(\mathrm{dt}, 1 \mathrm{H}, J=1.6,10.4), 5.95(\mathrm{dq}, 1 \mathrm{H}, J=1.6,3.2,10), 5.23(\mathrm{~m}, 1 \mathrm{H}), 4.86(\mathrm{dq}, 1 \mathrm{H}$, $J=1.6,3.2,8.8), 4.15$ (m, $1 \mathrm{H}), 3.97$ (dd, $1 \mathrm{H}, J=2.8,11.6$ ), 3.80 (dd, $1 \mathrm{H}, J=4.8,12.4), 2.30$ (s, $3 \mathrm{H}$ ), 1.32 (s, 9H) ppm; ${ }^{13} \mathrm{C}$ NMR $\left(100 \mathrm{MHz}, \mathrm{CDCl}_{3}\right): \delta 155.1,152.0,131.6,130.8,130.1,128.4,126.0$, $125.3,119.2,115.7,86.6,84.4,72.6,68.4,64.7,62.2,34.8,311,20.5$ ppm; IR (neat): 3441, 3037, $2951,2870,1708,1612,1507,1386,1235,1173,1084,1025,835 \mathrm{~cm}^{-1} ;[\alpha]_{\mathrm{D}}^{23}=+2^{\circ}(\mathrm{c}=1.0$ $\mathrm{CH}_{2} \mathrm{Cl}_{2}$ ).

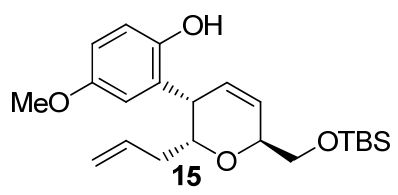

Phenol 15: Aryl ether $14^{2}(110 \mathrm{mg}, 0.28 \mathrm{mmol})$ was dissolved in chlorobenzene $(0.60 \mathrm{~mL})$ in a $10 \mathrm{~mL}$ microwave tube. $\mathrm{Eu}(\mathrm{fod})_{3}(30 \mathrm{mg}$, $0.03 \mathrm{mmol}$ ) was added and the reaction was heated with microwave irradiation $\left(300 \mathrm{~W}, 200^{\circ} \mathrm{C}\right)$ for $30 \mathrm{~min}$. Concentration in vacuo, followed by purification over $\mathrm{SiO}_{2}\left(0-40 \%\right.$ EtOAc / hexanes) provided $17(36 \mathrm{mg}, 60 \%)$ as a film. ${ }^{1} \mathrm{H}$ NMR $\left(400 \mathrm{MHz}, \mathrm{CDCl}_{3}\right): \delta 8.39(\mathrm{bs}, 1 \mathrm{H}), 6.82(\mathrm{~d}, 1 \mathrm{H}, J=8.8), 6.74(\mathrm{dd}, 1 \mathrm{H}, J=3.2,8.8), 6.56(\mathrm{~d}, 1 \mathrm{H}$, $J=3.2$ ), 5.92 (ddd, $1 \mathrm{H}, J=2.0,6.0,10.8), 5.85$ (ddd, $1 \mathrm{H}, J=1.2,2.8,10.0), 5.80(\mathrm{~m}, 1 \mathrm{H}), 5.01(\mathrm{~m}, 1 \mathrm{H})$, $4.47(\mathrm{~m}, 1 \mathrm{H}), 4.32$ (m, 1H), 3.81 (ddd, $2 \mathrm{H}, J=6.0,11.2,31.2), 3.72$ (t, $1 \mathrm{H}, J=4.4), 2.17$ (m, 2H), 0,91 
(s, 9H), 0.09 (s, 6H) ppm; ${ }^{13} \mathrm{C}$ NMR $\left(100 \mathrm{MHz}, \mathrm{CDCl}_{3}\right): \delta 152.8,149.3,134.0,128.1,125.2,124.1$, 118.0, 117.6, 117.4, 113.6, 75.2, 72.3, 63.8, 55.7, 37.3, 25.8, 18.3, -5.4 ppm; IR (neat): 3387, 2920, $1654,1390,1083 \mathrm{~cm}^{-1} ;[\alpha]_{\mathrm{D}}{ }^{23}=-210^{\circ}\left(\mathrm{c}=1.0 \mathrm{CH}_{2} \mathrm{Cl}_{2}\right)$.

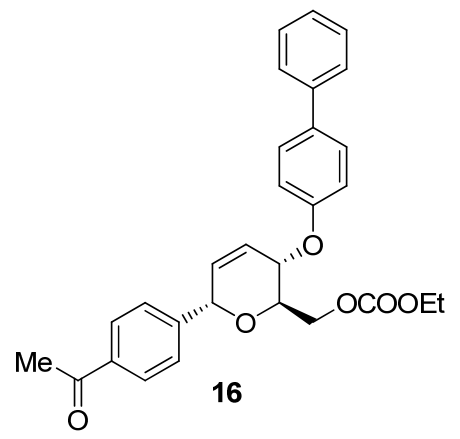

Aryl ether 16: Bis-carbonate SI-23 (200 mg, $0.50 \mathrm{mmol})$, (1S,2S)- (-)1,2- Diaminocyclohexane- N,N'-bis(2'-diphenylphosphinobenzoyl) (18 $\mathrm{mg}, \quad 0.013 \mathrm{mmol})$, and Tris(dibenzylideneacetone) dipalladium(0) chloroform adduct $(13 \mathrm{mg}, 0.025 \mathrm{mmol})$ were dissolved in degassed methylene chloride $(2.0 \mathrm{~mL})$ and stirred for $15 \mathrm{~min}$. The color of the solution changed from maroon to yellowish-orange. $p$-hydroxybiphenyl $(91 \mathrm{mg}, 0.54 \mathrm{mmol}$ ) was added and the reaction was irradiated in a microwave oven for $15 \min (150-300 \mathrm{~W}$, Powermax enabled) at $100^{\circ} \mathrm{C}$. The reaction mixture concentrated onto $\mathrm{SiO}_{2}$ and chromatographed over $\mathrm{SiO}_{2}$ $\left(0-50 \%\right.$ ethyl acetate / pet. ether) to provide $\mathbf{1 6}(170 \mathrm{mg}, 85 \%)$ as a viscous oil. ${ }^{1} \mathrm{H}$ NMR $(400 \mathrm{MHz}$, $\left.\mathrm{CDCl}_{3}\right): \delta 7.98(\mathrm{~d}, 2 \mathrm{H}, J=8.4), 7.56(\mathrm{~m}, 6 \mathrm{H}), 7.43(\mathrm{t}, 2 \mathrm{H}, J=7.2), 7.33(\mathrm{t}, 1 \mathrm{H}, J=8.4), 7.00(\mathrm{~d}, 2 \mathrm{H}$, $J=8.4$ ), 6.24 (bs, 2H), 5.43 (bs, $1 \mathrm{H}), 4.94$ (dd, $1 \mathrm{H}, J=2.0,8.4), 4.38$ (m, 2H), 4.17 (q, 2H, $J=7.2$, 14.4), $3.93(\mathrm{~m}, 1 \mathrm{H}), 2.62(\mathrm{~s}, 3 \mathrm{H}), 1.29(\mathrm{t}, 3 \mathrm{H}, J=6.8) \mathrm{ppm} ;{ }^{13} \mathrm{C} \mathrm{NMR}\left(100 \mathrm{MHz}, \mathrm{CDCl}_{3}\right): \delta 197.6$, $156.4,155.0,144.2,140.5,136.7,134.7,129.9,128.7,128.6,128.4,127.7,126.7,125.9,115.9$, 104.7, 73.6, 69.6, 68.2, 66.4, 64.2, 26.6, 14.2 ppm; IR (neat): 3037, 2982, 2900, 1743, 1677, 1607, $1487,1258,1009 \mathrm{~cm}^{-1}$; HRMS (Tof) $[\mathrm{M}+\mathrm{Na}]^{+}$: calcd. for $\mathrm{C}_{229} \mathrm{H}_{28} \mathrm{O}_{6} \mathrm{Na} 495.1784$, found 495.1798 . $[\alpha]_{\mathrm{D}}^{23}=+60^{\circ}\left(\mathrm{c}=1.0 \mathrm{CH}_{2} \mathrm{Cl}_{2}\right)$.

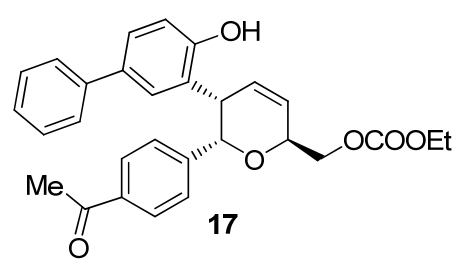

Phenol 17: Aryl ether $16(20 \mathrm{mg}, 0.04 \mathrm{mmol})$ was dissolved in chlorobenzene $(0.50 \mathrm{~mL})$ in a $10 \mathrm{~mL}$ microwave tube. $\mathrm{Eu}(\mathrm{fod})_{3}(44 \mathrm{mg}$, $0.04 \mathrm{mmol}$ ) was added and the reaction was heated with microwave irradiation $\left(300 \mathrm{~W}, 200^{\circ} \mathrm{C}\right)$ for $60 \mathrm{~min}$. Concentration in vacuo, followed by purification over $\mathrm{SiO}_{2}(0-80 \%$ EtOAc / hexanes $)$ provided $17(14 \mathrm{mg}, 70 \%)$ as a film. ${ }^{1} \mathrm{H}$ NMR $\left(400 \mathrm{MHz}, \mathrm{CDCl}_{3}\right): \delta 7.77(\mathrm{~d}, 2 \mathrm{H}$, $J=7.2$ ), 7.39 (m, 6H), 7.27 (m, 4H), 6.77 (d, $1 \mathrm{H}, J=8.0), 6.20$ (m, 1H), 5.97 (dq, $1 \mathrm{H}, J=1.6,3.2,10$ ), 5.45 (d, 1H, $J=3.6), 4.98$ (m, 1H), 4.64 (appq, $1 \mathrm{H}, J=8.0,12.0$ ), 4.22(dd, $1 \mathrm{H}, J=3.6,12.4), 4.14$ (q, $3 \mathrm{H}, J=7.2,14.4), 3.93(\mathrm{~m}, 1 \mathrm{H}), 2.49(\mathrm{~s}, 3 \mathrm{H}), 1.25(\mathrm{t}, 1 \mathrm{H}, J=7.6) \mathrm{ppm} ;{ }^{13} \mathrm{C} \mathrm{NMR}\left(100 \mathrm{MHz}, \mathrm{CDCl}_{3}\right)$ : $\delta$ 197.9, 155.0, 154.0, 144.0, $140.6,135.9,133.0,130.4,130.3,128.6,128.0,127.4,126.6,125.7$, 123.4, 123.1, 117.2, 73.3, 73.2, 66.0, 64.4, 26.5, $14.1 \mathrm{ppm}$; IR (neat): 3348, 2920, 1744, 1678, 1604, $1487,1405,1277,1107,998 \mathrm{~cm}^{-1}$; HRMS (Tof) $[\mathrm{M}+\mathrm{Na}]^{+}$: calcd. for $\mathrm{C}_{29} \mathrm{H}_{28} \mathrm{O}_{6} \mathrm{Na} 495.1784$, found 495.1787. $[\alpha]_{\mathrm{D}}^{23}=-228^{\circ}\left(\mathrm{c}=1.0 \mathrm{CH}_{2} \mathrm{Cl}_{2}\right)$.

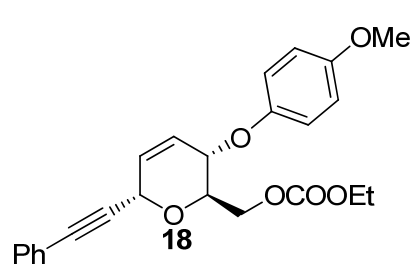

Aryl ether 18: Bis-carbonate SI-21 (569 mg. $1.52 \mathrm{mmol}),(1 S, 2 S)-(-)-$ 1,2- Diaminocyclohexane- N,N'-bis(2'-diphenylphosphinobenzoyl) (50 mg, $0.08 \mathrm{mmol}$ ), and Tris(dibenzylideneacetone)dipalladium(0) chloroform adduct (39 $\mathrm{mg}, 0.04 \mathrm{mmol}$ ) were dissolved in degassed methylene chloride $(4.0 \mathrm{~mL})$ and stirred for $15 \mathrm{~min}$. The color of the solution changed from 
maroon to yellowish-orange. $p$-Methoxyphenol $(198 \mathrm{mg}, 1.60 \mathrm{mmol})$ was added and the reaction was irradiated for $15 \mathrm{~min}\left(150-300 \mathrm{~W}\right.$, Powermax enabled) at $100^{\circ} \mathrm{C}$. Purification by $\mathrm{SiO}_{2}$ chromatography (0-40\% ethyl acetate / petroleum ether) provided 18 (583 mg, 94\%) as a viscous oil. ${ }^{1} \mathrm{H}$ NMR $\left(400 \mathrm{MHz}, \mathrm{CDCl}_{3}\right): \delta 7.46(\mathrm{~m}, 2 \mathrm{H}), 7.85(\mathrm{~m}, 4 \mathrm{H}), 6.01(\mathrm{dt}, 1 \mathrm{H}, J=1.2,10.4), 5.95(\mathrm{ddd}, 1 \mathrm{H}$, $J=1.6,3.2,10.4), 5.23$ (m, 1H), 4.77 (m, 1H), 4.53 (dd, 1H, $J=2.4,11.6), 4.39$ (dd, $1 \mathrm{H}, J=4.8,11.6)$, $4.29(\mathrm{~m}, 1 \mathrm{H}), 4.17$ (q, 2H, J=7.2), $3.77(\mathrm{~s}, 3 \mathrm{H}), 1.28(\mathrm{t}, 3 \mathrm{H}, J=7.2) \mathrm{ppm} ;{ }^{13} \mathrm{C} \mathrm{NMR}(100 \mathrm{MHz}$, $\left.\mathrm{CDCl}_{3}\right): \delta 155.0,154.5,150.9,131.8,128.6,128.5,128.3,125.6,122.2,117.1,114.8,86.6,84.9$, 70.6, 69.1, 66.5, 64.7, 64.1, 55.7, 14.2 ppm; IR (neat): 2959, 1743, 1503, 1444, 1382, 1266, 1227 , 1041, $827 \mathrm{~cm}^{-1}$; HRMS (Tof) $[\mathrm{M}+\mathrm{H}]^{+}$: calcd. for $\mathrm{C}_{224} \mathrm{H}_{25} \mathrm{O}_{6} \mathrm{~N}$ 409.1651, found 409.1654. $[\alpha]_{\mathrm{D}}{ }^{23}=$ $-27^{\circ}\left(\mathrm{c}=1.0 \mathrm{CH}_{2} \mathrm{Cl}_{2}\right)$.

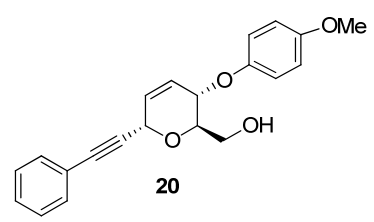

Aryl ether 20: Ethyl ester $18(337 \mathrm{mg}, 0.83 \mathrm{mmol})$ was dissolved in $\mathrm{MeOH}$ (5.0 mL). $\mathrm{K}_{2} \mathrm{CO}_{3}(28 \mathrm{mg}, 0.21 \mathrm{mmol})$ was added and the reaction was stirred for $2 \mathrm{~h}$. Purification over $\mathrm{SiO}_{2}(0-60 \%$ ethyl acetate / petroleum ether) provided $20(278 \mathrm{mg}, 100 \%)$ as a viscous oil. ${ }^{1} \mathrm{H}$ NMR $(400 \mathrm{MHz}$, $\left.\mathrm{CDCl}_{3}\right): \delta 7.47(\mathrm{~m}, 2 \mathrm{H}), 7.33(\mathrm{~m}, 3 \mathrm{H}), 6.91$ (appd, 2H, $\left.J=9.2\right), 6.83$ (appd, $2 \mathrm{H}, J=9.2), 6.02$ (dt, 1H, $J=1.6,10.4), 5.95$ (ddd, $1 \mathrm{H}, J=2.0,3.6,10.0), 5.23$ (m, 1H), 4.80 (dq, 1H, $J=1.6,8.8), 4.13(\mathrm{~m}, 1 \mathrm{H}), 4.00(\mathrm{~m}, 1 \mathrm{H}), 3.84(\mathrm{~m}, 1 \mathrm{H}), 3.77(\mathrm{~s}, 3 \mathrm{H}), 2.04(\mathrm{bs}, 1 \mathrm{H}) \mathrm{ppm} ;{ }^{13} \mathrm{C}$ NMR $\left(100 \mathrm{MHz}, \mathrm{CDCl}_{3}\right): \delta 154.4,151.3,131.9,128.7,128.3,128.2,126.2,122.2,117.2,114.8,86.4,85.1$, 72.7, 69.2, 64.6, 62.2, 55.7 ppm; IR (neat): 3464, 2935, 1507, 1219, 1087, 1037, 827, 761, $691 \mathrm{~cm}^{-1}$; HRMS (Tof) $[\mathrm{M}+\mathrm{H}]^{+}$: calcd. for $\mathrm{C}_{21} \mathrm{H}_{21} \mathrm{O}_{4} 337.1440$, found 337.1453. $[\alpha]_{\mathrm{D}}^{23}=+6^{\mathrm{o}}\left(\mathrm{c}=1.0 \mathrm{CH}_{2} \mathrm{Cl}_{2}\right)$.

\section{Gold(III)- catalyzed ring contraction of $\boldsymbol{C}$-glycosides}
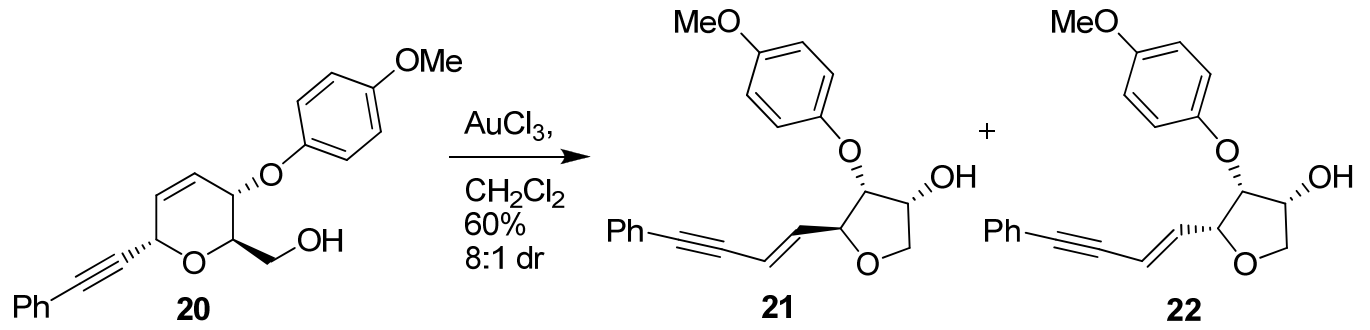

Procedure: 20 (7 mg, $0.02 \mathrm{mmol})$ was dissolved in $\mathrm{CH}_{2} \mathrm{Cl}_{2}(1.0 \mathrm{~mL})$ under argon. $\mathrm{AuCl}_{3}(0.6 \mathrm{mg}$, $0.002 \mathrm{mmol}$ ) was added. After 12 hours the reaction was concentrated onto $\mathrm{SiO}_{2}$ and chromatographed over $\mathrm{SiO}_{2}(0-80 \%$ ethyl acetate /pet. ether) to provide diastereomers 21 (major), 22 (minor).

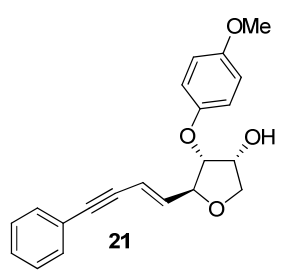

Ene-yne 21: $3.7 \mathrm{mg}(53 \%) .{ }^{1} \mathrm{H}$ NMR (400 $\left.\mathrm{MHz}, \mathrm{CDCl}_{3}\right): \delta 7.43(\mathrm{~m}, 2 \mathrm{H})$, $7.312(\mathrm{~m}, 3 \mathrm{H}), 6.92(\mathrm{~d}, 2 \mathrm{H}, J=8.8), 6.86(\mathrm{~d}, 2 \mathrm{H}), J=6.8), 6.23(\mathrm{dd}, 1 \mathrm{H}, J=6.0$, 15.6), 6.05 (dd, 1H, $J=1.6,15.6), 4.57(\mathrm{td}, 1 \mathrm{H}, J=1.6,6.0), 4.51$ (q, 1H, $J=5.2)$, 4.34 (t, 1H, $J=5.6), 4.23$ (dd, 1H, $J=5.2,9.6), 3.92$ (dd, 1H, $J=4.4,9.2), 3.78$ (s, $3 \mathrm{H}) \mathrm{ppm} ;{ }^{13} \mathrm{C} \mathrm{NMR}\left(100 \mathrm{MHz}, \mathrm{CDCl}_{3}\right): \delta 155.1,151.1,139.7,131.5,128.4,128.3$, $123.0,117.4,114.9,112.1,90.9,87.0,83.0,80.5,72.9,70.2,55.7$ ppm; IR (neat): 
3441, 2948, 1499, 1445, 1227, $1037 \mathrm{~cm}^{-1}$; HRMS (Tof) $[\mathrm{M}+\mathrm{H}]^{+}$: calcd. for $\mathrm{C}_{21} \mathrm{H}_{21} \mathrm{O}_{4} \mathrm{~N} 337.1440$, found 337.1410. $[\alpha]_{\mathrm{D}}^{23}=-118^{\mathrm{o}}\left(\mathrm{c}=1.0 \mathrm{CH}_{2} \mathrm{Cl}_{2}\right)$.

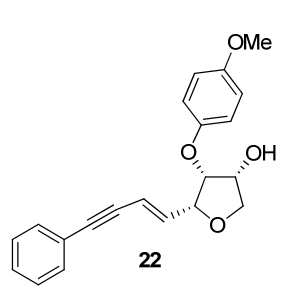

Ene-yne 22: $\quad 0.5 \mathrm{mg}(7 \%) . \quad{ }^{1} \mathrm{H} \mathrm{NMR}\left(400 \mathrm{MHz}, \mathrm{CDCl}_{3}\right): \delta 7.42(\mathrm{~m}, 2 \mathrm{H}), 7.31$ (m, 3H), 6.95 (d, 2H, $J=9.2), 6.84$ (d, 2H, $J=9.2), 6.35$ (dd, 1H, $J=6.4,16.0), 5.99$ $(\mathrm{dd}, 1 \mathrm{H}, J=1.2,15.6), 4.67(\mathrm{~m}, 2 \mathrm{H}), 4.54(\mathrm{~m}, 1 \mathrm{H}), 4.06$ (dd, $1 \mathrm{H}, J=5.2,9.2), 3.99$ $(\mathrm{dd}, 1 \mathrm{H}, J=4.4,10.0), 3.78(\mathrm{~s}, 3 \mathrm{H}) \mathrm{ppm} ;{ }^{13} \mathrm{C} \mathrm{NMR}\left(100 \mathrm{MHz}, \mathrm{CDCl}_{3}\right): \delta 154.9$, $152.0,138.5,131.5,128.3,128.2,123.2,117.4,114.8,112.7,90.6,87.3,81.0,79.8$, 72.6, 71.7, $55.7 \mathrm{ppm}$.

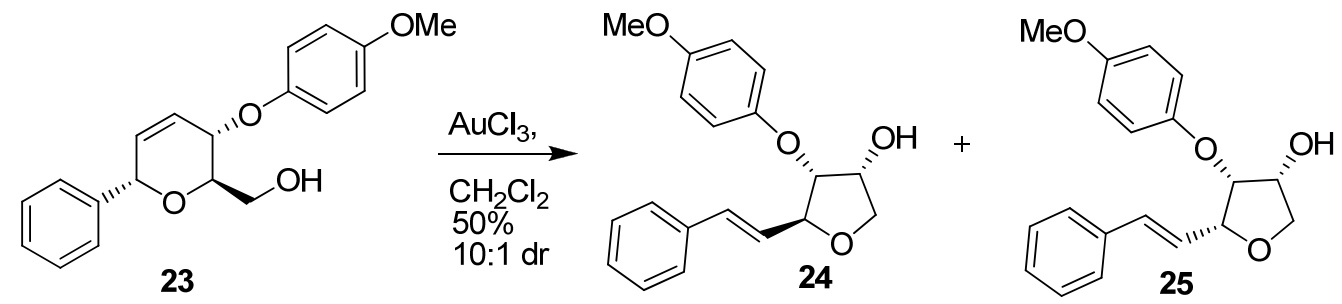

Procedure: 23 (86 mg, $0.3 \mathrm{mmol})$ was dissolved in $\mathrm{CH}_{2} \mathrm{Cl}_{2}(1.0 \mathrm{~mL})$ under argon. $\mathrm{AuCl}_{3}(20 \mathrm{mg}$, $0.08 \mathrm{mmol}$ ) was added. After 8 hours the reaction was concentrated onto $\mathrm{SiO}_{2}$ and chromatographed over $\mathrm{SiO}_{2}(0-80 \%$ ethyl acetate /pet. ether) to provide diastereomers 24 (major), 25 (minor).

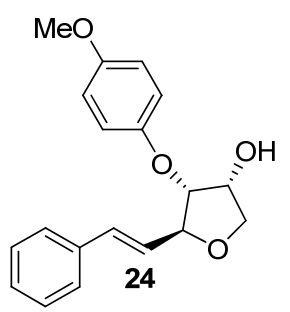

Tetrahydrofuran 24: $39 \mathrm{mg}(45 \%) .{ }^{1} \mathrm{H} \mathrm{NMR}\left(400 \mathrm{MHz}, \mathrm{CDCl}_{3}\right): \delta 7.32(\mathrm{~m}$, $5 \mathrm{H}), 6.93(\mathrm{~d}, 2 \mathrm{H}, J=9.6), 6.83(\mathrm{~d}, 2 \mathrm{H}, J=9.2), 6.71(\mathrm{~d}, 1 \mathrm{H}, J=16.0), 6.219 \mathrm{dd}, 1 \mathrm{H}$, $J=7.2,16.0), 4.64(\mathrm{t}, 1 \mathrm{H}, J=6.0), 4.53(\operatorname{appd}, 1 \mathrm{H}, J=4.89), 4.38(\mathrm{t}, 1 \mathrm{H}, J=5.2)$, $4.27(\mathrm{dd}, 1 \mathrm{H}, J=5.6,10.0), 3.93(\mathrm{dd}, 1 \mathrm{H}, J=4.4,9.6), 3.77(\mathrm{~s}, 3 \mathrm{H}), 2.59(\mathrm{~s},-\mathrm{OH})$ ppm; ${ }^{13} \mathrm{C}$ NMR $\left(100 \mathrm{MHz}, \mathrm{CDCl}_{3}\right): \delta 155.0,151.3,132.7,128.6,127.9,126.6$, 126.5, 117.4, 114.8, 83.2, 81.2, 72.8, 70.3, 55.7 ppm; IR (neat): 3351, 2959, 1643, 1499, 1262, 1219, $1033 \mathrm{~cm}^{-1}$; HRMS (Tof) $[\mathrm{M}+\mathrm{Na}]^{+}$: calcd. for $\mathrm{C}_{19} \mathrm{H}_{20} \mathrm{O}_{4} \mathrm{Na} 335.1259$, found 335.1243. $[\alpha]_{\mathrm{D}}{ }^{23}=+60^{\circ}\left(\mathrm{c}=1.0 \mathrm{CH}_{2} \mathrm{Cl}_{2}\right)$.

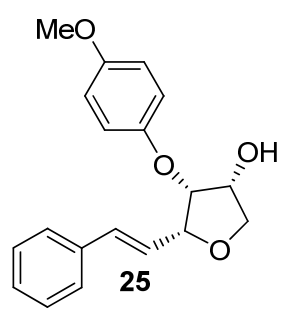

Tetrahydrofuran 25: $4 \mathrm{mg}(5 \%) . \quad{ }^{1} \mathrm{H}$ NMR (400 MHz, $\left.\mathrm{CDCl}_{3}\right): 7.28(\mathrm{~m}, 5 \mathrm{H})$, $6.92(\mathrm{~d}, 2 \mathrm{H}, J=9.2), 6.79(\mathrm{~d}, 2 \mathrm{H}, J=9.2), 6.64(\mathrm{~d}, 1 \mathrm{H}, J=15.6), 6.35$ (dd, $1 \mathrm{H}, J=7.2$, 16.4), $4.69(\mathrm{~m}, 1 \mathrm{H}), 4.59(\mathrm{~m}, 1 \mathrm{H}), 4.03(\mathrm{dd}, 1 \mathrm{H}, J=6.0,9.2), 3.99(\mathrm{dd}, 1 \mathrm{H}, J=4.8$, 9.2), $3.76(\mathrm{~s}, 3 \mathrm{H}), 2.63(\mathrm{~d}, 1 \mathrm{H}, J=7.2(-\mathrm{OH})) 155.0,151.3,136.3,132.7,128.6$, $127.9,126.6,126.5,117.5,114.8,83.2,81.2,72.8,70.3,55.7 \mathrm{ppm} ;{ }^{13} \mathrm{C} \mathrm{NMR}(100$ $\left.\mathrm{MHz}, \mathrm{CDCl}_{3}\right): \delta 135.6,132.7,126.0,122.7,115.9,93.8,92.5,67.0,66.1,61.4$, 60.9, 54.1, 49.3, 25.7 24.3, 18.0, -4.6, -4.8 ppm; IR (neat): 3436, 1506, 1234, 1041, $753 \mathrm{~cm}^{-1}$; HRMS (Tof) $[\mathrm{M}+\mathrm{Na}]^{+}$: calcd. for $\mathrm{C}_{19} \mathrm{H}_{20} \mathrm{O}_{4} \mathrm{Na} 335.1259$, found 335.1229. $[\alpha]_{\mathrm{D}}^{23}=-78^{\circ}\left(\mathrm{c}=1.0 \mathrm{CH}_{2} \mathrm{Cl}_{2}\right)$. 


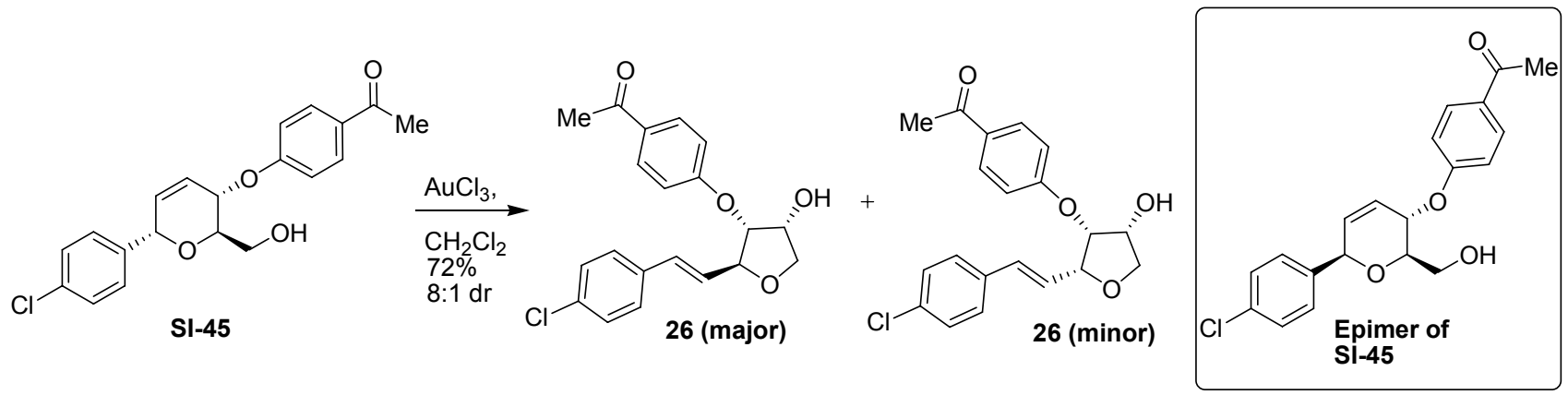

Procedure: SI-45 (25 mg, $0.1 \mathrm{mmol})$ was dissolved in $\mathrm{CH}_{2} \mathrm{Cl}_{2}(0.5 \mathrm{~mL})$ under argon. $\mathrm{AuCl}_{3}(6 \mathrm{mg}$, $0.02 \mathrm{mmol})$ was added. After 6 hours the reaction was concentrated onto $\mathrm{SiO}_{2}$ and chromatographed over $\mathrm{SiO}_{2}(0-80 \%$ ethyl acetate /pet. ether) to provide diastereomers $\mathbf{2 6}$ (major), $\mathbf{2 6}$ (minor) and the epimer of SI-45.

Tetrahydrofuran 26 (major): $15.9 \mathrm{mg}(64 \%) .{ }^{1} \mathrm{H} \mathrm{NMR}\left(400 \mathrm{MHz}, \mathrm{CDCl}_{3}\right):$
$\delta 7.95(\mathrm{~d}, 2 \mathrm{H}, J=9.2), 7.3(\mathrm{~m}, 4 \mathrm{H}), 7.02(\mathrm{~d}, 2 \mathrm{H}, J=9.2), 6.68(\mathrm{~d}, 1 \mathrm{H}, J=15.6)$,
$6.20(\mathrm{dd}, 1 \mathrm{H}, J=6.8,16.0), 4.62(\mathrm{~m}, 2 \mathrm{H}), 4.33(\mathrm{dd}, 1 \mathrm{H}, J=5.2,9.6), 3.93(\mathrm{dd}, 1 \mathrm{H}$, HRMS (Tof) $[\mathrm{M}+\mathrm{H}]^{+}$: calcd. for $\mathrm{C}_{20} \mathrm{H}_{20} \mathrm{O}_{4} \mathrm{Cl} 359.1050$, found 359.1052. $[\alpha]_{\mathrm{D}}{ }^{23}=+52^{\circ}(\mathrm{c}=1.0$ $\mathrm{CH}_{2} \mathrm{Cl}_{2}$ ).

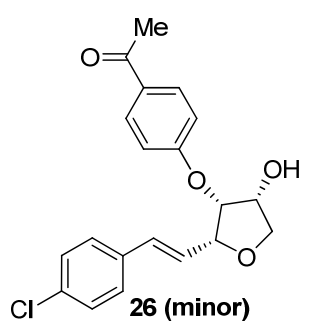

Tetrahydrofuran 26 (minor): $2 \mathrm{mg}(8 \%) .{ }^{1} \mathrm{H}$ NMR $\left(400 \mathrm{MHz}, \mathrm{CDCl}_{3}\right): \delta$ 7.91 (d, 2H, $J=8.4), 7.3(\mathrm{~m}, 4 \mathrm{H}), 7.01$ (d, 2H, $J=8.0), 6.56$ (d, $1 \mathrm{H}, J=16.0), 6.23$ (dd, $1 \mathrm{H}, J=7.2,16.0), 4.92(\mathrm{t}, 1 \mathrm{H}, J=4.8), 4.76$ (t, $1 \mathrm{H}, J=7.2), 4.6(\mathrm{t}, 1 \mathrm{H}, J=4.4)$, 4.12 (dd, $1 \mathrm{H}, J=6.0,10.0), 4.02(\mathrm{dd}, 1 \mathrm{H}, J=4.8,10.0), 2.56$ (s, $3 \mathrm{H}), 2.38$ (d, $1 \mathrm{H}$, $J=6.8(-\mathrm{OH})) \mathrm{ppm} ;{ }^{13} \mathrm{C}$ NMR $\left(100 \mathrm{MHz}, \mathrm{CDCl}_{3}\right): \delta 186.7,174.2,152.7,151.6$, $140.3,134.9,132.8,130.9,128.9,128.0,152.5,115.7,104.2,80.7,79.5,77.9$, 77.6, 72.3, 72.0, 26.4 ppm; IR (neat): 3401, 2924, 1673, 1603, 1502, 1405, 1254, $1095 \mathrm{~cm}^{-1} ;[\alpha]_{\mathrm{D}}^{23}=+52^{\circ}\left(\mathrm{c}=1.0 \mathrm{CH}_{2} \mathrm{Cl}_{2}\right)$.

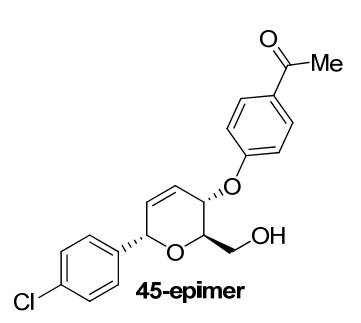

Tetrahydropyran 26 (epimer of 45): $6.5 \mathrm{mg}(26 \%) . \quad{ }^{1} \mathrm{H}$ NMR $(400 \mathrm{MHz}$, $\left.\mathrm{CDCl}_{3}\right): \delta 7.97(\mathrm{~d}, 2 \mathrm{H}, J=6.8), 7.31(\mathrm{~m}, 4 \mathrm{H}), 7.01(\mathrm{~d}, 2 \mathrm{H}, J=7.2), 6.04(\mathrm{dt}, \mathrm{H}$, $J=2.4,10.0), 5.93$ (dt, $1 \mathrm{H}, J=1.6,10.4), 5.08$ (dt, $1 \mathrm{H}, J=1.6,8.4), 3.93(\mathrm{~m}, 2 \mathrm{H})$, $3.79(\mathrm{~m}, 1 \mathrm{H}), 2.57(\mathrm{~s}, 3 \mathrm{H}), 1.99(\mathrm{~s}, 1 \mathrm{H},-\mathrm{OH}) \mathrm{ppm} ;{ }^{13} \mathrm{C} \mathrm{NMR}(100 \mathrm{MHz}$, $\left.\mathrm{CDCl}_{3}\right): \delta 196.7,161.2,138.3,134.3,132.3,130.9,130.9,130.8,128.9,128.7$, 124.9, 115.1, 77.6, 68.1, 62.2, $26.4 \mathrm{ppm}$; HRMS (Tof) $[\mathrm{M}+\mathrm{H}]^{+}$: calcd. for $\mathrm{C}_{20} \mathrm{H}_{20} \mathrm{O}_{4} \mathrm{Cl} 359.1050$, found 359.1000. IR (neat): 3374, 2916, 1669, 1600, 1499, 1351, 1243, 1165, $1091 \mathrm{~cm}^{-1} ;[\alpha]_{\mathrm{D}}^{23}=+140^{\circ}\left(\mathrm{c}=1.0 \mathrm{CH}_{2} \mathrm{Cl}_{2}\right)$. 


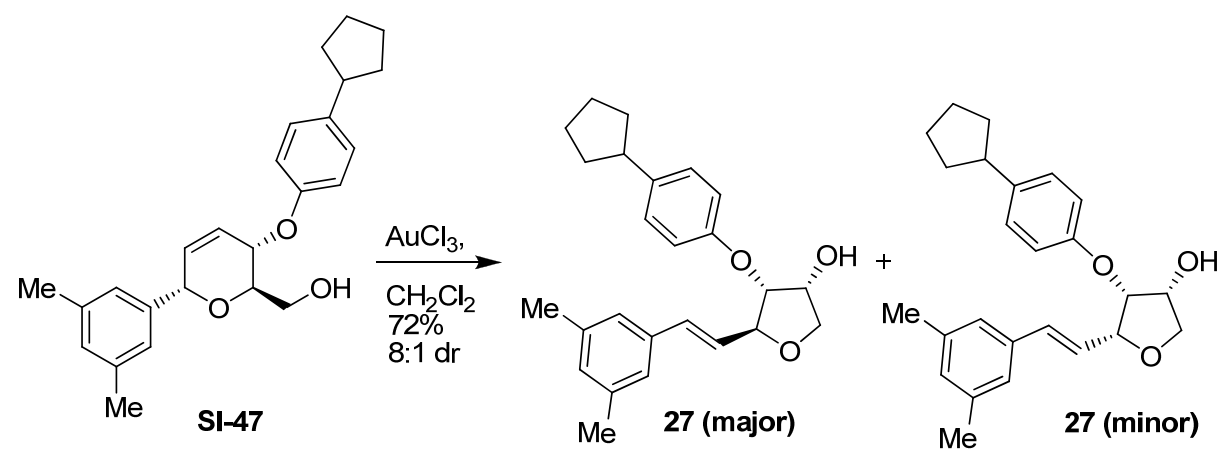

Procedure: SI-47 (42 mg, $0.1 \mathrm{mmol})$ was dissolved in $\mathrm{CH}_{2} \mathrm{Cl}_{2}(1.0 \mathrm{~mL})$ under argon. $\mathrm{AuCl}_{3}(5 \mathrm{mg}$, $0.02 \mathrm{mmol})$ was added. After 2 hours the reaction was concentrated onto $\mathrm{SiO}_{2}$ and chromatographed over $\mathrm{SiO}_{2}(0-80 \%$ ethyl acetate /pet. ether) to provide diastereomers 27 (major), 27 (minor).

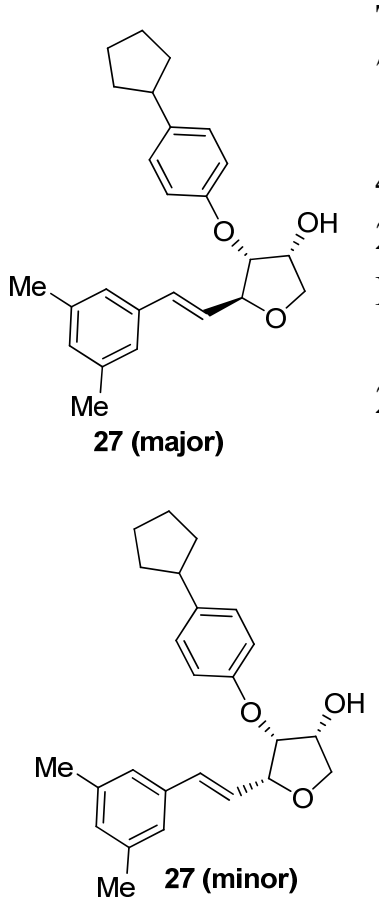

Tetrahydrofuran 27 (major): $14.5 \mathrm{mg}(35 \%) .{ }^{1} \mathrm{H}$ NMR $\left(400 \mathrm{MHz}, \mathrm{CDCl}_{3}\right)$ : 7.17 (d, 2H, $J=8.0), 7.00$ (s, 2H), 6.9 (m, 3H), 6.65 (d, 1H, $J=15.6), 6.19$ (dd, $1 \mathrm{H}, J=6.4,15.6), 4.64$ (t, $1 \mathrm{H}, J=5.6), 4.55$ (t, $1 \mathrm{H}, J=4.8), 4.43$ (t, $1 \mathrm{H}, J=5.2)$, 4.28 (dd, $1 \mathrm{H}, J=5.6,9.6), 3.92$ (dd, $1 \mathrm{H}, J=4.8,9.6) 2.94(\mathrm{~m}, 1 \mathrm{H}), 2.30$ (s, $6 \mathrm{H})$, $2.05(\mathrm{~m}, 2 \mathrm{H}), 1.81(\mathrm{~m}, 2 \mathrm{H}), 1.68(\mathrm{~m}, 2 \mathrm{H}), 1.54(\mathrm{~m}, 2 \mathrm{H}) \mathrm{ppm} ;{ }^{13} \mathrm{C}$ NMR $(100$ $\left.\mathrm{MHz}, \mathrm{CDCl}_{3}\right): \delta 155.3,140.4,138.0,136.2,132.9,129.7,128.3,126.0,124.5$, 115.8, 82.2, 81.4, 72.8, 70.3, 45.1, 34.7, 25.4, $21.2 \mathrm{ppm}$; IR (neat): 3413, 2955, $2850,1615,1507,144,1238,1099 \mathrm{~cm}^{-1} ;[\alpha]_{\mathrm{D}}^{23}=-75^{\circ}\left(\mathrm{c}=1.0 \mathrm{CH}_{2} \mathrm{Cl}_{2}\right)$.

Tetrahydrofuran 27 (minor): $5.6 \mathrm{mg}(13 \%) . \quad{ }^{1} \mathrm{H}$ NMR (400 $\mathrm{MHz}, \mathrm{CDCl}_{3}$ ): $\delta 7.13(\mathrm{~d}, 2 \mathrm{H}, J=8.0), 6.91(\mathrm{~m}, 5 \mathrm{H}), 6.56(\mathrm{~d}, 1 \mathrm{H}, J=15.6), 6.29(\mathrm{dd}, 1 \mathrm{H}$, $J=7.6,16.0), 4.76(\mathrm{t}, 1 \mathrm{H}, J=5.2), 4.69$ (m, $1 \mathrm{H}), 4.60(\mathrm{t}, 1 \mathrm{H}, J=4.8), 4.08$ (dd, $1 \mathrm{H}, J=6.0,9.6), 3.99$ (dd, 1H, $J=4.8,9.2), 2.92(\mathrm{~m}, 1 \mathrm{H}), 2.26(\mathrm{~s}, 6 \mathrm{H}), 2.02$ $(\mathrm{m}, 2 \mathrm{H}), 1.78(\mathrm{~m}, 2 \mathrm{H}), 1.67(\mathrm{~m}, 2 \mathrm{H}), 1.56(\mathrm{~m}, 2 \mathrm{H}) \mathrm{ppm} ;{ }^{13} \mathrm{C} \mathrm{NMR}(100 \mathrm{MHz}$, $\left.\mathrm{CDCl}_{3}\right): \delta 139.9,137.9,133.6,129.5,128.1,124.8,124.6,116.0,81.3,80.3$, 77.9, 72.5, 72., 49.3, 45.1, 34.7, 25.4, 21.22 ppm; IR (neat3371, 2959, 2862, $1720,1603,1507,1238,1102,1037 \mathrm{~cm}^{-1} ;[\alpha]_{\mathrm{D}}^{23}=+21^{\circ}\left(\mathrm{c}=1.0 \mathrm{CH}_{2} \mathrm{Cl}_{2}\right)$. 


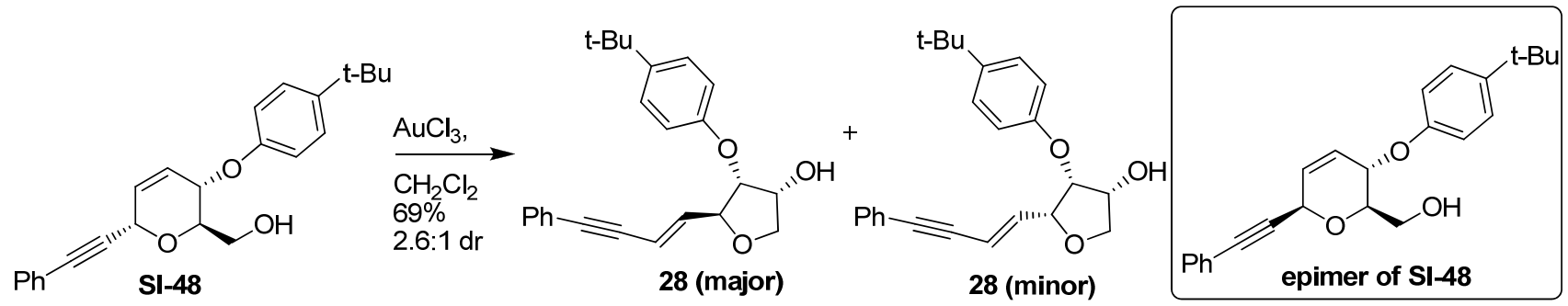

Procedure: SI-48 (71 mg, $0.2 \mathrm{mmol})$ was dissolved in $\mathrm{CH}_{2} \mathrm{Cl}_{2}(0.5 \mathrm{~mL})$ under argon. $\mathrm{AuCl}_{3}(18 \mathrm{mg}$, $0.06 \mathrm{mmol}$ ) was added. After 5 hours the reaction was concentrated onto $\mathrm{SiO}_{2}$ and chromatographed over $\mathrm{SiO}_{2}(0-80 \%$ ethyl acetate /pet. ether) to provide diastereomers 28 (major), 28 (minor) and the epimer of SI-48.
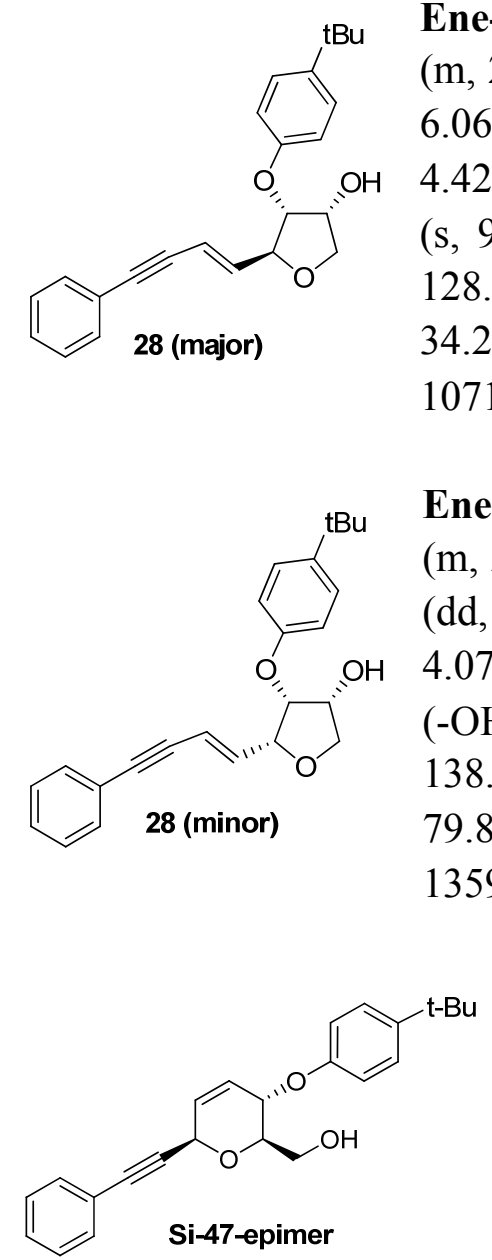

Ene-yne 28 (major): $37 \mathrm{mg}(52 \%) .{ }^{1} \mathrm{H}$ NMR (400 MHz, $\left.\mathrm{CDCl}_{3}\right): \delta 7.43$ (m, 2H), 7.33 (m, 5H), 6.91 (dd, 2H, $J=2.4,7.2), 6.25$ (dd, $1 \mathrm{H}, J=5.6,15.6$ ), 6.06 (dd, $1 \mathrm{H}, J=1.2,16.0$ ), 4.58 (dt, $1 \mathrm{H}, J=1.2,6.0$ ), 4.54 (q, $1 \mathrm{H}, J=4.8$ ), 4.42 (t, $1 \mathrm{H}, J=5.6$ ), 4.25 (dd, $1 \mathrm{H}, J=5.6,10.0), 3.93$ (dd, $1 \mathrm{H}, J=4.8,9.6) 1.3$ (s, 9H) ppm; ${ }^{13} \mathrm{C}$ NMR $\left(100 \mathrm{MHz}, \mathrm{CDCl}_{3}\right): \delta 154.8,145.3,139.6,131.5$, 128.3, 126.6, 126.4, 123.0, 115.4, 112.2, 90.9, 87.0, 81.9, 80.5, 72.9, 70.2, 34.2, 31.4 ppm; IR (neat): 3440, 3045, 2963, 2866, 1600, 1511, 1363, 1227, $1071 \mathrm{~cm}^{-1} ;[\alpha]_{\mathrm{D}}^{23}=-100^{\circ}\left(\mathrm{c}=1.0 \mathrm{CH}_{2} \mathrm{Cl}_{2}\right)$.

Ene-yne 28 (minor): $14 \mathrm{mg}(20 \%) .{ }^{1} \mathrm{H}$ NMR (400 MHz, $\left.\mathrm{CDCl}_{3}\right): \delta 7.42$ (m, 2H0, $7.31(\mathrm{~m}, 5 \mathrm{H}), 6.94$ (d, 2H, $J=8.4), 6.35$ (dd, $1 \mathrm{H}, J=6.8,16.0), 6.00$ (dd, $1 \mathrm{H}, J=1.2,15.6), 4.77$ (t, 1H, $J=5.2), 4.67$ (t, $1 \mathrm{H}, J=6.4), 4.55(\mathrm{~m}, 1 \mathrm{H})$, 4.07 (dd, $1 \mathrm{H}, J=5.6,10.0), 4.00(\mathrm{dd}, 1 \mathrm{H}, J=3.6,9.6), 2.52$ (d, $1 \mathrm{H}, J=6.0$ (-OH), 1.30 (s, 9H) ppm; ${ }^{13} \mathrm{C}$ NMR $\left(100 \mathrm{MHz}, \mathrm{CDCl}_{3}\right): \delta$ 155.6, 145.2, 138.4, 131.5, 128.7, 128.4, 128.3, 128.2, 126.5, 115.6, 112.7, 90.6, 87.4, 80.1, 79.8, 72.6, 71.7, 34.2, 31.4 ppm; IR (neat): 3440, 2963, 1685, 1600, 1503, $1359,1255,1184 \mathrm{~cm}^{-1} ;[\alpha]_{\mathrm{D}}^{23}=+110^{\circ}\left(\mathrm{c}=1.0 \mathrm{CH}_{2} \mathrm{Cl}_{2}\right)$.

Tetrahydropyran 28 (epimer of SI-47): $9 \mathrm{mg}(13 \%) .{ }^{1} \mathrm{H}$ NMR (400 $\left.\mathrm{MHz}, \mathrm{CDCl}_{3}\right): \delta 7.47(\mathrm{~m}, 2 \mathrm{H}), 7.33(\mathrm{~m}, 5 \mathrm{H}), 6.89(\mathrm{~d}, 2 \mathrm{H}, J=8.8), 6.04$ $(\mathrm{d}, 1 \mathrm{H}, J=10.0), 5.95(\mathrm{dq}, 1 \mathrm{H}, J=1.6,3.2,10.0), 5.23(\mathrm{~m}, 1 \mathrm{H}), 4.88$ (dd, $1 \mathrm{H}, J=2.0,9.2), 4.15(\mathrm{~m}, 1 \mathrm{H}), 3.9(\mathrm{~m}, 1 \mathrm{H}), 3.82(\mathrm{~m}, 1 \mathrm{H}), 1.30(\mathrm{~s}$, 9H) $\mathrm{ppm} ;{ }^{13} \mathrm{C} \mathrm{NMR}\left(100 \mathrm{MHz}, \mathrm{CDCl}_{3}\right): \delta 154.9,144.2,131.9,128.7$, $128.3,128.3,126.5,126.2,122.2,115.2,86.4,85.1,72.7,68.2,64.6$, 62.3, 34.1, 61.5 ppm; IR (neat): 3378, 2959, 1705, 1611, 1506, 1371, $1254,1180,1067 \mathrm{~cm}^{-1} ;[\alpha]_{\mathrm{D}}^{23}=+19^{\circ}\left(\mathrm{c}=1.0 \mathrm{CH}_{2} \mathrm{Cl}_{2}\right)$. 

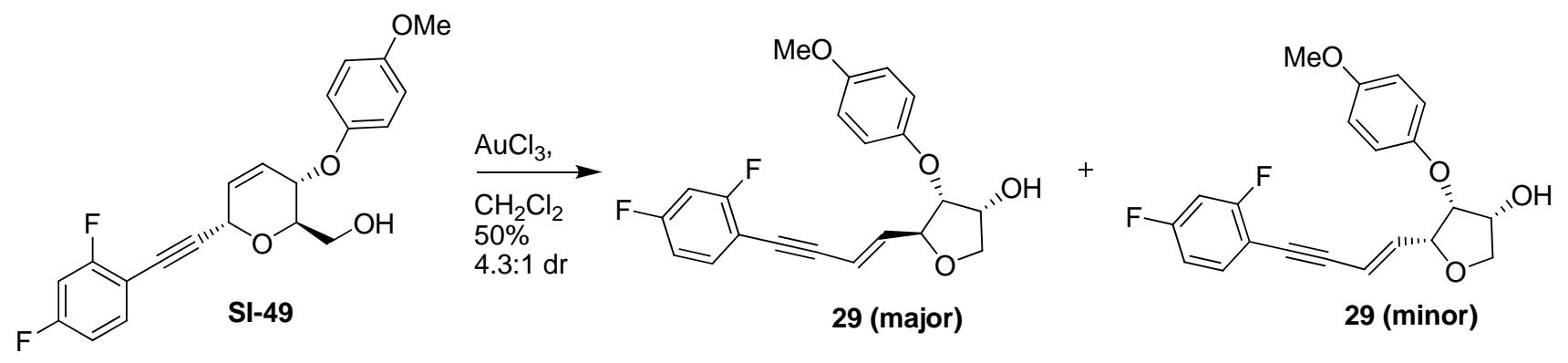

Procedure: SI-49 (46 mg, $0.12 \mathrm{mmol})$ was dissolved in $\mathrm{CH}_{2} \mathrm{Cl}_{2}(1.0 \mathrm{~mL})$ under argon. $\mathrm{AuCl}_{3}(6 \mathrm{mg}$, $0.02 \mathrm{mmol}$ ) was added. After 2 hours the reaction was concentrated onto $\mathrm{SiO}_{2}$ and chromatographed over $\mathrm{SiO}_{2}$ (0-80\% ethyl acetate /pet. ether) to provide diastereomers 29(major), 29 (minor).

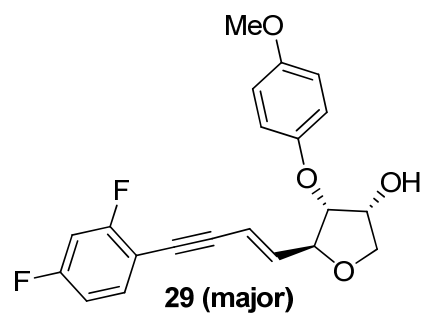

Ene-yne 29 (major): $18.7 \mathrm{mg}$ (42\%). ${ }^{1} \mathrm{H}$ NMR (400 $\left.\mathrm{MHz}, \mathrm{CDCl}_{3}\right): \delta$ 7.39 (m, 1H), 6.87 (m, 6H), 6.27 (dd, 1H, J=6.0, 15.6), 6.06 (dd, 1H, $J=1.6,16.0$ ), 4.58 (t, 1H, J=5.2), 4.49 (m, 1H0, 4.33 (t, 1H, $J=5.2), 4.23$ (dd, $1 \mathrm{H}, J=4.8,9.2$ ), 3.92 (dd, 1H, $J=4.4,9.2$ ), 3.78 (s, 3H0, 2.52 (d, 1H, $J=5.6(-\mathrm{OH})) \mathrm{ppm} ;{ }^{13} \mathrm{C}$ NMR $\left(100 \mathrm{MHz}, \mathrm{CDCl}_{3}\right): \delta 164.0(\mathrm{t}, 1 \mathrm{C}, J=38.0)$, 161.6 (t, 1C, $J=50.0$ ), 155.1, 151.1, 140.6, 134.2 (dd, 1C, $J=11.6,49.6$ ), 117.4, 114.9. 111.7 (t, 1C, $J=14.4$ ), 107.9, 104.3 (t, 1C, $J=102.8), 91.8$, 83.1, 82.9, 80.4, 77.9, 72.9, 70.3, 55.7 ppm; HRMS (Tof) $[\mathrm{M}+\mathrm{Na}]^{+}$: calcd. for $\mathrm{C}_{21} \mathrm{H}_{18} \mathrm{O}_{4} \mathrm{NaF}_{2}$ 395.1071, found 395.1060. IR (neat): 3436, 2932, 1619, 1514, 1464, 1425, 1266, 1227, 1141, 1095, $959 \mathrm{~cm}^{-1} ;[\alpha]_{\mathrm{D}}^{23}=-112^{\circ}\left(\mathrm{c}=1.0 \mathrm{CH}_{2} \mathrm{Cl}_{2}\right)$.

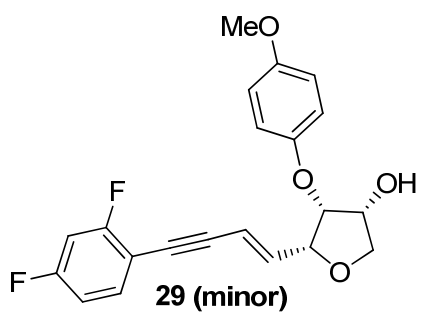

Ene-yne 29 (minor): $4.3 \mathrm{mg}(9 \%) . \quad{ }^{1} \mathrm{H} \mathrm{NMR}\left(400 \mathrm{MHz}, \mathrm{CDCl}_{3}\right): \delta 7.44$ (m, 1H), 6.94 (m, 2H), 6.83 (m, 4H), 6.38 (dd, 1H, $J=6.0,15.6), 6.00$ (dd, $1 \mathrm{H}, J=1.2,15.6), 4.67$ (m, 2H), 4.55 (m, 1H), 4.06 (dd, $1 \mathrm{H}, J=5.2,10.0$ ), 3.99 (dd, $1 \mathrm{H}, J=4.4,9.6), 3.77$ (s, 3H), 2.53 (d, 1H, $J=6.0(-\mathrm{OH})$ ) ppm; ${ }^{13} \mathrm{C}$ NMR (100 MHz, $\left.\mathrm{CDCl}_{3}\right): \delta 154.9,151.9,139.5,134.2,117.4,114.8$, $112.1,111.6,111.4,104.2,81.0,79.6,77.7,726,71.6,55.7$ ppm; IR (neat): 3425, 2959, 2920, 1716, 1615, 1506, 1429, 1262, 1223, 1099, $1040 \mathrm{~cm}^{-1}$; $\quad$ HRMS (Tof) $[\mathrm{M}+\mathrm{Na}]^{+}$: calcd. for $\mathrm{C}_{21} \mathrm{H}_{18} \mathrm{O}_{4} \mathrm{NaF}_{2}$ 395.1071, found 395.1067. $[\alpha]_{\mathrm{D}}^{23}=$ $+121.8^{\circ}\left(\mathrm{c}=1.0 \mathrm{CH}_{2} \mathrm{Cl}_{2}\right)$. 

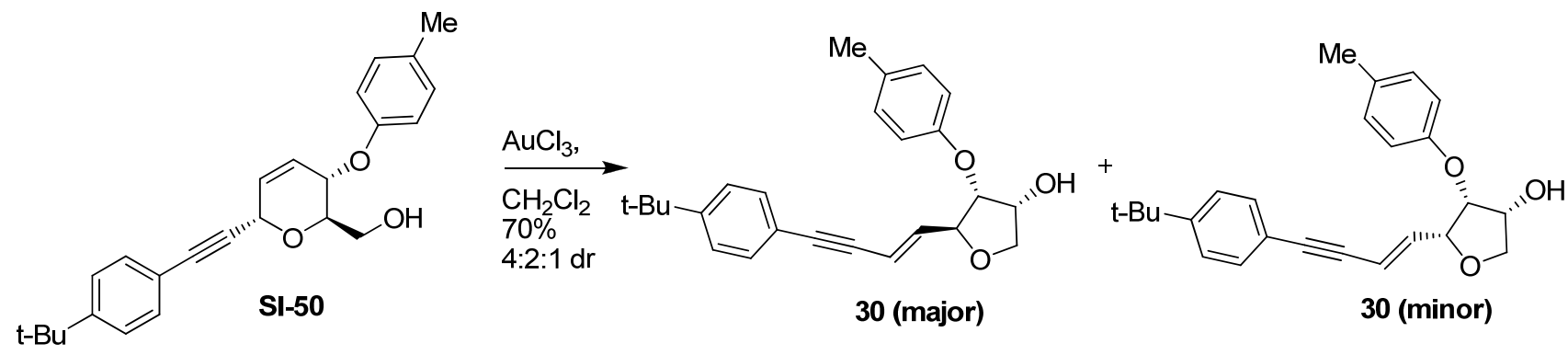

Procedure: SI-50 (40 mg, $0.1 \mathrm{mmol}$ ) was dissolved in $\mathrm{CH}_{2} \mathrm{Cl}_{2}(0.5 \mathrm{~mL})$ under argon. $\mathrm{AuCl}_{3}(6 \mathrm{mg}$, $0.02 \mathrm{mmol})$ was added. After 15 minutes the reaction was concentrated onto $\mathrm{SiO}_{2}$ and chromatographed over $\mathrm{SiO}_{2}$ (0-80\% ethyl acetate /pet. ether) to provide diastereomers 30(major), 30 (minor).

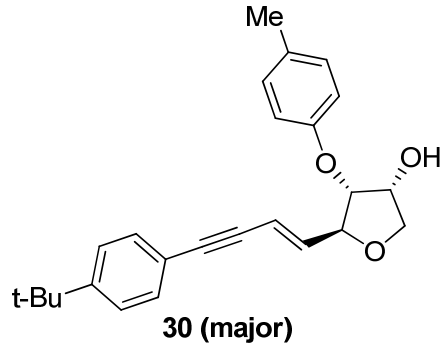

Ene-yne 30 (major): $22 \mathrm{mg}$ (56\%). Procedure ${ }^{1} \mathrm{H}$ NMR (400 MHz, $\left.\mathrm{CDCl}_{3}\right): \delta 7.35(\mathrm{~m}, 4 \mathrm{H}), 7.13(\mathrm{~d}, 1 \mathrm{H}, J=9.2), 6.87(\mathrm{~d}, 1 \mathrm{H}, J=8.8), 6.22$ (dd, $1 \mathrm{H}, J=6.4,15.6$ ), 6.05 (dd, $1 \mathrm{H}, J=1.6,15.6$ ), 4.57 (dt, $1 \mathrm{H}, J=1.2$, 6.0), 4.51 (m, 1H), 4.4 (t, 1H, $J=4.8), 4.24$ (dd, 1H, $J=5.6,10.0), 3.91$ (dd, 1H, J=4.8, 9.6), 2.31 (s, 3H), 1.31 (s, 9H) ppm; ${ }^{13} \mathrm{C}$ NMR (100 $\left.\mathrm{MHz}_{\mathrm{CDCl}}\right): \delta 154.9,151.6,139.1,1319,131.3,130.3,125.3,119.9$, 115.9, 112.4, 91.1, 86.4, 82.0, 80.6, 72.9, 70.3, 34.8, 31.1, 20.5 ppm; IR (neat): 3347, 2951, 2916, 2854, 1662, 1409, 1266, $1095 \mathrm{~cm}^{-1} ;[\alpha]_{\mathrm{D}}^{23}=-6.0^{\circ}\left(\mathrm{c}=1.0 \mathrm{CH}_{2} \mathrm{Cl}_{2}\right)$.

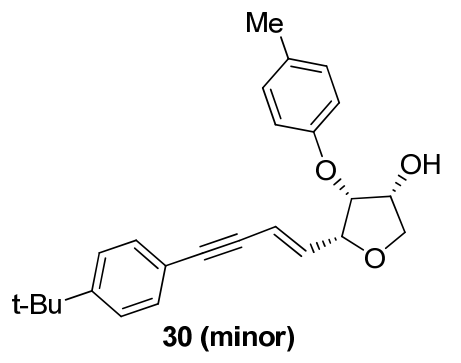

Ene-yne 30 (minor): $6 \mathrm{mg}$ (14\%). Procedure ${ }^{1} \mathrm{H}$ NMR (400 MHz, $\mathrm{CDCl}_{3}$ ): $\delta 7.35$ (m, 4), 7.10(d, $\left.1 \mathrm{H}, J=8.4\right), 6.89$ (d, $\left.1 \mathrm{H}, J=2.4,6.8\right), 6.31$ (dd, $1 \mathrm{H}, J=6.4,16.0$ ), 5.98 (dd, $1 \mathrm{H}, J=1.2,16.0$ ), 4.74 (t, $1 \mathrm{H}, J=5.20$, 4.67 (t, 1H, J=5.6), 4.55 (m, 1H), 4.06 (dd, 1H, J=5.2, 9.6), 3.99 (dd, $1 \mathrm{H}, J=4.4,9.6), 2.23$ (s, 3H), 1.32 (s, 9H) ppm; ${ }^{13} \mathrm{C}$ NMR (100 MHz, $\left.\mathrm{CDCl}_{3}\right): \delta 155.8,151.5,137.9,131.7,131.3,130.2,125.3,120.2,116.0$, 112.9, 90.8, 86.7, 80.1, 79.8, 72.5, 71.7, 34.8, 31.1, 20.5 ppm; IR (neat): 3445, 2955, 1616, 1502, 1460, 1359, 1234, 1091, $955 \mathrm{~cm}^{-1}$; $[\alpha]_{\mathrm{D}}^{23}=-102^{\circ}\left(\mathrm{c}=1.0 \mathrm{CH}_{2} \mathrm{Cl}_{2}\right)$.

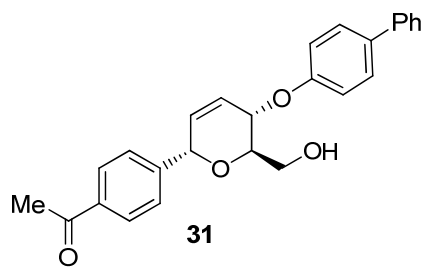

Aryl ether 31: Carbonate 16 was dissolved in $\mathrm{MeOH}(1.5 \mathrm{~mL})$ and MP-CO $\mathrm{CO}_{3}$ (2.98 mmol/g loading, $17 \mathrm{mg}, 0.05 \mathrm{mmol}$ ) was added. After shaking for $15 \mathrm{~h}$, the reaction was concentrated and purified by $\mathrm{SiO}_{2}$ chromatography (0-80\% ethyl acetate / hexanes) to provide 31 (70 mg, $69 \%)$ as a viscous oil. ${ }^{1} \mathrm{H}$ NMR $\left(400 \mathrm{MHz}, \mathrm{CDCl}_{3}\right): \delta 7.99(\mathrm{~d}, 2 \mathrm{H}$, $J=8.4), 7.56$ (m, 6H), 7.42 (t, 2H, $J=7.2), 7.32$ (t, 1H, $J=7.2), 7.03$ (d, 2H, $J=8.8), 6.22$ (m, 2H), 5.42 (s, 1H0, 4.99 (d, 1H, $J=8.4), 3.75$ (m, 3H), 2.62 (s, 3H) ppm; ${ }^{13} \mathrm{C}$ NMR $\left(100 \mathrm{MHz}, \mathrm{CDCl}_{3}\right): \delta 197.7,156.8,144.2,104.6,136.8,134.6,129.3,128.7,128.6,128.4,127.9$, 127.0, 126.8, 126.7, 116.0, 73.8, 71.3, 68.2, 62.1, 26.7 ppm; IR (neat): 3449, 2897, 1678, 1608, 1515, 1487, 1405, 1262, $1087 \mathrm{~cm}^{-1}$; HRMS (Tof) [M+H] $]^{+}$: calcd. for $\mathrm{C}_{26} \mathrm{H}_{24} \mathrm{O}_{4} 401.1753$, found 401.1700. $[\alpha]_{\mathrm{D}}^{23}=+105^{\circ}\left(\mathrm{c}=1.0 \mathrm{CH}_{2} \mathrm{Cl}_{2}\right)$. 


\section{Synthesis of Galactal-Derived tetrahydrofurans 40 and 41:}

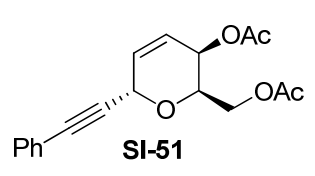

Alkynyl C-Galactoside SI-51: Tri-O-Acetyl-D-Galactal (36) and 1-phenyl-2trimethylsilyl- acetylene were dissolved in $\mathrm{CH}_{2} \mathrm{Cl}_{2}$. The reaction was cooled to $-25^{\circ} \mathrm{C}$ and $\mathrm{Sc}(\mathrm{OTf})_{3}$ was added. After $3.5 \mathrm{~h}$, the reaction was quenched with saturated sodium bicarbonate (at $-25^{\circ} \mathrm{C}$ ), diluted with $\mathrm{CH}_{2} \mathrm{Cl}_{2}$, then washed with sodium bicarbonate $(1 \mathrm{X})$ and brine $(1 \mathrm{X})$. The organic layer was dried, filtered, concentrated onto silica, and purified over $\mathrm{SiO}_{2}$ (0-40\% ethyl acetate/petroleum ether) yielding $\mathbf{S I - 5 1}$ as a white solid. ${ }^{1} \mathrm{H}$ NMR (400 MHz; $\left.\mathrm{CDCl}_{3}\right): \delta 7.44(\mathrm{~m}, 2 \mathrm{H}), 7.33(\mathrm{~m}, 3 \mathrm{H}), 6.15$ (dd, $\left.1 \mathrm{H}, J=3.6,10.4\right), 6.05$ (ddd, $1 \mathrm{H}, J=2.0,4.8,6.8$ ), 5.27 (dd, $1 \mathrm{H}, J=2.0,4.0$ ), 5.12 (dd, $1 \mathrm{H}, J=2.4,5.2$ ), 4.45 (td, $1 \mathrm{H}, J=2.0,4.8$, 7.2), 4.32 (dd, $1 \mathrm{H}, J=5.6,11.6$ ), 4.24 (dd, $1 \mathrm{H}, J=7.2,11.2$ ), 2.10 (s, 3H) , 2.10 (s, 3H) ppm ; ${ }^{13} \mathrm{C}$ NMR (100MHz, $\left.\mathrm{CDCl}_{3}\right): \quad \delta 171.7,170.4,132.0,131.8,128.8,128.3,122.4,122.1,86.9,84.1,69.7$, 64.4, 64.4, 63.3, 62.8, 20.9, 20.8 ppm; IR (neat): 3739, 1744, 1367, 1231, 1076, 1045, 761 $\mathrm{cm}^{-1}$; HRMS (Tof) $[\mathrm{M}+\mathrm{Na}]^{+}$: calcd. for $\mathrm{C}_{18} \mathrm{H}_{18} \mathrm{O}_{5} \mathrm{Na} 337.1052$, found 337.0977. $[\alpha]_{\mathrm{D}}{ }^{23}=-396.9^{\circ}(\mathrm{c}=1.0$ $\mathrm{CH}_{2} \mathrm{Cl}_{2}$ ).

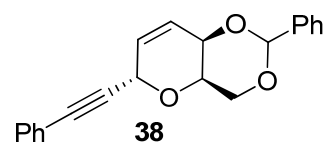

Acetonide 38: Diacetate SI-51 (600 mg, $1.91 \mathrm{mmol}$ ) was dissolved in $\mathrm{MeOH}$ (8 $\mathrm{mL}$ ). $\mathrm{K}_{2} \mathrm{CO}_{3}(132 \mathrm{mg}, 9.54 \mathrm{mmol})$ was added, the reaction was stirred for 40 min at room temperature and was concentrated onto silica and purified over $\mathrm{SiO}_{2}$ (0-100\% ethyl acetate/ petroleum ether) to provide the intermediate diol 37 (409 mg, $1.78 \mathrm{mmol}$, 93\%) as white flakes. ${ }^{1} \mathrm{H}$ NMR (400 MHz; $\left.\mathrm{CDCl}_{3}\right): \delta 7.42(\mathrm{~m}, 2 \mathrm{H}), 7.29(\mathrm{~m}, 3 \mathrm{H}), 6.09(\mathrm{~m}, 1 \mathrm{H})$, 6.04 (dd, $1 \mathrm{H}, J=2.0,5.6), 5.23$ (dd, $1 \mathrm{H}, J=2.0,3.6), 4.15$ (m 1H), 3.97 (m, $1 \mathrm{H}), 3.97(\mathrm{~m}, 1 \mathrm{H})$, 2.51(bs, $1 \mathrm{H}), 2.36$ (bs, $1 \mathrm{H}) \mathrm{ppm} ;{ }^{13} \mathrm{C}$ NMR $\left(100 \mathrm{MHz}, \mathrm{CDCl}_{3}\right): \delta 131.8,130.0,128.7,128.3,126.6$, 122.1, 86.6, 84.6, 73.4,, 64.5, 62.8 ppm; IR (neat): 3305, 1604, 1317, 1115, 1080, $765 \mathrm{~cm}^{-1}$; $[\alpha]_{\mathrm{D}}^{23}=-52.6^{\circ}\left(\mathrm{c}=1.0 \mathrm{CH}_{2} \mathrm{Cl}_{2}\right)$.

Diol 37 (100 mg, $0.43 \mathrm{mmol}$ ) was dissolved in $\mathrm{CH}_{2} \mathrm{Cl}_{2}(4 \mathrm{~mL})$. 10-Camphorsulfonic acid (7 mg, $0.03 \mathrm{mmol})$ and benzaldehyde dimethyl acetal $(0.11 \mathrm{~mL}, 0.75 \mathrm{mmol})$ were added, the reaction was stirred for $12 \mathrm{~h}$ then diluted with $\mathrm{CH}_{2} \mathrm{Cl}_{2}$, washed with brine (1X), and back-extracted with $\mathrm{CH}_{2} \mathrm{Cl}_{2}$. The organic layers were combined, dried, filtered, concentrated onto $\mathrm{SiO}_{2}$, and purified over $\mathrm{SiO}_{2}$ (0-40\% ethyl acetate/petroleum ether) to provide 38 (68 mg, $0.21 \mathrm{mmol}, 49 \%)$ as a white solid. ${ }^{1} \mathrm{H}$ NMR (400MHz; $\left.\mathrm{CDCl}_{3}\right): \delta 7.55$ (m, 2H), 7.45 (m,2H), 7.34 (m, 6H), 6.17 (dd, $\left.1 \mathrm{H}, J=3.6,10.4\right)$, 6.06 (ddd, $1 \mathrm{H}, J=2.0,5.6,10.0), 5.62$ (s, $1 \mathrm{H}$ ), 5.38 (m, 1H), 4.45 (d, $1 \mathrm{H}, J=12.8$ ), 4.28 (dd, $1 \mathrm{H}, J=$ 2.8, 17.2), 4.27 (s, $1 \mathrm{H}), 3.97$ (bs, $1 \mathrm{H}) \mathrm{ppm} ;{ }^{13} \mathrm{C} \mathrm{NMR}\left(100 \mathrm{MHz}, \mathrm{CDCl}_{3}\right): \delta 137.8,131.8,128.8$, 128.7, 128.3, 128.1, 126.2, 123.2, 122.2, 100.7, 86.5, 85.1, 71.2, 67.9, 64.6 ppm; IR (neat): 3033, $2909,1689,1592,1328,750,694 \mathrm{~cm}^{-1} ; \quad[\alpha]_{\mathrm{D}}^{23}=-365.8^{\circ}\left(\mathrm{c}=1.0 \mathrm{CH}_{2} \mathrm{Cl}_{2}\right)$.

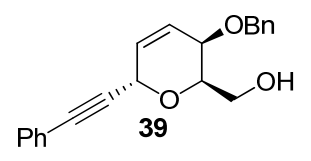

Alcohol 39: Acetonide 38 (70 mg, $0.22 \mathrm{mmol})$ was dissolved in $\mathrm{CH}_{2} \mathrm{Cl}_{2}(2 \mathrm{~mL})$ 
and cooled to $0^{\circ} \mathrm{C}$. A $1 \mathrm{M}$ solution of Diisobutylaluminum hydride in hexanes $(1.76 \mathrm{~mL}, 1.76 \mathrm{mmol})$ was added and stirred for 2 hours. The reaction was quenched with saturated ammonium chloride and saturated sodium tartrate, stirred for one hour, then diluted with $\mathrm{CH}_{2} \mathrm{Cl}_{2}$ and washed with sodium bicarbonate. The organic layer was dried, filtered, concentrated onto silica, and purified over $\mathrm{SiO}_{2}$ (0-40\% ethyl acetate/petroleum ether) to provide 39 (35 mg, $0.11 \mathrm{mmol}$ ) as a white solid. ${ }^{1} \mathrm{H}$ NMR (400MHz; $\left.\mathrm{CDCl}_{3}\right): \delta 7.43(\mathrm{~m}, 2 \mathrm{H}), 7.32(\mathrm{~m}, 8 \mathrm{H}), 6.14$ (m, 2H), 5.30 (d, 1H, J = 1.2), 4.71 (d, $1 \mathrm{H}, J$ = 11.6), $4.56(\mathrm{~d}, 1 \mathrm{H}, J=12.0), 4.19(\mathrm{~m}, 1 \mathrm{H}), 4.02(\mathrm{~m}, 1 \mathrm{H}), 3.85(\mathrm{~m}, 1 \mathrm{H}), 2.20(\mathrm{bs}, 1 \mathrm{H}) \mathrm{ppm} ;{ }^{13} \mathrm{C}$ NMR (100MHz, $\left.\mathrm{CDCl}_{3}\right): \delta 138.0,131.8,131.3,128.6,128.5,128.3,128.0,127.8,123.6,122.2,86.5$, 84.9, 73.5, 70.7, 68.2, 64.4, 62.7 ppm; IR (neat): 3939, 3052, 2916, 1631, 1588, 1324, 1087, 757, 699 $\mathrm{cm}^{-1} ;[\alpha]_{\mathrm{D}}^{23}=-265.5^{\circ}\left(\mathrm{c}=1.0 \mathrm{CH}_{2} \mathrm{Cl}_{2}\right)$.

Furan 40,41: Alcohol 39 (72 mg, 0.22mmol) and gold (III) chloride (27 mg, $0.09 \mathrm{mmol}$ ) were added to a round bottom flask, and dissolved in $\mathrm{CH}_{2} \mathrm{Cl}_{2}(1 \mathrm{~mL})$. After $4 \mathrm{~h}$, the reaction was concentrated onto silica gel and purified over $\mathrm{SiO}_{2}$ (0-40\% ethyl acetate/ petroleum ether) to provide $\mathbf{4 0}$ (26.4 mg, $37 \%)$ as an oil and $\mathbf{4 1}$ (10.6 mg, 15\%) as a film.

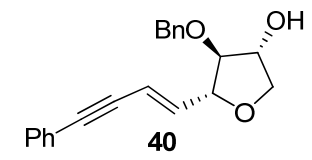

Compound 40: ${ }^{1} \mathrm{H}$ NMR (400MHz; $\left.\mathrm{CDCl}_{3}\right): \delta 7.40$ (m, 2H), 7.08 (m, 3H), 6.93 (m, 3H), 6.37 (dd, 1H, J=6.0, 15.6), 6.08 (dd, 1H, $J=1.6,15.6), 4.33$ (m, 1H), 4.21 (s, 2H), 3.87 (bs, 1H), 3.75 (dd, 1H, $J=4.4,10.0$ ), 3.58 (dd, 1H, $J=2.0$, 10.0), 3.54 (dd, $1 \mathrm{H}, J=1.6,3.6) \mathrm{ppm} ;{ }^{13} \mathrm{C} \mathrm{NMR}\left(400 \mathrm{M} ; \mathrm{CDCl}_{3}\right): \delta 140.9,137.5,131.5,128.6$, 128.3, 128.0, 127.7, 123.1, 111.2, 90.7, 89.6, 87.1, 83.9, 76.4, 74.2, 72.2 ppm; IR (neat): 3424, 3032, 3870, 1094, 959, 753, $694 \mathrm{~cm}^{-1} ; \quad[\alpha]_{\mathrm{D}}^{23}=+80.0^{\circ}(\mathrm{c}=13.4 \mathrm{mg} / \mathrm{mL})$

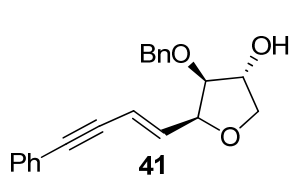

Compound 41: ${ }^{1} \mathrm{H}$ NMR (400MHz; $\left.\mathrm{CDCl}_{3}\right): \delta 7.37$ (m, 2H), 7.19 (m, 2H), 7.09 (m, 2H), 6.90 (m, 3H), 6.56 (dd, $1 \mathrm{H}, J=6.8,16.0), 6.13$ (dd, $1 \mathrm{H}, J=1.6,13.2$ ), 4.46, (m, 1H), 4.20 (s, 2H), 3.96 (dd, 1H, J=4.4, 9.2), 3.84 (m, 1H), 3.46 (dd, $1 \mathrm{H}, J=1.6,4.0), 3.43$ (dd, $1 \mathrm{H}, J=2.0,9.2) \mathrm{ppm} ;{ }^{13} \mathrm{C} \mathrm{NMR}\left(400 \mathrm{M} ; \mathrm{CDCl}_{3}\right)$ : 8138.3, 137.7, 131.6, 128.5, 128.3, 128.2, 127.9, 127.7, 123.3, 112.7, 90.3, 87.5, 85.7, 80.7, 75.7, 73.6, $72.5 \mathrm{ppm}$; IR (neat): 3408, 3032, 2925, 1091, 962, 755, 693 $\mathrm{cm}^{-1} ;[\alpha]_{\mathrm{D}}^{23}=-18.8^{0}(\mathrm{c}=1.0$ $\left.\mathrm{CH}_{2} \mathrm{Cl}_{2}\right)$ 


\section{Examples of Selected Spectral Data}
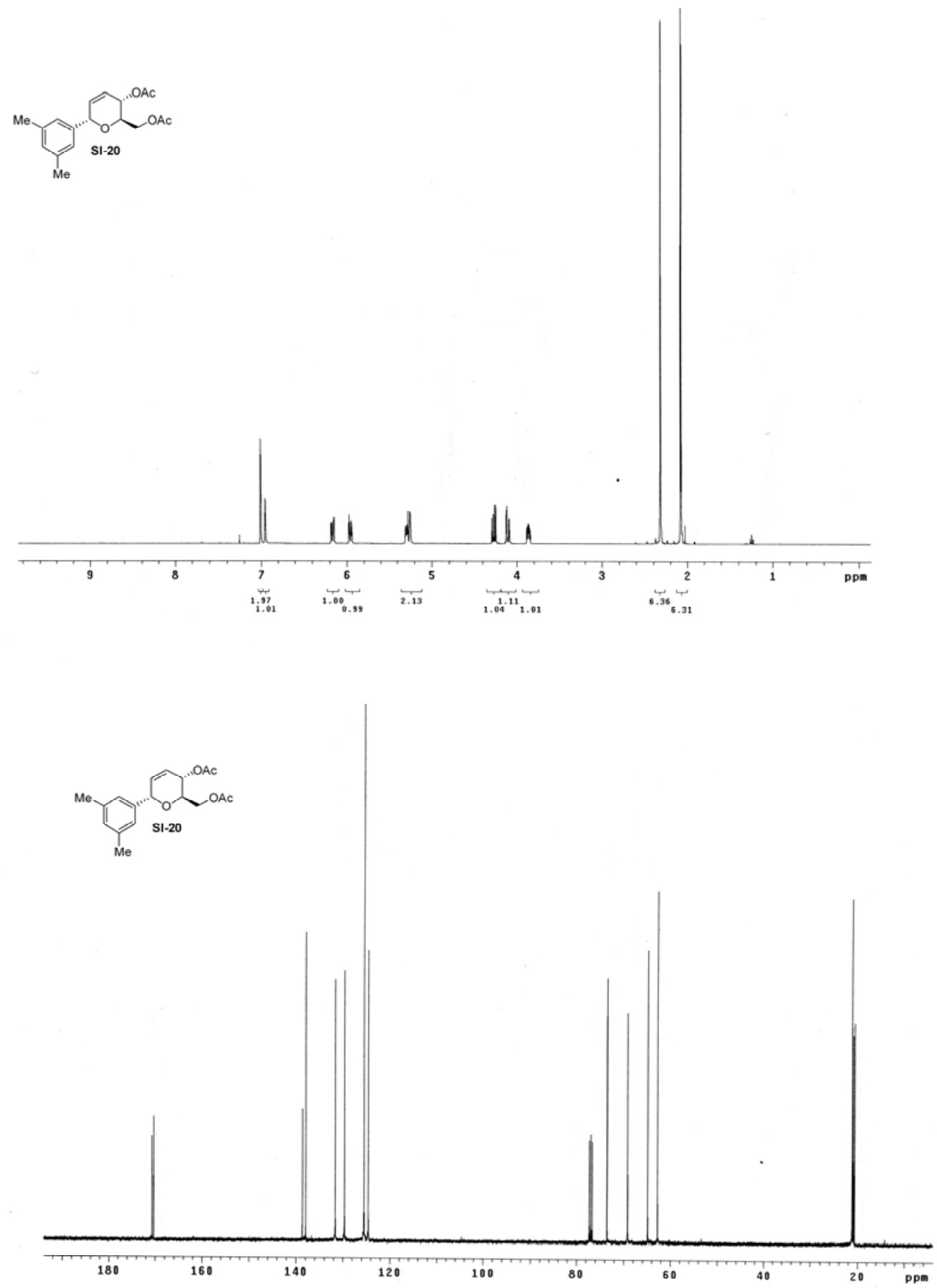


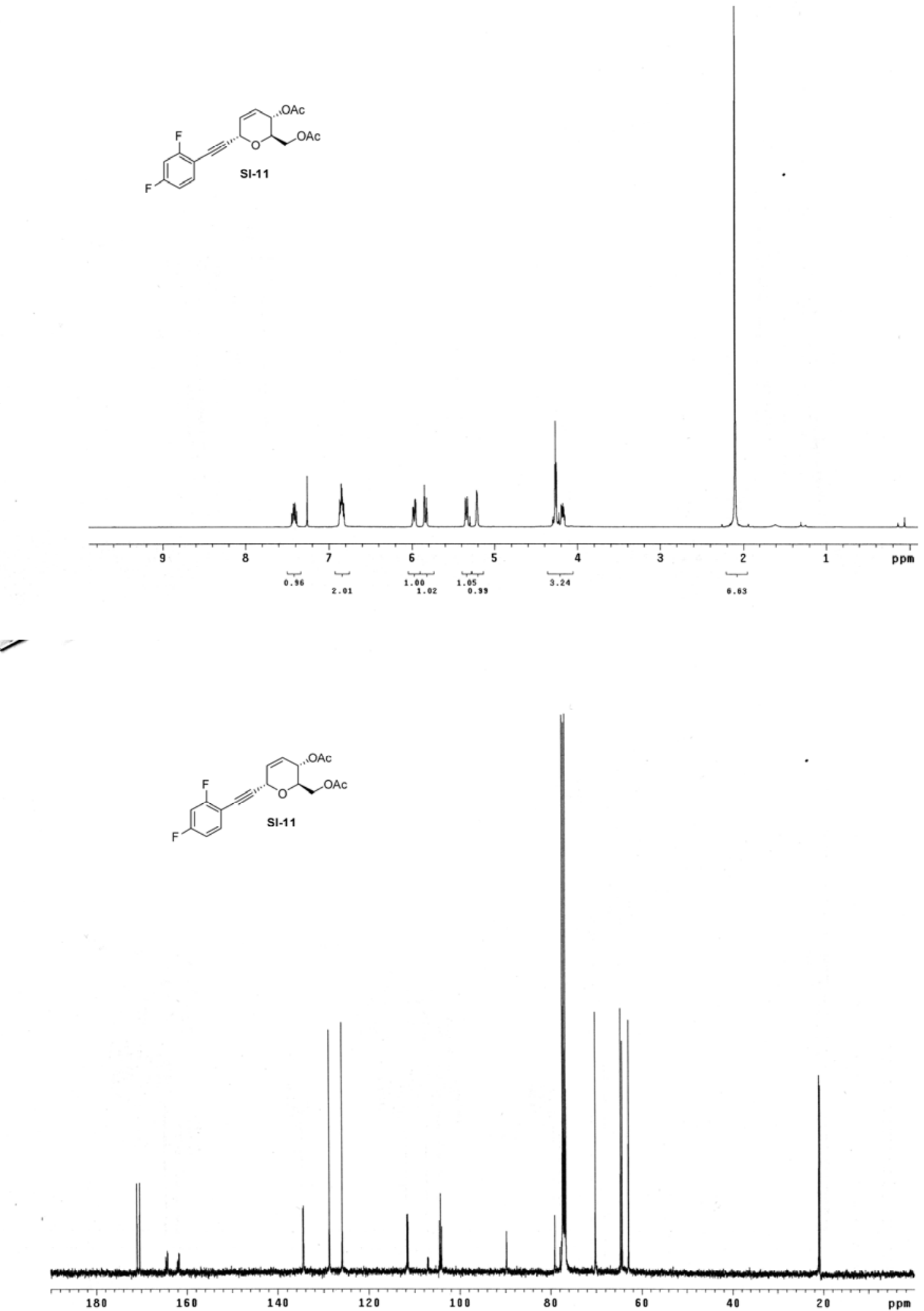



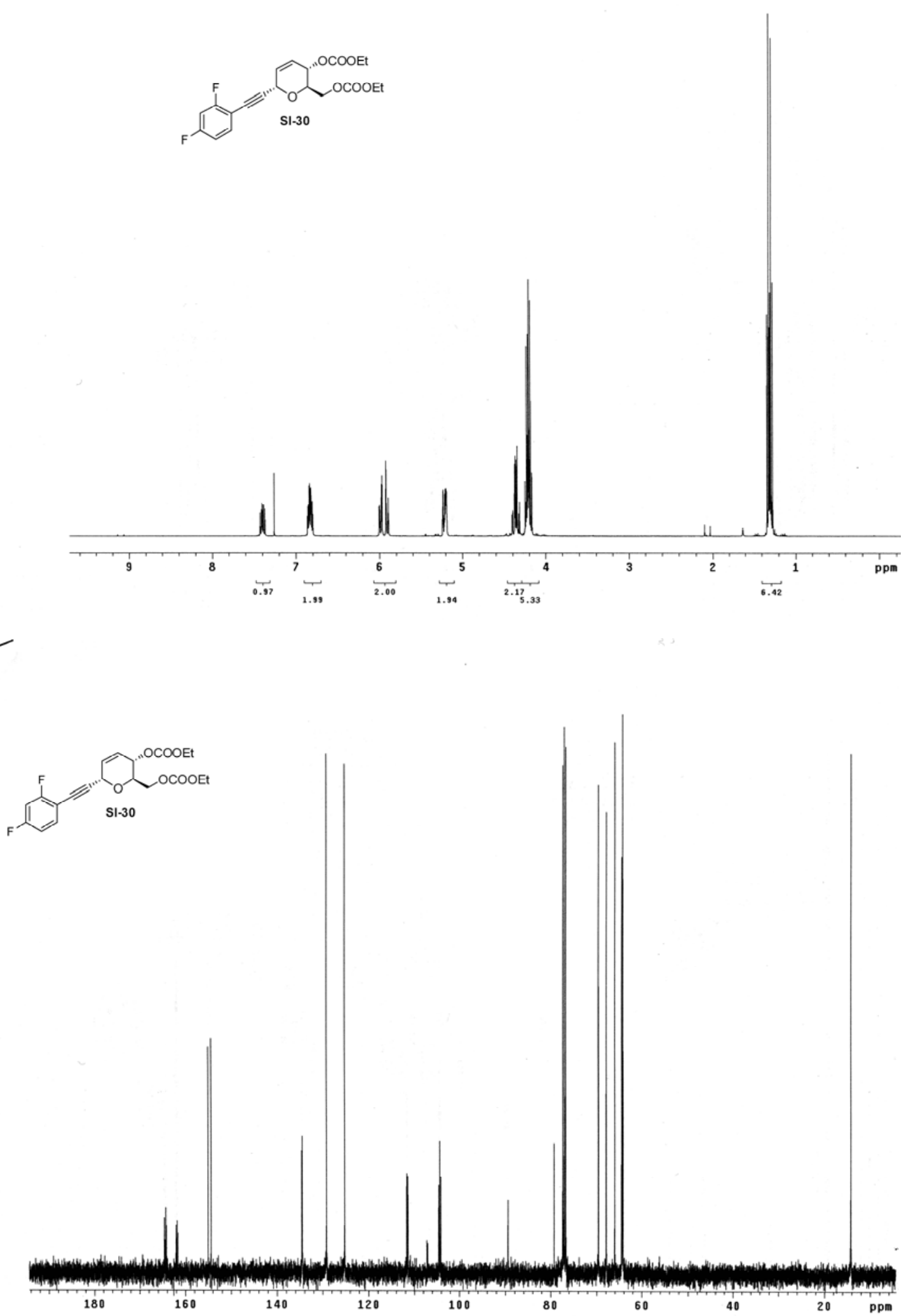

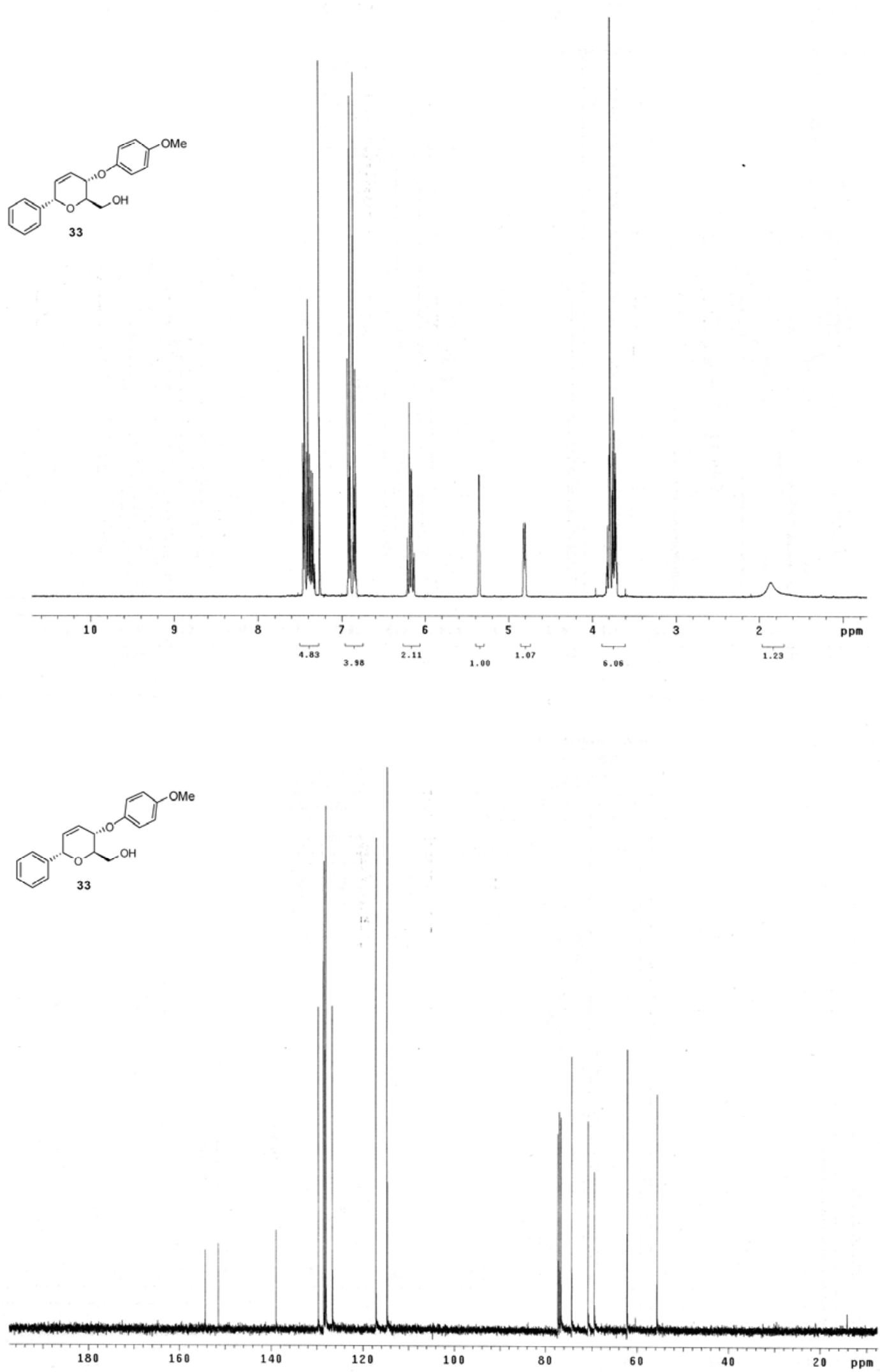

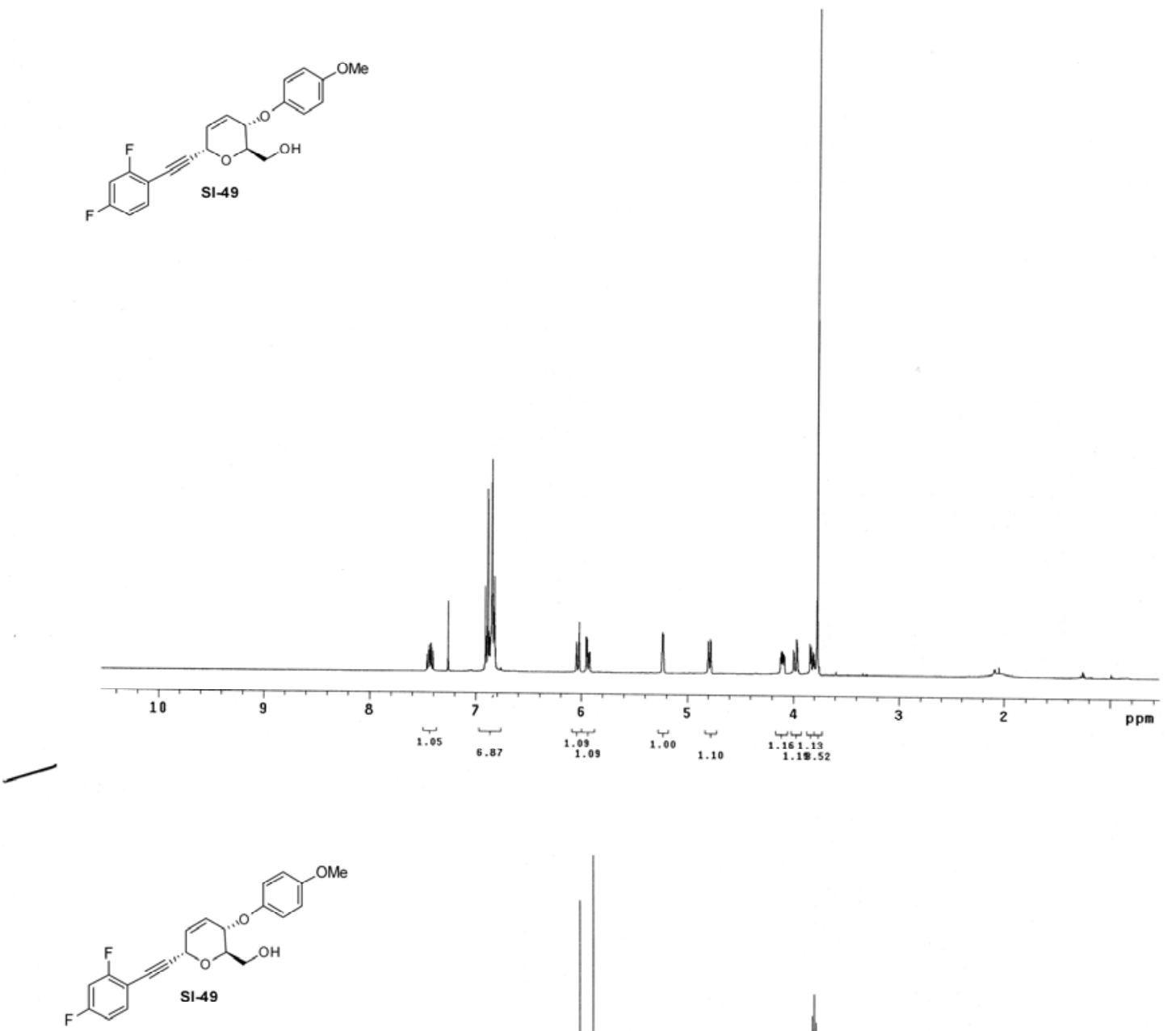

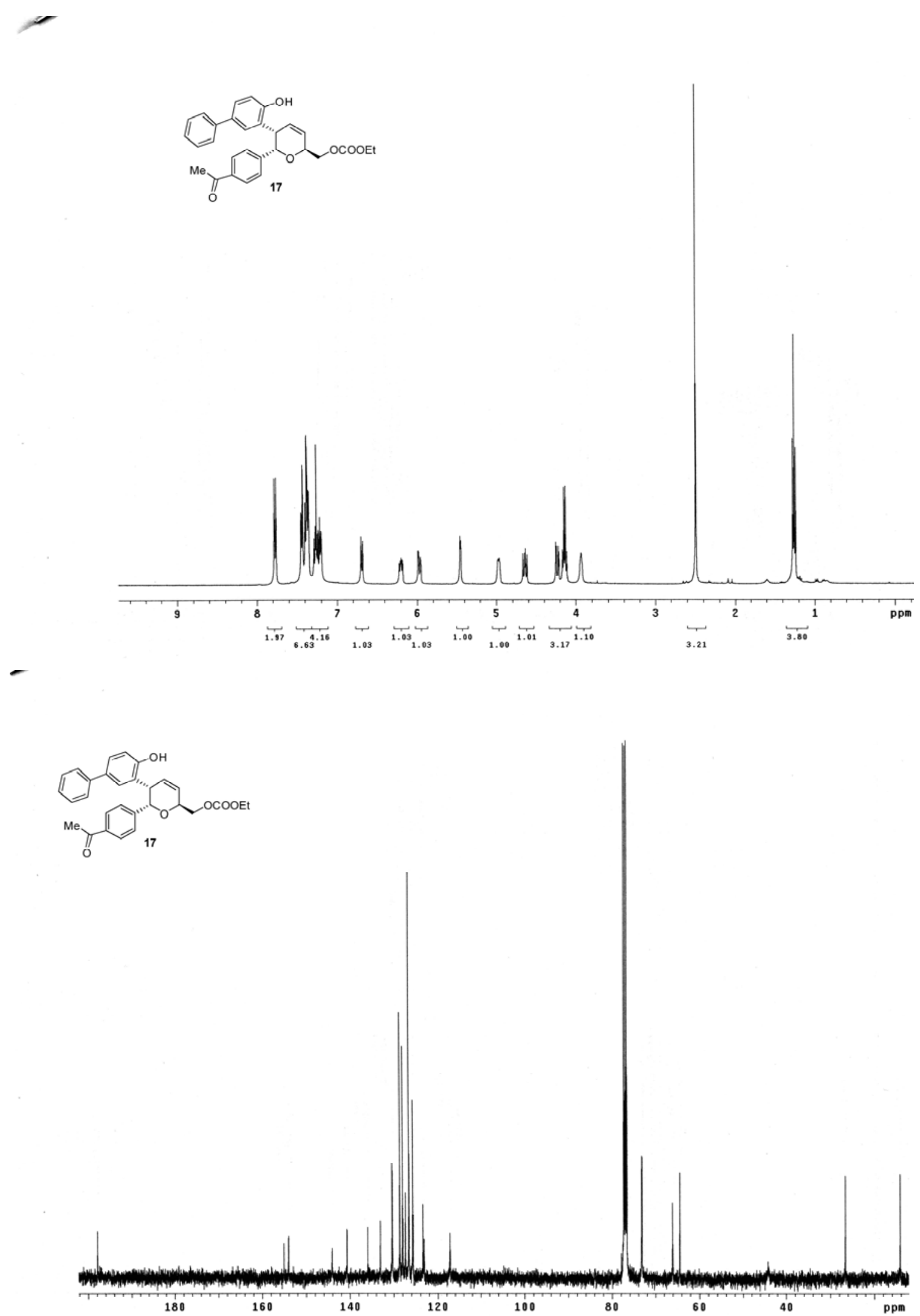

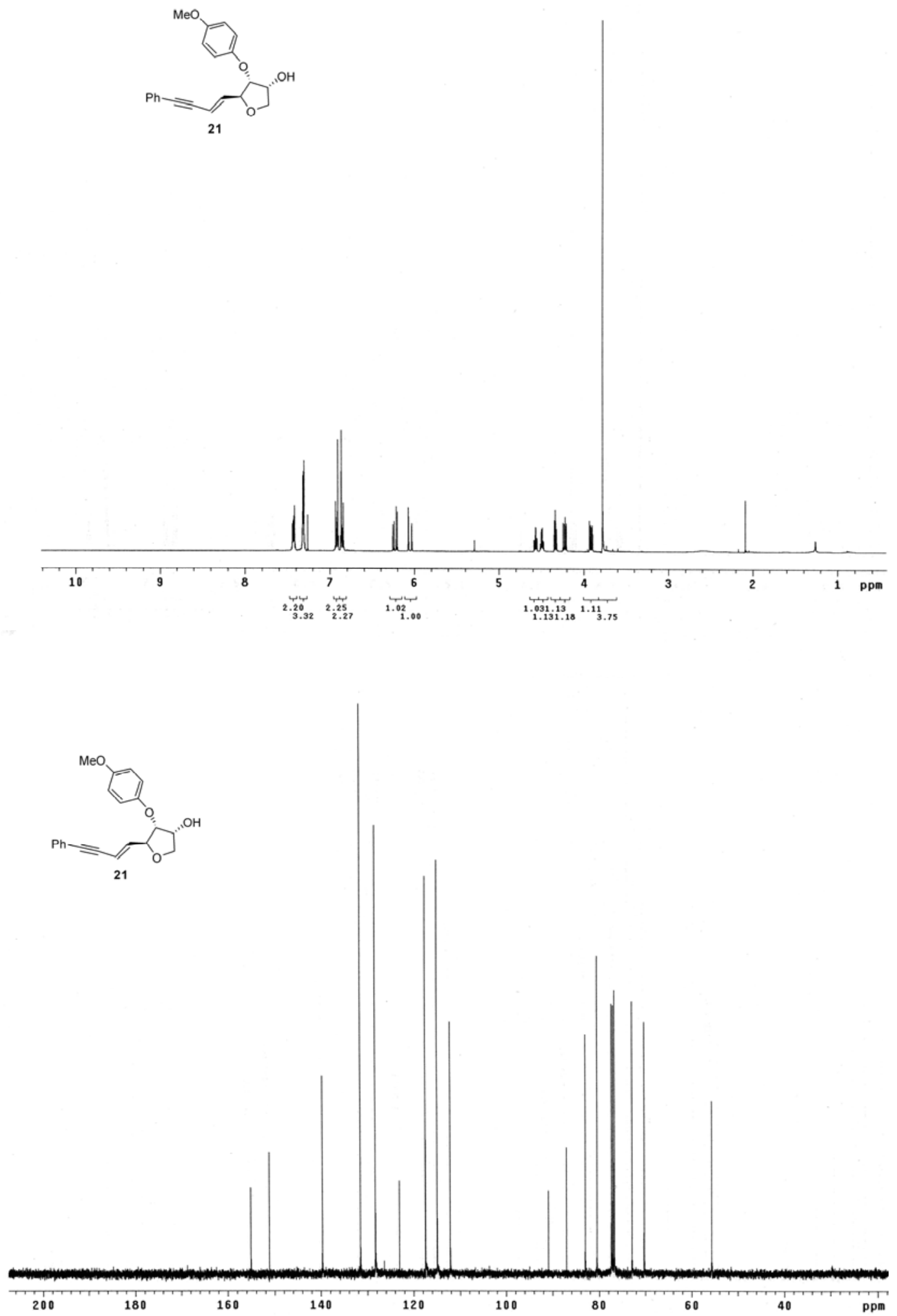

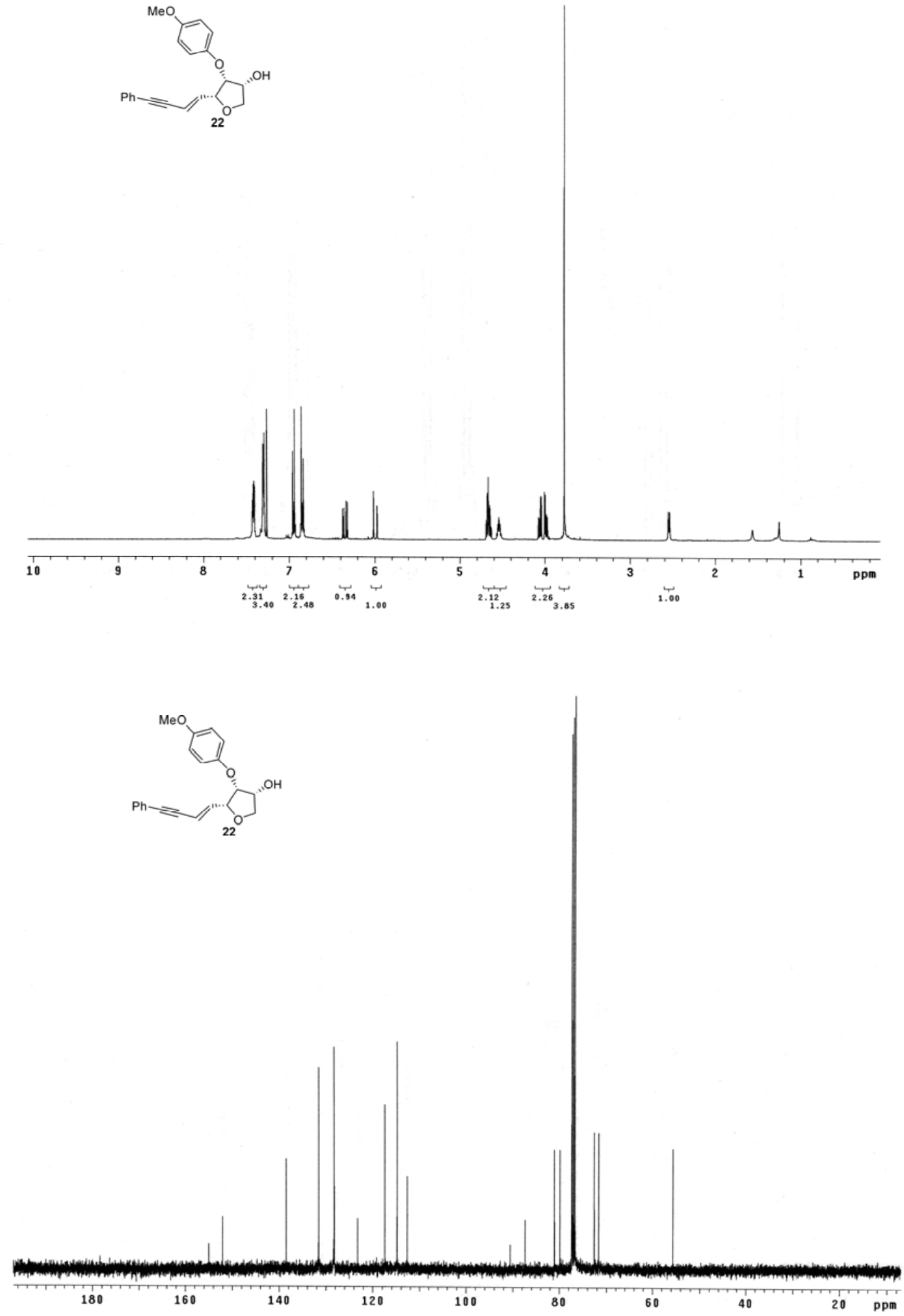
Figure 1. Time dependent ${ }^{1} \mathrm{H}$ NMR experiments of $\mathrm{AuCl}_{3}$-mediated formation of tetrahydrofurans 24 and $25 .{ }^{1} \mathrm{H}$ NMR's taken in $\mathrm{C}_{6} \mathrm{D}_{6}$ of crude $\mathrm{SiO}_{2}$-filtered reaction mixtures.
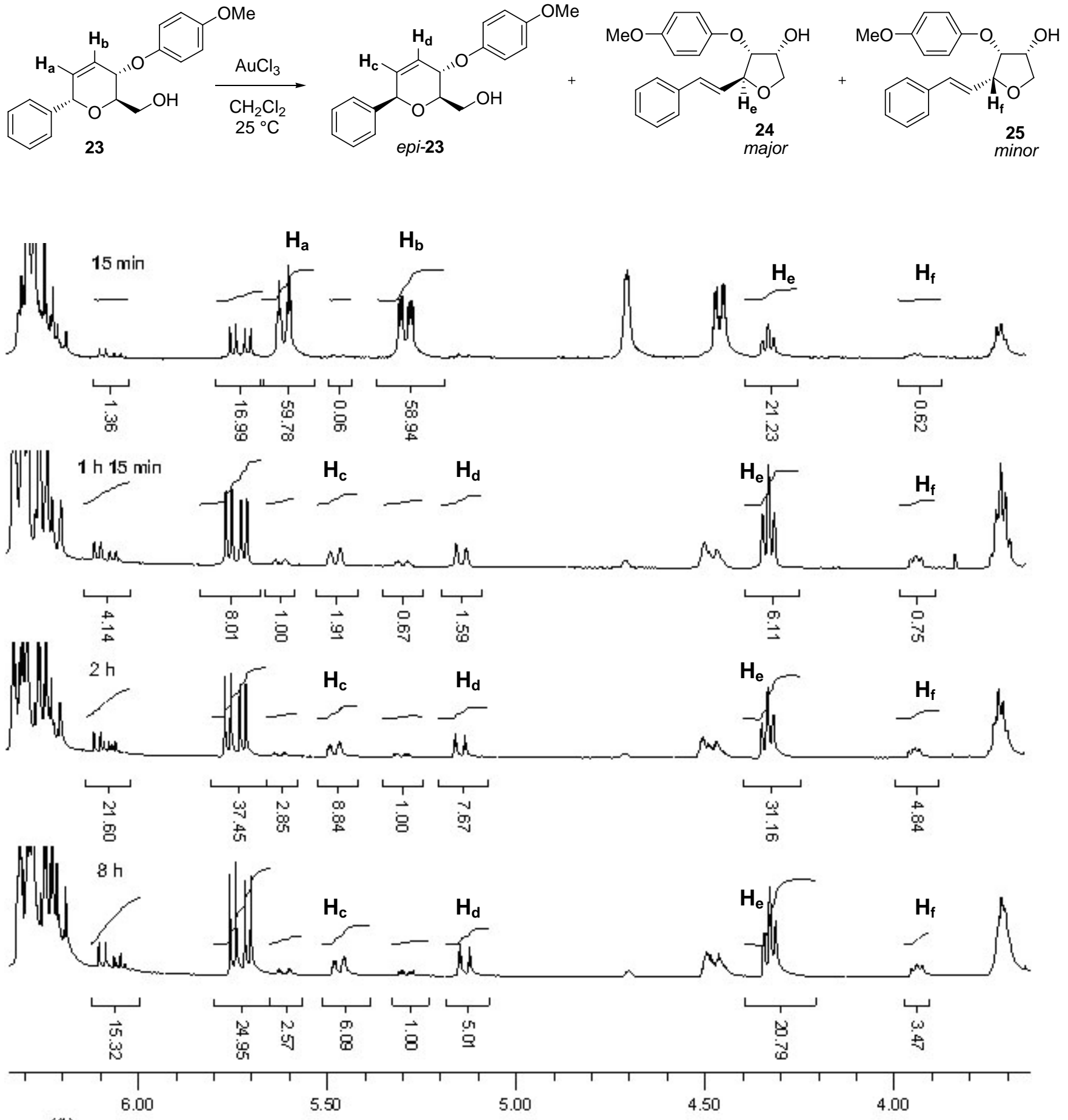

porn (f1) 
Figure 2. ${ }^{1} \mathrm{H}$ NMR experiments of epimeric 23 produced during the ring contraction reaction. ${ }^{1} \mathrm{H}$ NMR taken in $\mathrm{C}_{6} \mathrm{D}_{6}$.
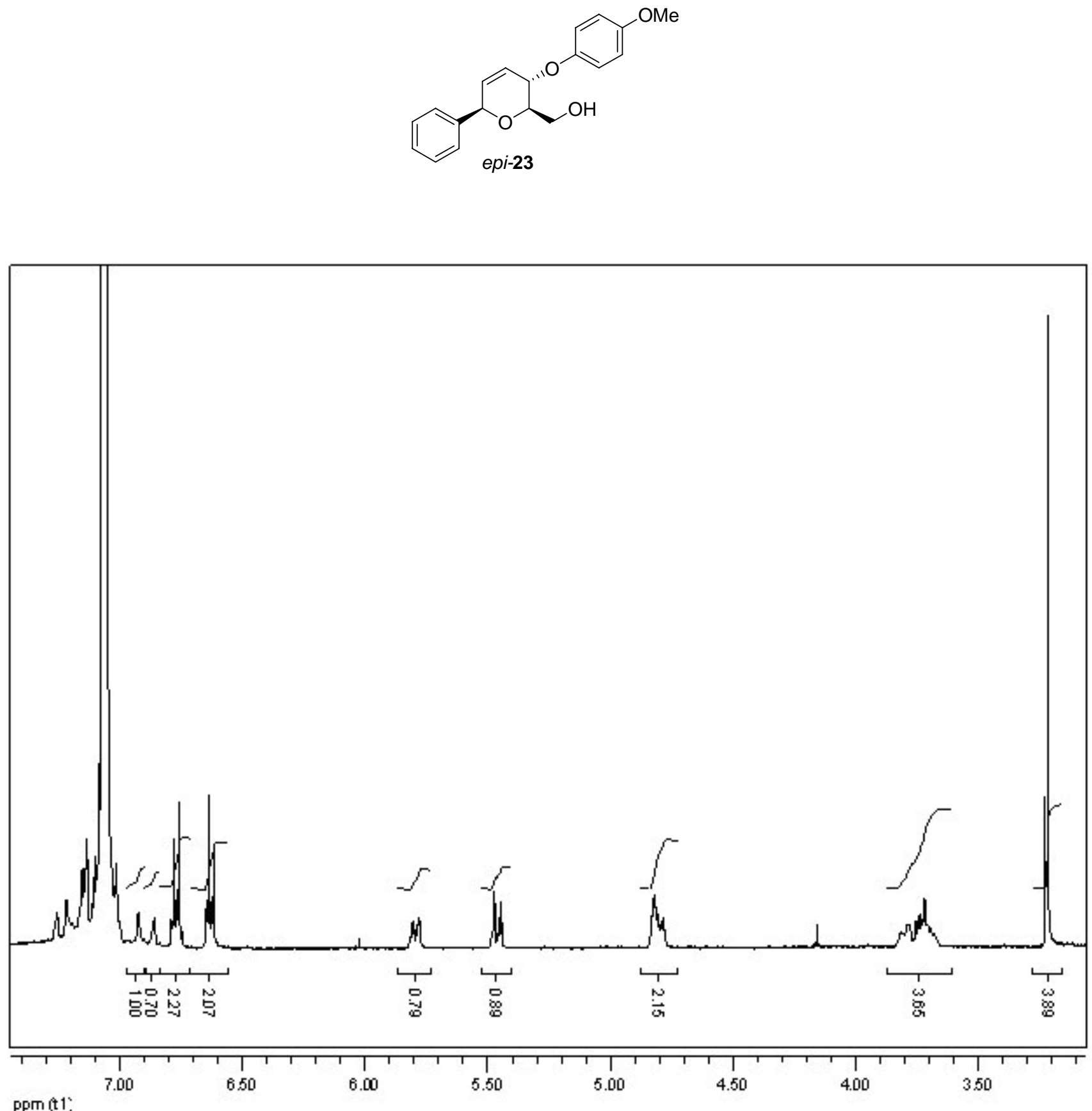


\section{X-ray Crystal Structure of Compound SI-15:}

Crystals of compound SI-15 suitable for x-ray analysis were obtained by slow evaporation from hexanes. Crystallographic data have been deposited with the Cambridge Crystallographic Data Centre (CCDC \# 260139). Copies of the data can be obtained free of charge on application to the CCDC, 12 Union Road, Cambridge CB21EZ, UK (fax: (+44)-1223-336-033; e-mail: deposit@ccdc.cam.ac.uk.

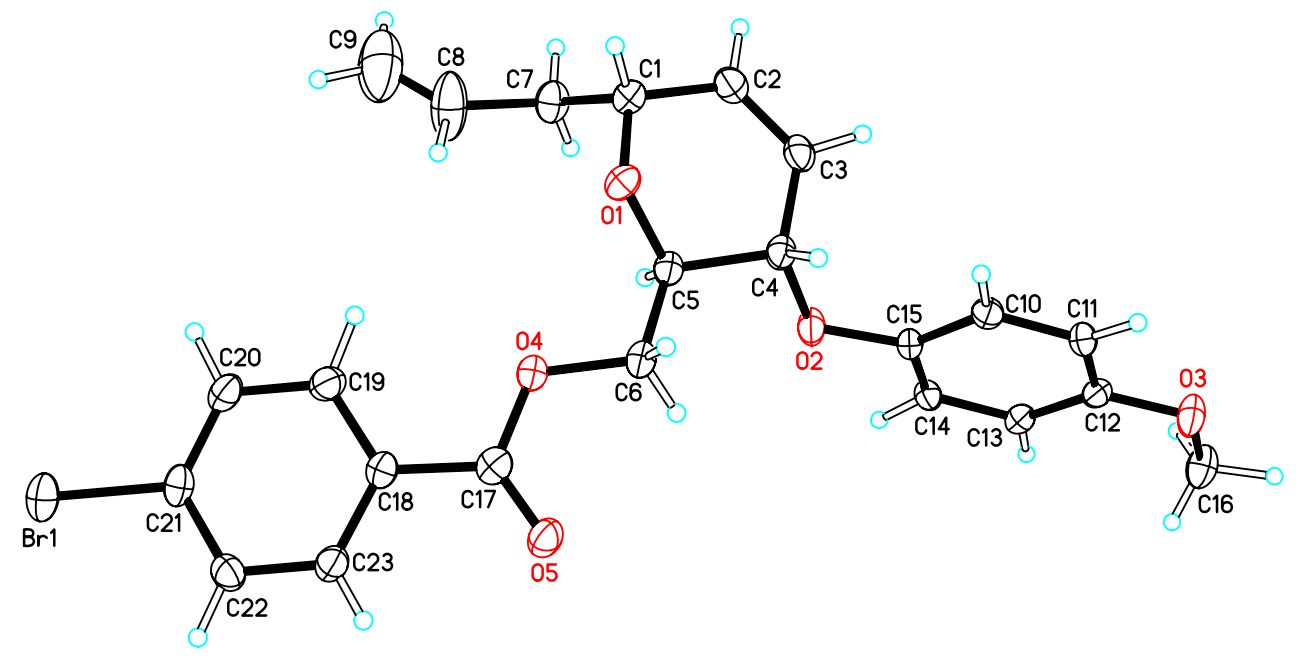

Table 1. Crystal data and structure refinement for porco29.

Identification code

Empirical formula

Formula weight

Temperature

Wavelength

Crystal system

Space group

Unit cell dimensions

Volume

Z

Density (calculated)

Absorption coefficient

$\mathrm{F}(000)$ porco29

C23 H23 Br O5

459.32

173(2) K

$0.71073 \AA$

Monoclinic

P2(1)

$$
\begin{array}{ll}
\mathrm{a}=10.0462(7) \AA & \alpha=90^{\circ} . \\
\mathrm{b}=5.3870(4) \AA & \beta=100.504(2)^{\circ} . \\
\mathrm{c}=19.6191(14) \AA & \gamma=90^{\circ} .
\end{array}
$$

1043.97(13) $\AA^{3}$

2

$1.461 \mathrm{Mg} / \mathrm{m}^{3}$

$2.000 \mathrm{~mm}^{-1}$

472 
Crystal size

Theta range for data collection

Index ranges

Reflections collected

Independent reflections

Completeness to theta $=30.51^{\circ}$

Absorption correction

Max. and min. transmission

Refinement method

Data / restraints / parameters

Goodness-of-fit on $\mathrm{F}^{2}$

Final R indices [I>2sigma(I)]

$\mathrm{R}$ indices (all data)

Absolute structure parameter

Largest diff. peak and hole
$1.00 \times 0.30 \times 0.15 \mathrm{~mm}^{3}$

2.48 to $30.51^{\circ}$.

$-14<=\mathrm{h}<=14,-6<=\mathrm{k}<=7,-24<=\mathrm{l}<=28$

8041

$5322[\mathrm{R}(\mathrm{int})=0.0252]$

$97.8 \%$

\section{SADABS}

0.7535 and 0.2396

Full-matrix least-squares on $\mathrm{F}^{2}$

5322 / 1 / 343

0.964

$\mathrm{R} 1=0.0384, \mathrm{wR} 2=0.0819$

$\mathrm{R} 1=0.0539, \mathrm{wR} 2=0.0869$

0.055(10)

0.567 and -0.503 e. $\AA^{-3}$

Table 2. Atomic coordinates ( $\mathrm{x} 10^{4}$ ) and equivalent isotropic displacement parameters $\left(\AA^{2} \times 10^{3}\right)$ for porco29. $\mathrm{U}(\mathrm{eq})$ is defined as one third of the trace of the orthogonalized $\mathrm{U}^{\mathrm{ij}}$ tensor.

\begin{tabular}{lrrrr}
\hline & $\mathrm{x}$ & $\mathrm{y}$ & $\mathrm{z}$ & $\mathrm{U}(\mathrm{eq})$ \\
\hline $\mathrm{Br}(1)$ & $944(1)$ & $2677(1)$ & $4190(1)$ & $41(1)$ \\
$\mathrm{O}(1)$ & $-5019(2)$ & $14447(4)$ & $2779(1)$ & $27(1)$ \\
$\mathrm{O}(2)$ & $-8105(2)$ & $11847(3)$ & $1833(1)$ & $30(1)$ \\
$\mathrm{O}(3)$ & $-12856(2)$ & $12867(6)$ & $-91(1)$ & $36(1)$ \\
$\mathrm{O}(4)$ & $-4033(2)$ & $9901(4)$ & $2407(1)$ & $30(1)$ \\
$\mathrm{O}(5)$ & $-3783(2)$ & $7402(5)$ & $1524(1)$ & $33(1)$ \\
$\mathrm{C}(1)$ & $-5547(3)$ & $15648(6)$ & $3321(1)$ & $29(1)$ \\
$\mathrm{C}(2)$ & $-6994(3)$ & $16409(6)$ & $3081(2)$ & $31(1)$ \\
$\mathrm{C}(3)$ & $-7704(3)$ & $15654(5)$ & $2481(2)$ & $29(1)$ \\
$\mathrm{C}(4)$ & $-7166(3)$ & $13852(5)$ & $2019(1)$ & $26(1)$ \\
$\mathrm{C}(5)$ & $-5894(2)$ & $12602(7)$ & $2422(1)$ & $25(1)$ \\
$\mathrm{C}(6)$ & $-5117(3)$ & $11212(6)$ & $1960(2)$ & $30(1)$ \\
$\mathrm{C}(7)$ & $-5341(3)$ & $14054(7)$ & $3983(2)$ & $34(1)$ \\
$\mathrm{C}(8)$ & $-3884(3)$ & $13459(10)$ & $4251(2)$ & $64(2)$ \\
$\mathrm{C}(9)$ & $-3230(5)$ & $13519(10)$ & $4787(3)$ & $89(2)$ \\
& & & &
\end{tabular}




\begin{tabular}{lrrrr}
$\mathrm{C}(10)$ & $-9526(3)$ & $14298(5)$ & $912(1)$ & $26(1)$ \\
$\mathrm{C}(11)$ & $-10738(3)$ & $14430(6)$ & $440(1)$ & $27(1)$ \\
$\mathrm{C}(12)$ & $-11707(2)$ & $12573(8)$ & $407(1)$ & $25(1)$ \\
$\mathrm{C}(13)$ & $-11482(3)$ & $10585(5)$ & $859(1)$ & $25(1)$ \\
$\mathrm{C}(14)$ & $-10262(3)$ & $10435(5)$ & $1333(1)$ & $24(1)$ \\
$\mathrm{C}(15)$ & $-9288(2)$ & $12262(5)$ & $1353(1)$ & $23(1)$ \\
$\mathrm{C}(16)$ & $-13857(3)$ & $10966(8)$ & $-119(2)$ & $41(1)$ \\
$\mathrm{C}(17)$ & $-3451(2)$ & $8036(7)$ & $2117(1)$ & $27(1)$ \\
$\mathrm{C}(18)$ & $-2340(2)$ & $6859(5)$ & $2619(1)$ & $24(1)$ \\
$\mathrm{C}(19)$ & $-1939(2)$ & $7774(9)$ & $3294(1)$ & $31(1)$ \\
$\mathrm{C}(20)$ & $-944(3)$ & $6523(6)$ & $3761(1)$ & $32(1)$ \\
$\mathrm{C}(21)$ & $-371(2)$ & $4424(6)$ & $3545(1)$ & $28(1)$ \\
$\mathrm{C}(22)$ & $-721(3)$ & $3513(6)$ & $2878(2)$ & $31(1)$ \\
$\mathrm{C}(23)$ & $-1715(3)$ & $4776(6)$ & $2413(1)$ & $29(1)$ \\
& & & & \\
\hline
\end{tabular}


Table 3. Bond lengths $[\AA]$ and angles [ $\left[^{\circ}\right]$ for porco29.

\begin{tabular}{|c|c|}
\hline $\operatorname{Br}(1)-C(21)$ & $1.903(3)$ \\
\hline $\mathrm{O}(1)-\mathrm{C}(5)$ & $1.423(4)$ \\
\hline $\mathrm{O}(1)-\mathrm{C}(1)$ & $1.427(3)$ \\
\hline $\mathrm{O}(2)-\mathrm{C}(15)$ & 1.393(3) \\
\hline $\mathrm{O}(2)-\mathrm{C}(4)$ & $1.437(3)$ \\
\hline $\mathrm{O}(3)-\mathrm{C}(12)$ & $1.379(3)$ \\
\hline $\mathrm{O}(3)-\mathrm{C}(16)$ & $1.429(4)$ \\
\hline $\mathrm{O}(4)-\mathrm{C}(17)$ & $1.341(4)$ \\
\hline $\mathrm{O}(4)-\mathrm{C}(6)$ & $1.451(3)$ \\
\hline $\mathrm{O}(5)-\mathrm{C}(17)$ & $1.200(3)$ \\
\hline$C(1)-C(2)$ & $1.501(4)$ \\
\hline $\mathrm{C}(1)-\mathrm{C}(7)$ & $1.540(4)$ \\
\hline$C(2)-C(3)$ & $1.323(4)$ \\
\hline C(3)-C(4) & $1.495(4)$ \\
\hline$C(4)-C(5)$ & $1.531(3)$ \\
\hline$C(5)-C(6)$ & $1.501(4)$ \\
\hline C(7)-C(8) & $1.497(4)$ \\
\hline $\mathrm{C}(8)-\mathrm{C}(9)$ & $1.134(6)$ \\
\hline C(10)-C(15) & $1.391(4)$ \\
\hline C(10)-C(11) & 1.391(3) \\
\hline C(11)-C(12) & $1.389(5)$ \\
\hline C(12)-C(13) & $1.382(5)$ \\
\hline C(13)-C(14) & $1.399(3)$ \\
\hline C(14)-C(15) & $1.383(4)$ \\
\hline C(17)-C(18) & $1.489(3)$ \\
\hline C(18)-C(23) & $1.381(4)$ \\
\hline C(18)-C(19) & $1.402(4)$ \\
\hline C(19)-C(20) & $1.399(4)$ \\
\hline $\mathrm{C}(20)-\mathrm{C}(21)$ & $1.371(4)$ \\
\hline $\mathrm{C}(21)-\mathrm{C}(22)$ & $1.382(4)$ \\
\hline C(22)-C(23) & $1.400(4)$ \\
\hline $\mathrm{C}(5)-\mathrm{O}(1)-\mathrm{C}(1)$ & 113.72(19) \\
\hline $\mathrm{C}(15)-\mathrm{O}(2)-\mathrm{C}(4)$ & $119.4(2)$ \\
\hline
\end{tabular}




\begin{tabular}{|c|c|}
\hline $\mathrm{C}(12)-\mathrm{O}(3)-\mathrm{C}(16)$ & $116.0(3)$ \\
\hline $\mathrm{C}(17)-\mathrm{O}(4)-\mathrm{C}(6)$ & $116.4(2)$ \\
\hline $\mathrm{O}(1)-\mathrm{C}(1)-\mathrm{C}(2)$ & $111.2(2)$ \\
\hline $\mathrm{O}(1)-\mathrm{C}(1)-\mathrm{C}(7)$ & $111.4(2)$ \\
\hline$C(2)-C(1)-C(7)$ & $113.0(2)$ \\
\hline$C(3)-C(2)-C(1)$ & $122.0(3)$ \\
\hline$C(2)-C(3)-C(4)$ & $122.8(3)$ \\
\hline $\mathrm{O}(2)-\mathrm{C}(4)-\mathrm{C}(3)$ & $110.4(2)$ \\
\hline $\mathrm{O}(2)-\mathrm{C}(4)-\mathrm{C}(5)$ & $104.3(2)$ \\
\hline$C(3)-C(4)-C(5)$ & $109.4(2)$ \\
\hline $\mathrm{O}(1)-\mathrm{C}(5)-\mathrm{C}(6)$ & $107.6(2)$ \\
\hline $\mathrm{O}(1)-\mathrm{C}(5)-\mathrm{C}(4)$ & 109.2(3) \\
\hline$C(6)-C(5)-C(4)$ & 112.60(19) \\
\hline $\mathrm{O}(4)-\mathrm{C}(6)-\mathrm{C}(5)$ & $107.0(2)$ \\
\hline $\mathrm{C}(8)-\mathrm{C}(7)-\mathrm{C}(1)$ & 112.8(3) \\
\hline$C(9)-C(8)-C(7)$ & 133.1(5) \\
\hline $\mathrm{C}(15)-\mathrm{C}(10)-\mathrm{C}(11)$ & $119.0(3)$ \\
\hline $\mathrm{C}(12)-\mathrm{C}(11)-\mathrm{C}(10)$ & $121.0(3)$ \\
\hline $\mathrm{O}(3)-\mathrm{C}(12)-\mathrm{C}(13)$ & 124.1(3) \\
\hline $\mathrm{O}(3)-\mathrm{C}(12)-\mathrm{C}(11)$ & $116.0(3)$ \\
\hline $\mathrm{C}(13)-\mathrm{C}(12)-\mathrm{C}(11)$ & $119.8(2)$ \\
\hline $\mathrm{C}(12)-\mathrm{C}(13)-\mathrm{C}(14)$ & 119.4(3) \\
\hline $\mathrm{C}(15)-\mathrm{C}(14)-\mathrm{C}(13)$ & $120.5(2)$ \\
\hline $\mathrm{C}(14)-\mathrm{C}(15)-\mathrm{C}(10)$ & $120.2(2)$ \\
\hline $\mathrm{C}(14)-\mathrm{C}(15)-\mathrm{O}(2)$ & $114.7(2)$ \\
\hline $\mathrm{C}(10)-\mathrm{C}(15)-\mathrm{O}(2)$ & $125.1(2)$ \\
\hline $\mathrm{O}(5)-\mathrm{C}(17)-\mathrm{O}(4)$ & $124.0(3)$ \\
\hline $\mathrm{O}(5)-\mathrm{C}(17)-\mathrm{C}(18)$ & 124.3(3) \\
\hline $\mathrm{O}(4)-\mathrm{C}(17)-\mathrm{C}(18)$ & $111.7(2)$ \\
\hline C(23)-C(18)-C(19) & 119.7(3) \\
\hline $\mathrm{C}(23)-\mathrm{C}(18)-\mathrm{C}(17)$ & $118.5(2)$ \\
\hline $\mathrm{C}(19)-\mathrm{C}(18)-\mathrm{C}(17)$ & 121.8(3) \\
\hline C(20)-C(19)-C(18) & 119.8(3) \\
\hline $\mathrm{C}(21)-\mathrm{C}(20)-\mathrm{C}(19)$ & 119.1(3) \\
\hline $\mathrm{C}(20)-\mathrm{C}(21)-\mathrm{C}(22)$ & $122.5(3)$ \\
\hline $\mathrm{C}(20)-\mathrm{C}(21)-\mathrm{Br}(1)$ & $118.8(2)$ \\
\hline
\end{tabular}




$\begin{array}{ll}\mathrm{C}(22)-\mathrm{C}(21)-\operatorname{Br}(1) & 118.7(2) \\ \mathrm{C}(21)-\mathrm{C}(22)-\mathrm{C}(23) & 118.2(3) \\ \mathrm{C}(18)-\mathrm{C}(23)-\mathrm{C}(22) & 120.8(3)\end{array}$

Symmetry transformations used to generate equivalent atoms:

Table 4. Anisotropic displacement parameters $\left(\AA^{2} \times 10^{3}\right)$ for porco29. The anisotropic displacement factor exponent takes the form: $-2 \pi^{2}\left[h^{2} a^{* 2} U^{11}+\ldots+2 h k a^{*} b^{*} U^{12}\right]$

\begin{tabular}{|c|c|c|c|c|c|c|}
\hline & $\mathrm{U}^{11}$ & $\mathrm{U}^{22}$ & $\mathrm{U}^{33}$ & $\mathrm{U}^{23}$ & $\mathrm{U}^{13}$ & $\mathrm{U}^{12}$ \\
\hline $\operatorname{Br}(1)$ & $33(1)$ & $52(1)$ & $36(1)$ & $10(1)$ & $-4(1)$ & $4(1)$ \\
\hline $\mathrm{O}(1)$ & $22(1)$ & $31(1)$ & $27(1)$ & $-1(1)$ & $5(1)$ & $-7(1)$ \\
\hline $\mathrm{O}(2)$ & $26(1)$ & $24(1)$ & $36(1)$ & $7(1)$ & $-7(1)$ & $-2(1)$ \\
\hline $\mathrm{O}(3)$ & $29(1)$ & $42(1)$ & $32(1)$ & $8(1)$ & $-7(1)$ & $0(1)$ \\
\hline $\mathrm{O}(4)$ & $25(1)$ & $33(1)$ & $29(1)$ & $-3(1)$ & $-2(1)$ & $4(1)$ \\
\hline $\mathrm{O}(5)$ & $29(1)$ & $46(1)$ & $23(1)$ & $-3(1)$ & $2(1)$ & $3(1)$ \\
\hline $\mathrm{C}(1)$ & $27(1)$ & $30(2)$ & $28(1)$ & $-2(1)$ & $1(1)$ & $-2(1)$ \\
\hline$C(2)$ & $32(1)$ & $29(2)$ & $32(1)$ & $3(1)$ & $6(1)$ & $5(1)$ \\
\hline C(3) & $29(1)$ & $24(1)$ & $33(1)$ & $7(1)$ & $2(1)$ & $2(1)$ \\
\hline$C(4)$ & $23(1)$ & $25(1)$ & $27(1)$ & $3(1)$ & $-1(1)$ & $-2(1)$ \\
\hline C(5) & $22(1)$ & $25(1)$ & $25(1)$ & $3(2)$ & $1(1)$ & $-1(1)$ \\
\hline$C(6)$ & $27(1)$ & $35(2)$ & $26(1)$ & $0(1)$ & $-3(1)$ & $4(1)$ \\
\hline$C(7)$ & $30(1)$ & $43(2)$ & $29(2)$ & $6(1)$ & $6(1)$ & $0(1)$ \\
\hline$C(8)$ & $39(2)$ & $109(5)$ & $43(2)$ & $32(2)$ & $7(1)$ & $12(2)$ \\
\hline $\mathrm{C}(9)$ & $51(2)$ & $91(4)$ & $115(4)$ & $38(3)$ & $-13(3)$ & $-15(2)$ \\
\hline $\mathrm{C}(10)$ & $28(1)$ & $21(1)$ & $27(1)$ & $1(1)$ & $2(1)$ & $-2(1)$ \\
\hline $\mathrm{C}(11)$ & $33(1)$ & $24(2)$ & $23(1)$ & $5(1)$ & $0(1)$ & $5(1)$ \\
\hline $\mathrm{C}(12)$ & $25(1)$ & $28(1)$ & $22(1)$ & $-5(2)$ & $1(1)$ & $2(2)$ \\
\hline $\mathrm{C}(13)$ & $24(1)$ & $27(2)$ & $22(1)$ & $-2(1)$ & $3(1)$ & $-2(1)$ \\
\hline $\mathrm{C}(14)$ & $26(1)$ & $22(1)$ & $24(1)$ & $3(1)$ & $3(1)$ & $4(1)$ \\
\hline$C(15)$ & $22(1)$ & $23(2)$ & $22(1)$ & 1(1) & $-1(1)$ & $4(1)$ \\
\hline $\mathrm{C}(16)$ & $30(1)$ & $51(2)$ & $36(2)$ & 4(2) & $-5(1)$ & $-2(1)$ \\
\hline $\mathrm{C}(17)$ & $20(1)$ & $34(2)$ & $27(1)$ & $0(1)$ & $9(1)$ & $-6(1)$ \\
\hline$C(18)$ & $19(1)$ & $29(2)$ & $24(1)$ & $3(1)$ & $5(1)$ & $-4(1)$ \\
\hline $\mathrm{C}(19)$ & $29(1)$ & $35(1)$ & $28(1)$ & $-6(2)$ & $5(1)$ & $-2(2)$ \\
\hline
\end{tabular}




\begin{tabular}{lllllll}
$\mathrm{C}(20)$ & $29(1)$ & $41(2)$ & $23(1)$ & $-3(1)$ & $-1(1)$ & $-3(1)$ \\
$\mathrm{C}(21)$ & $20(1)$ & $38(2)$ & $26(1)$ & $7(1)$ & $2(1)$ & $-2(1)$ \\
$\mathrm{C}(22)$ & $26(1)$ & $33(2)$ & $32(1)$ & $0(1)$ & $6(1)$ & $5(1)$ \\
$\mathrm{C}(23)$ & $24(1)$ & $37(2)$ & $24(1)$ & $-3(1)$ & $3(1)$ & $-1(1)$ \\
\hline
\end{tabular}


Table 5. Hydrogen coordinates ( x $10^{4}$ ) and isotropic displacement parameters $\left(\AA^{2} \times 10^{3}\right)$ for porco29.

\begin{tabular}{|c|c|c|c|c|}
\hline & $\mathrm{x}$ & $\mathrm{y}$ & $\mathrm{z}$ & $\mathrm{U}(\mathrm{eq})$ \\
\hline $\mathrm{H}(1)$ & $-5020(30)$ & 17010(60) & 3393(14) & $22(7)$ \\
\hline $\mathrm{H}(2)$ & $-7360(20)$ & $17400(70)$ & $3401(12)$ & $15(6)$ \\
\hline $\mathrm{H}(3)$ & $-8530(40)$ & 16480(80) & 2332(19) & $58(12)$ \\
\hline $\mathrm{H}(4)$ & $-6930(30)$ & 14820(60) & $1573(14)$ & $19(7)$ \\
\hline $\mathrm{H}(5)$ & $-6170(30)$ & $11490(60)$ & 2741(15) & $24(8)$ \\
\hline $\mathrm{H}(6 \mathrm{~B})$ & $-4790(30)$ & $12500(90)$ & 1638(15) & $35(8)$ \\
\hline $\mathrm{H}(6 \mathrm{~A})$ & $-5680(30)$ & 10180(60) & $1684(14)$ & 19(7) \\
\hline $\mathrm{H}(7 \mathrm{~B})$ & $-5840(40)$ & $12860(110)$ & 3869(18) & $50(10)$ \\
\hline $\mathrm{H}(7 \mathrm{~A})$ & $-5640(30)$ & $14890(70)$ & 4336(16) & 33(9) \\
\hline $\mathrm{H}(8)$ & -3410 & 12923 & 3900 & 76 \\
\hline $\mathrm{H}(9 \mathrm{~B})$ & -3610 & 14031 & 5174 & 107 \\
\hline $\mathrm{H}(9 \mathrm{~A})$ & -2304 & 13056 & 4849 & 107 \\
\hline $\mathrm{H}(10)$ & $-8930(40)$ & $15600(80)$ & $911(17)$ & $46(10)$ \\
\hline $\mathrm{H}(11)$ & $-10890(30)$ & $15700(70)$ & $130(16)$ & 33(9) \\
\hline $\mathrm{H}(13)$ & $-12030(30)$ & $9340(60)$ & $829(14)$ & $16(7)$ \\
\hline $\mathrm{H}(14)$ & $-10090(30)$ & $8960(60)$ & 1596(15) & $27(8)$ \\
\hline $\mathrm{H}(16 \mathrm{C})$ & $-13490(30)$ & $9490(70)$ & $-219(16)$ & $29(8)$ \\
\hline $\mathrm{H}(16 \mathrm{~B})$ & $-14200(40)$ & 10850(80) & $330(20)$ & $57(12)$ \\
\hline $\mathrm{H}(16 \mathrm{~A})$ & $-14700(40)$ & 11440(80) & $-501(19)$ & $60(12)$ \\
\hline $\mathrm{H}(19)$ & $-2220(30)$ & 9390(70) & $3442(16)$ & $31(8)$ \\
\hline $\mathrm{H}(20)$ & $-740(30)$ & $7230(80)$ & $4223(15)$ & $44(9)$ \\
\hline $\mathrm{H}(22)$ & $-340(30)$ & $2130(80)$ & 2713(17) & $45(11)$ \\
\hline $\mathrm{H}(23)$ & $-1940(40)$ & $4140(90)$ & $2000(20)$ & 63(13) \\
\hline
\end{tabular}




\section{$\underline{\text { X-ray Crystal Structure of Compound 22: }}$}

Crystals of compound 22 suitable for x-ray analysis were obtained by slow evaporation from methylene chloride. Crystallographic data have been deposited with the Cambridge Crystallographic Data Centre (CCDC \# 287119). Copies of the data can be obtained free of charge on application to the CCDC, 12 Union Road, Cambridge CB21EZ, UK (fax: (+44)-1223-336-033; e-mail: deposit@ccdc.cam.ac.uk.

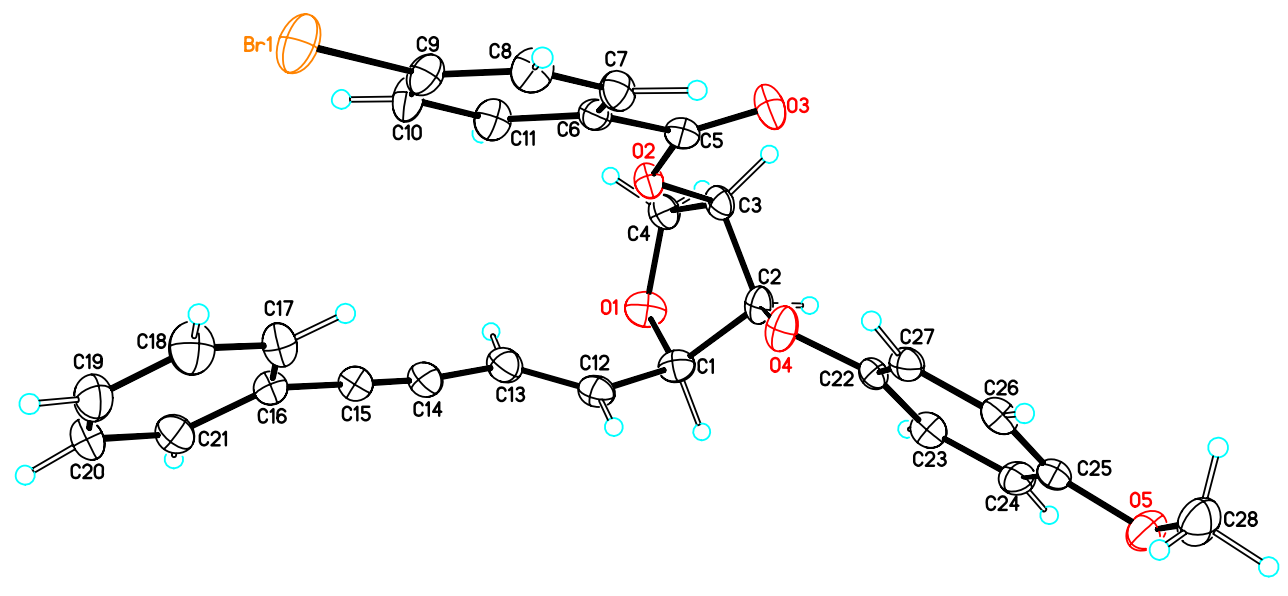

Table 1. Crystal data and structure refinement for porco40.

Identification code

Empirical formula

Formula weight

Temperature

Wavelength

Crystal system

Space group

Unit cell dimensions

Volume

Z

Density (calculated)

Absorption coefficient

$\mathrm{F}(000)$

Crystal size porco40

C28 H23 Br O5

519.37

173(2) K

$0.71073 \AA$

Orthorhombic

P2(1)2(1)2(1)

$\mathrm{a}=6.0107(3) \AA$

$\alpha=90^{\circ}$.

$\mathrm{b}=15.0074(7) \AA$

$\beta=90^{\circ}$

$c=25.3448(11) \AA$

$\gamma=90^{\circ}$.

2286.23(19) $\AA^{3}$

4

$1.509 \mathrm{Mg} / \mathrm{m}^{3}$

$1.837 \mathrm{~mm}^{-1}$

1064

$0.40 \times 0.10 \times 0.05 \mathrm{~mm}^{3}$ 
Theta range for data collection

Index ranges

Reflections collected

Independent reflections

Completeness to theta $=28.81^{\circ}$

Absorption correction

Max. and min. transmission

Refinement method

Data / restraints / parameters

Goodness-of-fit on $\mathrm{F}^{2}$

Final R indices [I $>2 \operatorname{sigma(I)]~}$

$\mathrm{R}$ indices (all data)

Absolute structure parameter

Largest diff. peak and hole
1.58 to $28.81^{\circ}$.

$-8<=\mathrm{h}<=7,-20<=\mathrm{k}<=20,-34<=\mathrm{l}<=26$

15307

5894 [R(int) $=0.0265]$

$99.4 \%$

Semiempirical by SADABS

0.9138 and 0.5270

Full-matrix least-squares on $\mathrm{F}^{2}$

5894 / 0 / 399

0.982

$\mathrm{R} 1=0.0333, \mathrm{wR} 2=0.0685$

$\mathrm{R} 1=0.0456, \mathrm{wR} 2=0.0727$

$0.000(6)$

0.760 and -0.418 e. $\AA^{-3}$

Table 2. Atomic coordinates $\left(\mathrm{x} 10^{4}\right)$ and equivalent isotropic displacement parameters $\left(\AA^{2} \mathrm{x} 10^{3}\right)$ for porco40. $\mathrm{U}(\mathrm{eq})$ is defined as one third of the trace of the orthogonalized $\mathrm{U}^{\mathrm{ij}}$ tensor.

\begin{tabular}{|c|c|c|c|c|}
\hline & $\mathrm{X}$ & $\mathrm{y}$ & $\mathrm{z}$ & $\mathrm{U}(\mathrm{eq})$ \\
\hline $\operatorname{Br}(1)$ & $5574(1)$ & 5493(1) & $1756(1)$ & $44(1)$ \\
\hline $\mathrm{O}(1)$ & $-4883(2)$ & $904(1)$ & 1707(1) & $35(1)$ \\
\hline $\mathrm{O}(2)$ & $-2036(2)$ & $2357(1)$ & 1129(1) & $24(1)$ \\
\hline $\mathrm{O}(3)$ & $-816(3)$ & 2601(1) & 299(1) & $34(1)$ \\
\hline $\mathrm{O}(4)$ & $-288(2)$ & $788(1)$ & $809(1)$ & $32(1)$ \\
\hline $\mathrm{O}(5)$ & $3564(3)$ & $-1708(1)$ & $-510(1)$ & $38(1)$ \\
\hline $\mathrm{C}(1)$ & $-2764(4)$ & $548(1)$ & $1578(1)$ & $29(1)$ \\
\hline $\mathrm{C}(2)$ & $-2520(3)$ & 771(1) & $983(1)$ & $26(1)$ \\
\hline $\mathrm{C}(3)$ & $-3591(3)$ & $1688(1)$ & $946(1)$ & $25(1)$ \\
\hline $\mathrm{C}(4)$ & $-5412(4)$ & $1629(1)$ & $1354(1)$ & $28(1)$ \\
\hline $\mathrm{C}(5)$ & $-741(4)$ & 2753(1) & $765(1)$ & $25(1)$ \\
\hline $\mathrm{C}(6)$ & $774(4)$ & $3412(1)$ & 1017(1) & $23(1)$ \\
\hline $\mathrm{C}(7)$ & $2636(4)$ & 3693(1) & $741(1)$ & $29(1)$ \\
\hline $\mathrm{C}(8)$ & $4072(4)$ & $4306(1)$ & $960(1)$ & $31(1)$ \\
\hline $\mathrm{C}(9)$ & $3618(4)$ & $4641(1)$ & $1457(1)$ & $30(1)$ \\
\hline $\mathrm{C}(10)$ & 1783(4) & $4373(1)$ & $1736(1)$ & $33(1)$ \\
\hline $\mathrm{C}(11)$ & $367(4)$ & $3745(1)$ & $1520(1)$ & $30(1)$ \\
\hline
\end{tabular}




\begin{tabular}{lrrrr}
$C(12)$ & $-912(4)$ & $927(1)$ & $1907(1)$ & $29(1)$ \\
$C(13)$ & $-1207(4)$ & $1501(2)$ & $2297(1)$ & $32(1)$ \\
$C(14)$ & $571(5)$ & $1937(1)$ & $2569(1)$ & $34(1)$ \\
$C(15)$ & $2007(4)$ & $2367(1)$ & $2775(1)$ & $33(1)$ \\
$C(16)$ & $3762(4)$ & $2882(1)$ & $3005(1)$ & $31(1)$ \\
$C(17)$ & $5234(4)$ & $3347(2)$ & $2689(1)$ & $37(1)$ \\
$C(18)$ & $6942(5)$ & $3840(2)$ & $2902(1)$ & $45(1)$ \\
$C(19)$ & $7215(5)$ & $3871(2)$ & $3440(1)$ & $41(1)$ \\
$C(20)$ & $5770(5)$ & $3426(2)$ & $3761(1)$ & $40(1)$ \\
$C(21)$ & $4045(5)$ & $2929(2)$ & $3551(1)$ & $36(1)$ \\
$C(22)$ & $505(4)$ & $154(1)$ & $461(1)$ & $23(1)$ \\
$C(23)$ & $-368(4)$ & $-700(1)$ & $403(1)$ & $29(1)$ \\
$C(24)$ & $694(4)$ & $-1296(1)$ & $66(1)$ & $30(1)$ \\
$C(25)$ & $2619(4)$ & $-1056(1)$ & $-200(1)$ & $27(1)$ \\
$C(26)$ & $3461(4)$ & $-208(1)$ & $-143(1)$ & $27(1)$ \\
$C(27)$ & $2384(3)$ & $395(1)$ & $187(1)$ & $25(1)$ \\
$C(28)$ & $5415(5)$ & $-1458(2)$ & $-827(1)$ & $43(1)$ \\
& & & & \\
\hline
\end{tabular}

Table 3. Bond lengths $[\AA]$ and angles $\left[{ }^{\circ}\right]$ for porco 40 .

\begin{tabular}{ll}
\hline $\mathrm{Br}(1)-\mathrm{C}(9)$ & $1.895(2)$ \\
$\mathrm{O}(1)-\mathrm{C}(1)$ & $1.419(3)$ \\
$\mathrm{O}(1)-\mathrm{C}(4)$ & $1.445(3)$ \\
$\mathrm{O}(2)-\mathrm{C}(5)$ & $1.346(2)$ \\
$\mathrm{O}(2)-\mathrm{C}(3)$ & $1.448(2)$ \\
$\mathrm{O}(3)-\mathrm{C}(5)$ & $1.203(2)$ \\
$\mathrm{O}(4)-\mathrm{C}(22)$ & $1.381(2)$ \\
$\mathrm{O}(4)-\mathrm{C}(2)$ & $1.414(3)$ \\
$\mathrm{O}(5)-\mathrm{C}(25)$ & $1.377(2)$ \\
$\mathrm{O}(5)-\mathrm{C}(28)$ & $1.422(3)$ \\
$\mathrm{C}(1)-\mathrm{C}(12)$ & $1.503(3)$ \\
$\mathrm{C}(1)-\mathrm{C}(2)$ & $1.550(3)$ \\
$\mathrm{C}(2)-\mathrm{C}(3)$ & $1.523(3)$ \\
$\mathrm{C}(3)-\mathrm{C}(4)$ & $1.508(3)$ \\
$\mathrm{C}(5)-\mathrm{C}(6)$ & $1.488(3)$ \\
$\mathrm{C}(6)-\mathrm{C}(7)$ & $1.386(3)$
\end{tabular}




\begin{tabular}{|c|c|}
\hline$C(6)-C(11)$ & $1.391(3)$ \\
\hline $\mathrm{C}(7)-\mathrm{C}(8)$ & $1.379(3)$ \\
\hline $\mathrm{C}(8)-\mathrm{C}(9)$ & $1.383(3)$ \\
\hline $\mathrm{C}(9)-\mathrm{C}(10)$ & $1.370(3)$ \\
\hline $\mathrm{C}(10)-\mathrm{C}(11)$ & $1.383(3)$ \\
\hline $\mathrm{C}(12)-\mathrm{C}(13)$ & $1.321(3)$ \\
\hline$C(13)-C(14)$ & $1.431(3)$ \\
\hline$C(14)-C(15)$ & $1.198(3)$ \\
\hline$C(15)-C(16)$ & $1.431(3)$ \\
\hline$C(16)-C(17)$ & $1.382(3)$ \\
\hline$C(16)-C(21)$ & $1.398(3)$ \\
\hline $\mathrm{C}(17)-\mathrm{C}(18)$ & $1.377(4)$ \\
\hline $\mathrm{C}(18)-\mathrm{C}(19)$ & $1.372(4)$ \\
\hline C(19)-C(20) & $1.365(4)$ \\
\hline$C(20)-C(21)$ & $1.384(4)$ \\
\hline$C(22)-C(27)$ & $1.375(3)$ \\
\hline$C(22)-C(23)$ & 1.392(3) \\
\hline C(23)-C(24) & $1.391(3)$ \\
\hline C(24)-C(25) & $1.387(3)$ \\
\hline$C(25)-C(26)$ & $1.378(3)$ \\
\hline $\mathrm{C}(26)-\mathrm{C}(27)$ & 1.391(3) \\
\hline $\mathrm{C}(1)-\mathrm{O}(1)-\mathrm{C}(4)$ & 109.72(16) \\
\hline $\mathrm{C}(5)-\mathrm{O}(2)-\mathrm{C}(3)$ & $117.45(15)$ \\
\hline $\mathrm{C}(22)-\mathrm{O}(4)-\mathrm{C}(2)$ & $121.00(15)$ \\
\hline $\mathrm{C}(25)-\mathrm{O}(5)-\mathrm{C}(28)$ & $117.20(18)$ \\
\hline $\mathrm{O}(1)-\mathrm{C}(1)-\mathrm{C}(12)$ & 113.20(17) \\
\hline $\mathrm{O}(1)-\mathrm{C}(1)-\mathrm{C}(2)$ & 103.23(17) \\
\hline $\mathrm{C}(12)-\mathrm{C}(1)-\mathrm{C}(2)$ & $112.87(17)$ \\
\hline $\mathrm{O}(4)-\mathrm{C}(2)-\mathrm{C}(3)$ & $111.44(17)$ \\
\hline $\mathrm{O}(4)-\mathrm{C}(2)-\mathrm{C}(1)$ & 113.48(17) \\
\hline $\mathrm{C}(3)-\mathrm{C}(2)-\mathrm{C}(1)$ & 102.38(17) \\
\hline $\mathrm{O}(2)-\mathrm{C}(3)-\mathrm{C}(4)$ & 106.86(16) \\
\hline $\mathrm{O}(2)-\mathrm{C}(3)-\mathrm{C}(2)$ & 109.54(16) \\
\hline$C(4)-C(3)-C(2)$ & $102.22(17)$ \\
\hline $\mathrm{O}(1)-\mathrm{C}(4)-\mathrm{C}(3)$ & $107.98(18)$ \\
\hline
\end{tabular}




\begin{tabular}{|c|c|}
\hline $\mathrm{O}(3)-\mathrm{C}(5)-\mathrm{O}(2)$ & $124.55(19)$ \\
\hline $\mathrm{O}(3)-\mathrm{C}(5)-\mathrm{C}(6)$ & 124.76(19) \\
\hline $\mathrm{O}(2)-\mathrm{C}(5)-\mathrm{C}(6)$ & $110.69(16)$ \\
\hline$C(7)-C(6)-C(11)$ & 119.75(19) \\
\hline$C(7)-C(6)-C(5)$ & 118.62(18) \\
\hline$C(11)-C(6)-C(5)$ & 121.63(19) \\
\hline$C(8)-C(7)-C(6)$ & $120.28(19)$ \\
\hline$C(7)-C(8)-C(9)$ & 119.1(2) \\
\hline$C(10)-C(9)-C(8)$ & $121.5(2)$ \\
\hline $\mathrm{C}(10)-\mathrm{C}(9)-\mathrm{Br}(1)$ & 119.39(16) \\
\hline $\mathrm{C}(8)-\mathrm{C}(9)-\mathrm{Br}(1)$ & 119.09(16) \\
\hline$C(9)-C(10)-C(11)$ & $119.4(2)$ \\
\hline$C(10)-C(11)-C(6)$ & $120.0(2)$ \\
\hline $\mathrm{C}(13)-\mathrm{C}(12)-\mathrm{C}(1)$ & $124.2(2)$ \\
\hline $\mathrm{C}(12)-\mathrm{C}(13)-\mathrm{C}(14)$ & $123.9(2)$ \\
\hline $\mathrm{C}(15)-\mathrm{C}(14)-\mathrm{C}(13)$ & $174.4(2)$ \\
\hline $\mathrm{C}(14)-\mathrm{C}(15)-\mathrm{C}(16)$ & $178.0(2)$ \\
\hline $\mathrm{C}(17)-\mathrm{C}(16)-\mathrm{C}(21)$ & $118.0(2)$ \\
\hline $\mathrm{C}(17)-\mathrm{C}(16)-\mathrm{C}(15)$ & $120.6(2)$ \\
\hline $\mathrm{C}(21)-\mathrm{C}(16)-\mathrm{C}(15)$ & $121.3(2)$ \\
\hline $\mathrm{C}(18)-\mathrm{C}(17)-\mathrm{C}(16)$ & $121.4(2)$ \\
\hline C(19)-C(18)-C(17) & 119.8(3) \\
\hline $\mathrm{C}(20)-\mathrm{C}(19)-\mathrm{C}(18)$ & $120.0(3)$ \\
\hline $\mathrm{C}(19)-\mathrm{C}(20)-\mathrm{C}(21)$ & $120.7(2)$ \\
\hline $\mathrm{C}(20)-\mathrm{C}(21)-\mathrm{C}(16)$ & $120.0(2)$ \\
\hline $\mathrm{C}(27)-\mathrm{C}(22)-\mathrm{O}(4)$ & $115.14(17)$ \\
\hline $\mathrm{C}(27)-\mathrm{C}(22)-\mathrm{C}(23)$ & 119.86(18) \\
\hline $\mathrm{O}(4)-\mathrm{C}(22)-\mathrm{C}(23)$ & 124.86(19) \\
\hline $\mathrm{C}(24)-\mathrm{C}(23)-\mathrm{C}(22)$ & $118.9(2)$ \\
\hline $\mathrm{C}(25)-\mathrm{C}(24)-\mathrm{C}(23)$ & 120.97(19) \\
\hline $\mathrm{O}(5)-\mathrm{C}(25)-\mathrm{C}(26)$ & $124.4(2)$ \\
\hline $\mathrm{O}(5)-\mathrm{C}(25)-\mathrm{C}(24)$ & 115.93(19) \\
\hline$C(26)-C(25)-C(24)$ & $119.6(2)$ \\
\hline $\mathrm{C}(25)-\mathrm{C}(26)-\mathrm{C}(27)$ & $119.6(2)$ \\
\hline$C(22)-C(27)-C(26)$ & 121.00(18) \\
\hline
\end{tabular}


Symmetry transformations used to generate equivalent atoms:

Table 4. Anisotropic displacement parameters $\left(\AA^{2} \times 10^{3}\right)$ for porco40. The anisotropic displacement factor exponent takes the form: $\quad-2 \pi^{2}\left[h^{2} a^{* 2} U^{11}+\ldots \quad+2 h k\right.$ a* $\left.b^{*} U^{12}\right]$

\begin{tabular}{|c|c|c|c|c|c|c|}
\hline & $\mathrm{U}^{11}$ & $\mathrm{U}^{22}$ & $\mathrm{U}^{33}$ & $\mathrm{U}^{23}$ & $\mathrm{U}^{13}$ & $\mathrm{U}^{12}$ \\
\hline $\operatorname{Br}(1)$ & $42(1)$ & $39(1)$ & $52(1)$ & $-14(1)$ & $9(1)$ & $-18(1)$ \\
\hline $\mathrm{O}(1)$ & $29(1)$ & $38(1)$ & $40(1)$ & $9(1)$ & $6(1)$ & $-3(1)$ \\
\hline $\mathrm{O}(2)$ & $26(1)$ & $28(1)$ & $20(1)$ & $-1(1)$ & $0(1)$ & $-6(1)$ \\
\hline $\mathrm{O}(3)$ & 39(1) & $45(1)$ & $19(1)$ & $-3(1)$ & $-1(1)$ & $-9(1)$ \\
\hline $\mathrm{O}(4)$ & 21(1) & $36(1)$ & $37(1)$ & $-14(1)$ & $0(1)$ & $-8(1)$ \\
\hline $\mathrm{O}(5)$ & $41(1)$ & $31(1)$ & $43(1)$ & $-5(1)$ & $2(1)$ & $7(1)$ \\
\hline $\mathrm{C}(1)$ & $26(1)$ & $26(1)$ & $36(1)$ & 2(1) & $0(1)$ & $-2(1)$ \\
\hline$C(2)$ & $20(1)$ & $29(1)$ & $28(1)$ & $-8(1)$ & $-4(1)$ & $-6(1)$ \\
\hline C(3) & 23(1) & $28(1)$ & $24(1)$ & $-2(1)$ & $-7(1)$ & $-6(1)$ \\
\hline $\mathrm{C}(4)$ & 23(1) & $33(1)$ & $28(1)$ & $-2(1)$ & $-4(1)$ & $-4(1)$ \\
\hline C(5) & $24(1)$ & $26(1)$ & $26(1)$ & $3(1)$ & $-2(1)$ & $2(1)$ \\
\hline$C(6)$ & $24(1)$ & $23(1)$ & $22(1)$ & $2(1)$ & $-2(1)$ & $0(1)$ \\
\hline $\mathrm{C}(7)$ & $30(1)$ & $33(1)$ & 23(1) & $-1(1)$ & $6(1)$ & $0(1)$ \\
\hline$C(8)$ & $28(1)$ & $33(1)$ & $33(1)$ & $1(1)$ & $13(1)$ & $-6(1)$ \\
\hline C(9) & $31(1)$ & $24(1)$ & $35(1)$ & $-4(1)$ & $2(1)$ & $-5(1)$ \\
\hline$C(10)$ & $37(1)$ & $33(1)$ & $28(1)$ & $-10(1)$ & $6(1)$ & $-7(1)$ \\
\hline$C(11)$ & $26(1)$ & $34(1)$ & $29(1)$ & $-1(1)$ & $9(1)$ & $-5(1)$ \\
\hline $\mathrm{C}(12)$ & $28(1)$ & $28(1)$ & $30(1)$ & $6(1)$ & $-2(1)$ & $-2(1)$ \\
\hline$C(13)$ & $35(1)$ & $33(1)$ & 29(1) & $3(1)$ & $-2(1)$ & $-1(1)$ \\
\hline$C(14)$ & $42(1)$ & $34(1)$ & $26(1)$ & $1(1)$ & $-2(1)$ & $6(1)$ \\
\hline$C(15)$ & $42(1)$ & $29(1)$ & $29(1)$ & $-1(1)$ & $-6(1)$ & $4(1)$ \\
\hline$C(16)$ & $38(1)$ & $27(1)$ & $29(1)$ & $-3(1)$ & $-7(1)$ & $7(1)$ \\
\hline $\mathrm{C}(17)$ & $46(2)$ & $39(1)$ & $26(1)$ & $-5(1)$ & $-6(1)$ & $0(1)$ \\
\hline$C(18)$ & $45(2)$ & $49(1)$ & $40(2)$ & $-3(1)$ & $5(1)$ & $-7(1)$ \\
\hline C(19) & $40(1)$ & $41(1)$ & $41(1)$ & $-9(1)$ & $-9(1)$ & $-1(1)$ \\
\hline$C(20)$ & $53(2)$ & $40(1)$ & $28(1)$ & $-5(1)$ & $-15(1)$ & $4(1)$ \\
\hline $\mathrm{C}(21)$ & $44(2)$ & $35(1)$ & $29(1)$ & $3(1)$ & $-1(1)$ & $1(1)$ \\
\hline $\mathrm{C}(22)$ & 23(1) & $26(1)$ & $20(1)$ & $-2(1)$ & $-6(1)$ & $1(1)$ \\
\hline C(23) & $25(1)$ & $32(1)$ & $29(1)$ & $3(1)$ & $-1(1)$ & $-4(1)$ \\
\hline
\end{tabular}




\begin{tabular}{lllllll}
$\mathrm{C}(24)$ & $35(1)$ & $21(1)$ & $33(1)$ & $2(1)$ & $-5(1)$ & $-4(1)$ \\
$\mathrm{C}(25)$ & $27(1)$ & $29(1)$ & $24(1)$ & $1(1)$ & $-8(1)$ & $6(1)$ \\
$\mathrm{C}(26)$ & $24(1)$ & $34(1)$ & $23(1)$ & $4(1)$ & $-2(1)$ & $-2(1)$ \\
$\mathrm{C}(27)$ & $25(1)$ & $24(1)$ & $27(1)$ & $1(1)$ & $-6(1)$ & $-3(1)$ \\
$\mathrm{C}(28)$ & $34(1)$ & $50(1)$ & $46(2)$ & $-10(1)$ & $3(1)$ & $10(1)$ \\
\hline
\end{tabular}

Table 5. Hydrogen coordinates ( $\times 10^{4}$ ) and isotropic displacement parameters $\left(\AA^{2} \times 10^{3}\right)$ for porco40.

\begin{tabular}{|c|c|c|c|c|}
\hline & $\mathrm{x}$ & $\mathrm{y}$ & $\mathrm{z}$ & $\mathrm{U}(\mathrm{eq})$ \\
\hline $\mathrm{H}(1)$ & $-2770(50)$ & $-151(18)$ & $1651(10)$ & $50(7)$ \\
\hline $\mathrm{H}(2)$ & $-3310(30)$ & 443(13) & 785(7) & $13(5)$ \\
\hline $\mathrm{H}(3)$ & $-4030(40)$ & 1839(12) & 594(8) & $17(5)$ \\
\hline $\mathrm{H}(4 \mathrm{~B})$ & $-5610(40)$ & 2162(14) & 1529(8) & $25(5)$ \\
\hline $\mathrm{H}(4 \mathrm{~A})$ & $-6770(40)$ & 1501(13) & 1178(8) & 16(5) \\
\hline $\mathrm{H}(7)$ & $2970(40)$ & $3428(14)$ & 379(9) & $31(6)$ \\
\hline $\mathrm{H}(8)$ & $5280(40)$ & $4471(14)$ & 807(9) & $29(6)$ \\
\hline $\mathrm{H}(10)$ & $1520(40)$ & $4611(14)$ & 2058(9) & $29(6)$ \\
\hline $\mathrm{H}(11)$ & $-830(40)$ & $3568(13)$ & 1698(8) & $20(5)$ \\
\hline $\mathrm{H}(12)$ & $500(40)$ & 749(14) & 1822(9) & $29(6)$ \\
\hline $\mathrm{H}(13)$ & $-2780(50)$ & $1650(16)$ & $2390(10)$ & $42(7)$ \\
\hline $\mathrm{H}(17)$ & 4990(50) & $3307(16)$ & 2336(11) & $45(7)$ \\
\hline $\mathrm{H}(18)$ & $7860(60)$ & 4156(19) & 2682(12) & 63(9) \\
\hline $\mathrm{H}(19)$ & $8410(60)$ & 4189(19) & $3585(11)$ & $62(9)$ \\
\hline $\mathrm{H}(20)$ & $5890(50)$ & $3405(15)$ & $4138(10)$ & $40(7)$ \\
\hline $\mathrm{H}(21)$ & $3120(50)$ & 2621(16) & $3740(10)$ & $42(8)$ \\
\hline $\mathrm{H}(23)$ & $-1730(40)$ & $-831(14)$ & $575(9)$ & $30(6)$ \\
\hline $\mathrm{H}(24)$ & $90(40)$ & $-1864(13)$ & $42(7)$ & $23(5)$ \\
\hline $\mathrm{H}(26)$ & $4720(40)$ & $-65(14)$ & $-314(9)$ & $24(6)$ \\
\hline $\mathrm{H}(27)$ & $2900(40)$ & 964(15) & 207(8) & $31(6)$ \\
\hline $\mathrm{H}(28 \mathrm{C})$ & $5060(50)$ & $-990(19)$ & $-1067(11)$ & $55(8)$ \\
\hline $\mathrm{H}(28 \mathrm{~B})$ & $6570(50)$ & $-1314(17)$ & $-639(11)$ & $47(8)$ \\
\hline $\mathrm{H}(28 \mathrm{~A})$ & $5890(70)$ & $-1980(20)$ & $-1033(13)$ & $84(11)$ \\
\hline
\end{tabular}




\section{X-ray Crystal Structure of Compound 24:}

Crystals of compound 24 suitable for x-ray analysis were obtained by slow evaporation from methylene chloride. Crystallographic data have been deposited with the Cambridge Crystallographic Data Centre (CCDC \# 287120). Copies of the data can be obtained free of charge on application to the CCDC, 12 Union Road, Cambridge CB21EZ, UK (fax: (+44)-1223-336-033; e-mail: deposit@ccdc.cam.ac.uk.

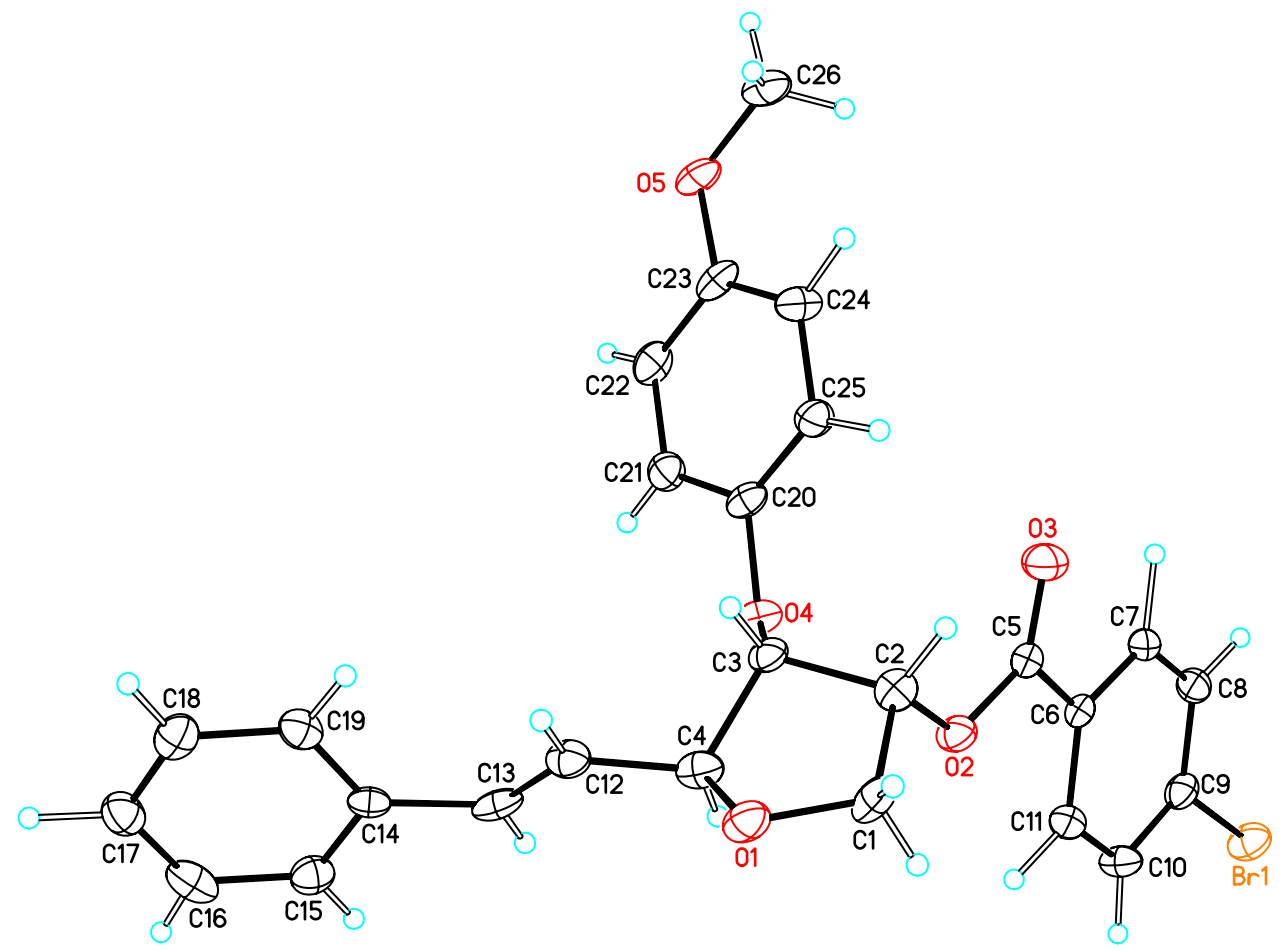

Table 1. Crystal data and structure refinement for porco41.

Identification code

Empirical formula

Formula weight

Temperature

Wavelength

Crystal system

Space group

Unit cell dimensions porco41

C26 H23 Br O5

495.35

173(2) K

$0.71073 \AA$

Monoclinic

P2(1)
$\mathrm{a}=13.1894(18) \AA$
$\alpha=90^{\circ}$.
$\mathrm{b}=5.1990(10) \AA$
$\beta=110.015(7)^{\circ}$. 
Volume

Z

Density (calculated)

Absorption coefficient

$\mathrm{F}(000)$

Crystal size

Theta range for data collection

Index ranges

Reflections collected

Independent reflections

Completeness to theta $=23.25^{\circ}$

Absorption correction

Max. and min. transmission

Refinement method

Data / restraints / parameters

Goodness-of-fit on $\mathrm{F}^{2}$

Final R indices [I $>2 \operatorname{sigma(I)]~}$

$\mathrm{R}$ indices (all data)

Absolute structure parameter

Largest diff. peak and hole $\mathrm{c}=17.152(3) \AA \quad \gamma=90^{\circ}$.

1105.1(3) $\AA^{3}$

2

$1.489 \mathrm{Mg} / \mathrm{m}^{3}$

$1.896 \mathrm{~mm}^{-1}$

508

$1.00 \times 0.10 \times 0.02 \mathrm{~mm}^{3}$

2.39 to $23.25^{\circ}$.

$-13<=\mathrm{h}<=14,-5<=\mathrm{k}<=5,-18<=\mathrm{l}<=19$

8090

3034 [R(int) $=0.0515]$

$97.5 \%$

Semiempirical by SADABS

0.9631 and 0.2529

Full-matrix least-squares on $\mathrm{F}^{2}$

3034 / 1 / 370

0.979

$\mathrm{R} 1=0.0388, \mathrm{wR} 2=0.0816$

$\mathrm{R} 1=0.0514, \mathrm{wR} 2=0.0857$

0.021(11)

0.872 and -0.401 e. $\AA^{-3}$

Table 2. Atomic coordinates $\left(\times 10^{4}\right)$ and equivalent isotropic displacement parameters $\left(\AA^{2} \times 10^{3}\right)$ for porco41. $\mathrm{U}(\mathrm{eq})$ is defined as one third of the trace of the orthogonalized $\mathrm{U}^{\mathrm{ij}}$ tensor.

\begin{tabular}{lrrrr}
\hline & $\mathrm{x}$ & $\mathrm{y}$ & $\mathrm{z}$ & $\mathrm{U}(\mathrm{eq})$ \\
\hline $\mathrm{Br}(1)$ & $1976(1)$ & $625(1)$ & $6242(1)$ & $36(1)$ \\
$\mathrm{O}(1)$ & $-1072(3)$ & $11408(6)$ & $9596(2)$ & $32(1)$ \\
$\mathrm{O}(2)$ & $-656(3)$ & $9035(6)$ & $7929(2)$ & $27(1)$ \\
$\mathrm{O}(3)$ & $-1476(3)$ & $10027(7)$ & $6580(2)$ & $39(1)$ \\
$\mathrm{O}(4)$ & $-2648(3)$ & $7511(6)$ & $7904(2)$ & $29(1)$ \\
$\mathrm{O}(5)$ & $-6583(3)$ & $7718(7)$ & $5321(2)$ & $34(1)$ \\
$\mathrm{C}(1)$ & $-583(4)$ & $12058(12)$ & $9005(4)$ & $34(1)$ \\
$\mathrm{C}(2)$ & $-1274(4)$ & $11035(11)$ & $8161(3)$ & $26(1)$ \\
$\mathrm{C}(3)$ & $-2228(4)$ & $9778(10)$ & $8353(3)$ & $25(1)$ \\
$\mathrm{C}(4)$ & $-1734(4)$ & $9168(11)$ & $9272(4)$ & $29(1)$
\end{tabular}




\begin{tabular}{lrlll}
$\mathrm{C}(5)$ & $-838(4)$ & $8770(10)$ & $7119(3)$ & $24(1)$ \\
$\mathrm{C}(6)$ & $-166(4)$ & $6698(9)$ & $6940(3)$ & $23(1)$ \\
$\mathrm{C}(7)$ & $-326(4)$ & $6164(10)$ & $6122(3)$ & $24(1)$ \\
$\mathrm{C}(8)$ & $295(4)$ & $4299(11)$ & $5906(4)$ & $30(1)$ \\
$\mathrm{C}(9)$ & $1094(4)$ & $3087(10)$ & $6535(3)$ & $26(1)$ \\
$\mathrm{C}(10)$ & $1287(4)$ & $3566(11)$ & $7363(4)$ & $29(1)$ \\
$\mathrm{C}(11)$ & $641(3)$ & $5411(14)$ & $7567(3)$ & $28(1)$ \\
$\mathrm{C}(12)$ & $-2502(4)$ & $8835(11)$ & $9729(3)$ & $28(1)$ \\
$\mathrm{C}(13)$ & $-2597(4)$ & $6705(12)$ & $10117(3)$ & $30(1)$ \\
$\mathrm{C}(14)$ & $-3300(4)$ & $6256(8)$ & $10610(3)$ & $23(1)$ \\
$\mathrm{C}(15)$ & $-3120(4)$ & $4139(11)$ & $11136(4)$ & $31(1)$ \\
$\mathrm{C}(16)$ & $-3717(5)$ & $3796(13)$ & $11661(4)$ & $38(2)$ \\
$\mathrm{C}(17)$ & $-4507(4)$ & $5549(16)$ & $11653(3)$ & $34(1)$ \\
$\mathrm{C}(18)$ & $-4705(4)$ & $7609(11)$ & $11124(4)$ & $32(1)$ \\
$\mathrm{C}(19)$ & $-4120(4)$ & $7982(11)$ & $10598(3)$ & $28(1)$ \\
$\mathrm{C}(20)$ & $-3636(4)$ & $7744(9)$ & $7239(3)$ & $25(1)$ \\
$\mathrm{C}(21)$ & $-4409(4)$ & $5838(14)$ & $7188(3)$ & $30(1)$ \\
$\mathrm{C}(22)$ & $-5386(4)$ & $5920(14)$ & $6545(3)$ & $31(1)$ \\
$\mathrm{C}(23)$ & $-5591(4)$ & $7839(10)$ & $5947(3)$ & $29(1)$ \\
$\mathrm{C}(24)$ & $-4830(4)$ & $9709(10)$ & $5995(4)$ & $32(2)$ \\
$\mathrm{C}(25)$ & $-3833(4)$ & $9635(10)$ & $6652(3)$ & $30(1)$ \\
$\mathrm{C}(26)$ & $-6799(4)$ & $9614(11)$ & $4689(3)$ & $36(2)$ \\
\hline
\end{tabular}

Table 3. Bond lengths $[\AA]$ and angles $\left[{ }^{\circ}\right]$ for porco41.

\begin{tabular}{ll}
\hline $\mathrm{Br}(1)-\mathrm{C}(9)$ & $1.909(5)$ \\
$\mathrm{O}(1)-\mathrm{C}(1)$ & $1.417(7)$ \\
$\mathrm{O}(1)-\mathrm{C}(4)$ & $1.448(6)$ \\
$\mathrm{O}(2)-\mathrm{C}(5)$ & $1.334(6)$ \\
$\mathrm{O}(2)-\mathrm{C}(2)$ & $1.457(6)$ \\
$\mathrm{O}(3)-\mathrm{C}(5)$ & $1.208(6)$ \\
$\mathrm{O}(4)-\mathrm{C}(20)$ & $1.412(6)$ \\
$\mathrm{O}(4)-\mathrm{C}(3)$ & $1.413(6)$ \\
$\mathrm{O}(5)-\mathrm{C}(23)$ & $1.381(6)$ \\
$\mathrm{O}(5)-\mathrm{C}(26)$ & $1.420(6)$ \\
$\mathrm{C}(1)-\mathrm{C}(2)$ & $1.519(7)$
\end{tabular}




\begin{tabular}{|c|c|}
\hline $\mathrm{C}(2)-\mathrm{C}(3)$ & $1.551(7)$ \\
\hline$C(3)-C(4)$ & $1.520(8)$ \\
\hline$C(4)-C(12)$ & $1.488(7)$ \\
\hline$C(5)-C(6)$ & $1.492(7)$ \\
\hline$C(6)-C(7)$ & $1.374(6)$ \\
\hline$C(6)-C(11)$ & $1.398(7)$ \\
\hline $\mathrm{C}(7)-\mathrm{C}(8)$ & $1.398(7)$ \\
\hline $\mathrm{C}(8)-\mathrm{C}(9)$ & $1.376(7)$ \\
\hline $\mathrm{C}(9)-\mathrm{C}(10)$ & $1.377(7)$ \\
\hline $\mathrm{C}(10)-\mathrm{C}(11)$ & $1.404(8)$ \\
\hline $\mathrm{C}(12)-\mathrm{C}(13)$ & $1.319(8)$ \\
\hline C(13)-C(14) & $1.472(7)$ \\
\hline C(14)-C(15) & $1.392(7)$ \\
\hline C(14)-C(19) & $1.401(7)$ \\
\hline C(15)-C(16) & $1.395(8)$ \\
\hline $\mathrm{C}(16)-\mathrm{C}(17)$ & $1.381(9)$ \\
\hline C(17)-C(18) & $1.371(9)$ \\
\hline C(18)-C(19) & $1.386(7)$ \\
\hline C(20)-C(25) & $1.367(7)$ \\
\hline$C(20)-C(21)$ & $1.403(8)$ \\
\hline$C(21)-C(22)$ & $1.381(7)$ \\
\hline C(22)-C(23) & $1.390(8)$ \\
\hline C(23)-C(24) & $1.380(7)$ \\
\hline C(24)-C(25) & $1.409(8)$ \\
\hline $\mathrm{C}(1)-\mathrm{O}(1)-\mathrm{C}(4)$ & 106.2(4) \\
\hline $\mathrm{C}(5)-\mathrm{O}(2)-\mathrm{C}(2)$ & 116.1(4) \\
\hline $\mathrm{C}(20)-\mathrm{O}(4)-\mathrm{C}(3)$ & 116.7(4) \\
\hline $\mathrm{C}(23)-\mathrm{O}(5)-\mathrm{C}(26)$ & 116.9(4) \\
\hline $\mathrm{O}(1)-\mathrm{C}(1)-\mathrm{C}(2)$ & 109.1(4) \\
\hline $\mathrm{O}(2)-\mathrm{C}(2)-\mathrm{C}(1)$ & 107.5(4) \\
\hline $\mathrm{O}(2)-\mathrm{C}(2)-\mathrm{C}(3)$ & 109.1(4) \\
\hline $\mathrm{C}(1)-\mathrm{C}(2)-\mathrm{C}(3)$ & 102.2(4) \\
\hline $\mathrm{O}(4)-\mathrm{C}(3)-\mathrm{C}(4)$ & 109.9(4) \\
\hline $\mathrm{O}(4)-\mathrm{C}(3)-\mathrm{C}(2)$ & 115.5(4) \\
\hline$C(4)-C(3)-C(2)$ & $102.6(4)$ \\
\hline
\end{tabular}




\begin{tabular}{|c|c|}
\hline $\mathrm{O}(1)-\mathrm{C}(4)-\mathrm{C}(12)$ & $108.7(4)$ \\
\hline $\mathrm{O}(1)-\mathrm{C}(4)-\mathrm{C}(3)$ & $102.6(4)$ \\
\hline$C(12)-C(4)-C(3)$ & $116.3(4)$ \\
\hline $\mathrm{O}(3)-\mathrm{C}(5)-\mathrm{O}(2)$ & $124.9(5)$ \\
\hline $\mathrm{O}(3)-\mathrm{C}(5)-\mathrm{C}(6)$ & $122.7(5)$ \\
\hline $\mathrm{O}(2)-\mathrm{C}(5)-\mathrm{C}(6)$ & $112.4(4)$ \\
\hline$C(7)-C(6)-C(11)$ & $120.0(5)$ \\
\hline$C(7)-C(6)-C(5)$ & $117.5(4)$ \\
\hline$C(11)-C(6)-C(5)$ & $122.4(5)$ \\
\hline$C(6)-C(7)-C(8)$ & $120.8(5)$ \\
\hline$C(9)-C(8)-C(7)$ & 118.1(5) \\
\hline$C(8)-C(9)-C(10)$ & $123.2(5)$ \\
\hline $\mathrm{C}(8)-\mathrm{C}(9)-\mathrm{Br}(1)$ & 118.2(4) \\
\hline $\mathrm{C}(10)-\mathrm{C}(9)-\mathrm{Br}(1)$ & $118.6(4)$ \\
\hline $\mathrm{C}(9)-\mathrm{C}(10)-\mathrm{C}(11)$ & $117.8(5)$ \\
\hline$C(6)-C(11)-C(10)$ & 120.1(5) \\
\hline $\mathrm{C}(13)-\mathrm{C}(12)-\mathrm{C}(4)$ & $124.2(5)$ \\
\hline $\mathrm{C}(12)-\mathrm{C}(13)-\mathrm{C}(14)$ & $127.5(5)$ \\
\hline $\mathrm{C}(15)-\mathrm{C}(14)-\mathrm{C}(19)$ & $118.4(5)$ \\
\hline $\mathrm{C}(15)-\mathrm{C}(14)-\mathrm{C}(13)$ & $119.8(5)$ \\
\hline $\mathrm{C}(19)-\mathrm{C}(14)-\mathrm{C}(13)$ & 121.7(5) \\
\hline $\mathrm{C}(14)-\mathrm{C}(15)-\mathrm{C}(16)$ & $120.8(6)$ \\
\hline $\mathrm{C}(17)-\mathrm{C}(16)-\mathrm{C}(15)$ & $120.0(6)$ \\
\hline $\mathrm{C}(16)-\mathrm{C}(17)-\mathrm{C}(18)$ & 119.6(5) \\
\hline $\mathrm{C}(17)-\mathrm{C}(18)-\mathrm{C}(19)$ & 121.2(5) \\
\hline $\mathrm{C}(18)-\mathrm{C}(19)-\mathrm{C}(14)$ & $120.0(5)$ \\
\hline$C(25)-C(20)-C(21)$ & $120.7(5)$ \\
\hline $\mathrm{C}(25)-\mathrm{C}(20)-\mathrm{O}(4)$ & $123.2(5)$ \\
\hline $\mathrm{C}(21)-\mathrm{C}(20)-\mathrm{O}(4)$ & $116.0(5)$ \\
\hline $\mathrm{C}(22)-\mathrm{C}(21)-\mathrm{C}(20)$ & 119.2(6) \\
\hline$C(21)-C(22)-C(23)$ & 120.3(6) \\
\hline $\mathrm{C}(24)-\mathrm{C}(23)-\mathrm{O}(5)$ & 123.7(5) \\
\hline$C(24)-C(23)-C(22)$ & $120.5(5)$ \\
\hline $\mathrm{O}(5)-\mathrm{C}(23)-\mathrm{C}(22)$ & 115.8(5) \\
\hline$C(23)-C(24)-C(25)$ & 119.3(5) \\
\hline $\mathrm{C}(20)-\mathrm{C}(25)-\mathrm{C}(24)$ & $120.0(5)$ \\
\hline
\end{tabular}


Symmetry transformations used to generate equivalent atoms:

Table 4. Anisotropic displacement parameters $\left(\AA^{2} \times 10^{3}\right)$ for porco41. The anisotropic displacement factor exponent takes the form: $\quad-2 \pi^{2}\left[h^{2} a^{* 2} U^{11}+\ldots \quad+2 h \mathrm{k} \mathrm{a}^{*} \mathrm{~b}^{*} \mathrm{U}^{12}\right]$

\begin{tabular}{|c|c|c|c|c|c|c|}
\hline & $\mathrm{U}^{11}$ & $\mathrm{U}^{22}$ & $\mathrm{U}^{33}$ & $\mathrm{U}^{23}$ & $\mathrm{U}^{13}$ & $\mathrm{U}^{12}$ \\
\hline $\operatorname{Br}(1)$ & $28(1)$ & $40(1)$ & $40(1)$ & $-5(1)$ & $10(1)$ & $7(1)$ \\
\hline $\mathrm{O}(1)$ & $24(2)$ & $38(3)$ & $31(2)$ & $-9(2)$ & $5(2)$ & $-2(2)$ \\
\hline $\mathrm{O}(2)$ & $22(2)$ & $33(2)$ & $26(2)$ & $-2(2)$ & $8(2)$ & $6(2)$ \\
\hline $\mathrm{O}(3)$ & $31(2)$ & $52(3)$ & $31(2)$ & $8(2)$ & $9(2)$ & $13(2)$ \\
\hline $\mathrm{O}(4)$ & $23(2)$ & $26(2)$ & $31(2)$ & $-2(2)$ & $2(2)$ & $2(2)$ \\
\hline $\mathrm{O}(5)$ & $15(2)$ & $43(2)$ & $38(2)$ & $-6(2)$ & $1(2)$ & $-4(2)$ \\
\hline $\mathrm{C}(1)$ & $20(3)$ & $43(4)$ & $42(4)$ & $-15(3)$ & $13(3)$ & $-2(3)$ \\
\hline$C(2)$ & $29(3)$ & $19(4)$ & $32(3)$ & $-3(3)$ & $11(2)$ & 3(3) \\
\hline C(3) & 19(3) & $31(3)$ & $25(3)$ & $-2(2)$ & $7(2)$ & $7(2)$ \\
\hline$C(4)$ & $27(3)$ & $29(3)$ & $27(4)$ & $-6(3)$ & $4(3)$ & $5(3)$ \\
\hline C(5) & 19(3) & $30(3)$ & 23(3) & 2(3) & $8(3)$ & $-1(2)$ \\
\hline$C(6)$ & $16(2)$ & $29(3)$ & $24(3)$ & 2(2) & $7(2)$ & $-3(2)$ \\
\hline$C(7)$ & $21(2)$ & $29(4)$ & $22(3)$ & $5(3)$ & $8(2)$ & $3(2)$ \\
\hline C(8) & $27(3)$ & $43(3)$ & $18(4)$ & $-3(3)$ & $7(3)$ & $-5(3)$ \\
\hline C(9) & 21(3) & $27(3)$ & $33(4)$ & $0(3)$ & $12(3)$ & $3(2)$ \\
\hline$C(10)$ & $25(3)$ & $32(3)$ & $26(4)$ & $-1(3)$ & $2(3)$ & 6(3) \\
\hline $\mathrm{C}(11)$ & $25(2)$ & $37(3)$ & $21(3)$ & $0(4)$ & $6(2)$ & $-1(3)$ \\
\hline $\mathrm{C}(12)$ & $27(3)$ & $28(4)$ & $27(3)$ & $-3(3)$ & $7(3)$ & 8(3) \\
\hline $\mathrm{C}(13)$ & 18(3) & $27(3)$ & $36(4)$ & $-4(3)$ & $-1(3)$ & 8(3) \\
\hline$C(14)$ & $21(2)$ & $22(4)$ & 18(3) & $-5(2)$ & $-2(2)$ & $-4(2)$ \\
\hline$C(15)$ & $26(3)$ & 29(3) & $33(4)$ & $-1(3)$ & $6(3)$ & $-4(3)$ \\
\hline $\mathrm{C}(16)$ & $51(4)$ & $32(4)$ & $25(4)$ & 4(3) & $5(3)$ & $-5(3)$ \\
\hline $\mathrm{C}(17)$ & $36(3)$ & $35(3)$ & $30(3)$ & $3(4)$ & $11(2)$ & $-5(4)$ \\
\hline $\mathrm{C}(18)$ & 28(3) & $32(3)$ & $35(4)$ & $-10(3)$ & $10(3)$ & $-6(3)$ \\
\hline C(19) & $32(3)$ & $23(3)$ & $27(3)$ & 3(3) & $7(3)$ & $-4(2)$ \\
\hline$C(20)$ & 19(3) & $24(3)$ & $29(3)$ & $-8(3)$ & $6(3)$ & $2(2)$ \\
\hline $\mathrm{C}(21)$ & $30(3)$ & $30(3)$ & $31(3)$ & 2(4) & $14(3)$ & $5(3)$ \\
\hline $\mathrm{C}(22)$ & $25(3)$ & $26(4)$ & $42(3)$ & $-4(3)$ & $12(3)$ & $-11(3)$ \\
\hline C(23) & 16(3) & $30(3)$ & $40(4)$ & $-11(3)$ & $8(3)$ & $-2(2)$ \\
\hline
\end{tabular}




$\begin{array}{lllllll}\mathrm{C}(24) & 27(3) & 30(3) & 32(4) & 3(3) & 1(3) & 2(2) \\ \mathrm{C}(25) & 23(3) & 32(3) & 31(3) & 5(2) & 6(3) & -5(2) \\ \mathrm{C}(26) & 25(3) & 44(4) & 33(4) & -5(3) & 0(3) & 5(2)\end{array}$

Table 5. Hydrogen coordinates ( $\left.\times 10^{4}\right)$ and isotropic displacement parameters $\left(\AA^{2} \times 10^{3}\right)$ for porco41.

\begin{tabular}{|c|c|c|c|c|}
\hline & $\mathrm{x}$ & $\mathrm{y}$ & $\mathrm{z}$ & $\mathrm{U}(\mathrm{eq})$ \\
\hline $\mathrm{H}(26 \mathrm{~A})$ & -6837 & 11311 & 4926 & 54 \\
\hline $\mathrm{H}(26 \mathrm{~B})$ & -7489 & 9232 & 4253 & 54 \\
\hline $\mathrm{H}(26 \mathrm{C})$ & -6221 & 9607 & 4451 & 54 \\
\hline $\mathrm{H}(11)$ & $780(30)$ & $5420(120)$ & $8190(30)$ & 21(11) \\
\hline $\mathrm{H}(12)$ & $-2860(40)$ & 10580(150) & $9780(30)$ & $43(13)$ \\
\hline $\mathrm{H}(17)$ & $-4950(40)$ & $5520(150)$ & $12060(30)$ & $54(14)$ \\
\hline $\mathrm{H}(24)$ & $-5010(40)$ & $11220(100)$ & $5590(30)$ & $30(15)$ \\
\hline $\mathrm{H}(2)$ & $-1450(30)$ & 12360(90) & $7740(30)$ & $11(12)$ \\
\hline $\mathrm{H}(8)$ & $180(30)$ & $4170(80)$ & $5320(30)$ & $17(13)$ \\
\hline $\mathrm{H}(22)$ & $-5810(40)$ & $4690(100)$ & $6530(30)$ & $27(16)$ \\
\hline $\mathrm{H}(21)$ & $-4260(40)$ & $4710(100)$ & $7580(30)$ & $26(16)$ \\
\hline $\mathrm{H}(10)$ & $1860(40)$ & $2870(100)$ & $7780(30)$ & $36(16)$ \\
\hline $\mathrm{H}(15)$ & $-2600(40)$ & $2950(100)$ & $11140(30)$ & $28(15)$ \\
\hline $\mathrm{H}(13)$ & $-2200(30)$ & $5490(100)$ & 10130(20) & $1(11)$ \\
\hline $\mathrm{H}(7)$ & $-990(40)$ & $7140(100)$ & $5600(30)$ & $42(15)$ \\
\hline $\mathrm{H}(3)$ & $-2740(40)$ & 10890(120) & $8360(30)$ & $27(14)$ \\
\hline $\mathrm{H}(19)$ & $-4230(40)$ & $9330(90)$ & 10210(30) & $32(15)$ \\
\hline $\mathrm{H}(4)$ & $-1320(30)$ & $7680(90)$ & $9330(20)$ & $3(11)$ \\
\hline $\mathrm{H}(25)$ & $-3340(40)$ & 10900(130) & $6610(30)$ & $35(14)$ \\
\hline $\mathrm{H}(18)$ & $-5250(40)$ & $9010(100)$ & 11110(30) & $30(14)$ \\
\hline $\mathrm{H}(1 \mathrm{~B})$ & $100(40)$ & 11230(90) & $9130(30)$ & $24(13)$ \\
\hline $\mathrm{H}(1 \mathrm{~A})$ & $-570(40)$ & $14020(120)$ & $9070(30)$ & $53(18)$ \\
\hline $\mathrm{H}(16)$ & $-3540(40)$ & $2580(110)$ & $12020(30)$ & $42(18)$ \\
\hline
\end{tabular}

